Portland State University

PDXScholar

8-1-2017

\title{
Laser as a Tool to Study Radiation Effects in CMOS
}

Bahar Ajdari

Portland State University

Follow this and additional works at: https://pdxscholar.library.pdx.edu/open_access_etds

Part of the VLSI and Circuits, Embedded and Hardware Systems Commons Let us know how access to this document benefits you.

\section{Recommended Citation}

Ajdari, Bahar, "Laser as a Tool to Study Radiation Effects in CMOS" (2017). Dissertations and Theses. Paper 3769.

https://doi.org/10.15760/etd.5653

This Dissertation is brought to you for free and open access. It has been accepted for inclusion in Dissertations and Theses by an authorized administrator of PDXScholar. Please contact us if we can make this document more accessible: pdxscholar@pdx.edu. 
Laser as a Tool to Study Radiation Effects in CMOS

by

Bahar Ajdari

A dissertation submitted in partial fulfillment of the requirements for the degree of

Doctor of Philosophy

in

Applied Physics

\author{
Dissertation Committee: \\ Erik Bodegom, Chair \\ Ralf Widenhorn \\ Ricardo Ascazubi \\ Raj Solanki \\ Branimir Pejcinovic
}

Portland State University

2017 
(C) 2017 Bahar Ajdari 


\begin{abstract}
Energetic particles from cosmic ray or terrestrial sources can strike sensitive areas of CMOS devices and cause soft errors. Understanding the effects of such interactions is crucial as the device technology advances, and chip reliability has become more important than ever. Particle accelerator testing has been the standard method to characterize the sensitivity of chips to single event upsets (SEUs). However, because of their costs and availability limitations, other techniques have been explored. Pulsed laser has been a successful tool for characterization of SEU behavior, but to this day, laser has not been recognized as a comparable method to beam testing. In this thesis, I propose a methodology of correlating laser soft error rate (SER) to particle beam gathered data. Additionally, results are presented showing a temperature dependence of SER and the "neighbor effect" phenomenon where due to the close proximity of devices a "weakening effect" in the ON state can be observed.
\end{abstract}




\section{Acknowledgments}

I would like to express my deep gratitude to my advisor Professor Erik Bodegom, for the guidance that helped me immensely in the preparation of this dissertation, and for allowing me to grow as a research scientist.

I am heavily indebted to Dr. Ricardo Ascazubi, who acted as a mentor and supporter for my work in soft errors. Dr. Ascazubi was instrumental in helping me complete this work, and I could not have imagined having a better mentor for my Ph.D. study. He provided me an opportunity to join his team as an intern, and gave me access to the laboratory. His guidance helped me in all the time of research and writing of this thesis.

I would like to thank my committee members, Dr. Ralf Widenhorn, Dr. Raj Solanki and Dr. Branimir Pejcinovic for their insightful comments that helped me polish this thesis. I would also like to express special thanks to the members of Dr. Ascazubi's team at Intel corp., Dr. Scott Johnson, Dr. Curtis Shirota, Dr. Georgia Modoran and Dr. Norbert Seifert who patiently helped me by providing their experience and knowledge in the field. I would like express my gratitude to my family, who have encouraged me to continue my studies. I wouldn't be where I am today if it was not for the constant support of my mother, Gity. I would like to greatly express appreciation to my beloved partner Mark for his patience, encouragement and inspiration throughout this work, for all the sacrifices he made while I was working on this research, and for all the extra help he gave me with our son Henry.

Finally, I would like to acknowledge the support of Semiconductor Research Corporation (SRC) for their financial support and Intel Corporation for granting me lab access. 


\section{Table of Contents}

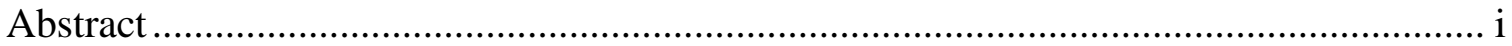

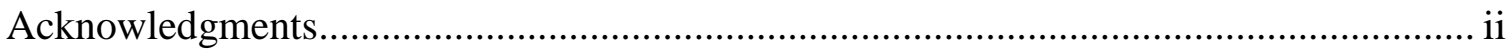

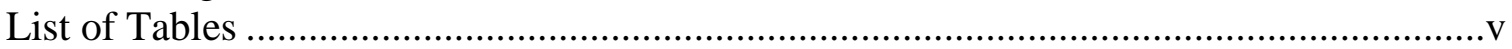

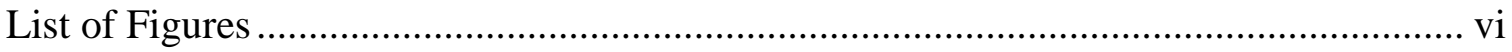

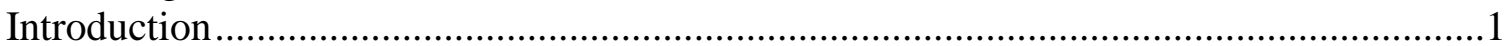

Chapter 1 - Overview and Background Information .................................................4

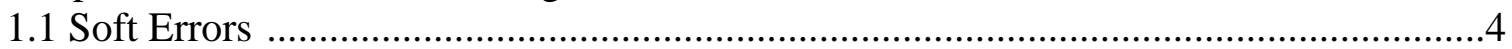

1.2 Effects of Soft errors on Computer Components..................................................6

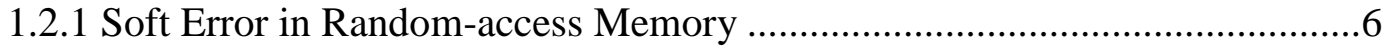

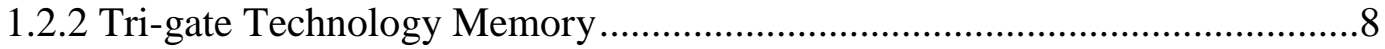

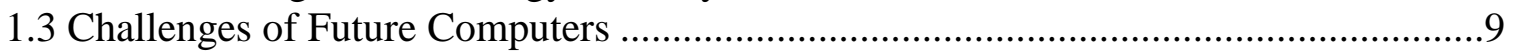

1.4 Ionizing radiation in Silicon Computers ................................................................ 10

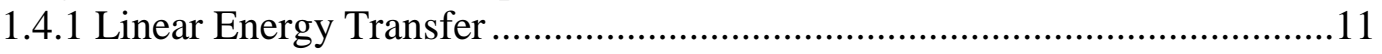

1.4.2 The Funneling Effect .................................................................... 11

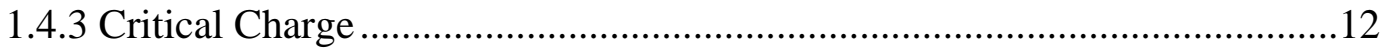

Chapter 2 - Absorption Processes in Semiconductors ................................................14

2.1 Fundamental Indirect Absorption (Interband Absorption) ....................................15

2.2 Free Carrier Absorption (Intraband Absorption) ................................................16

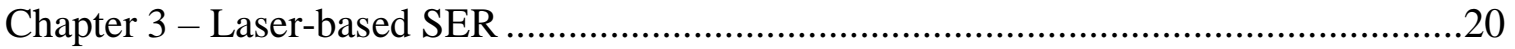

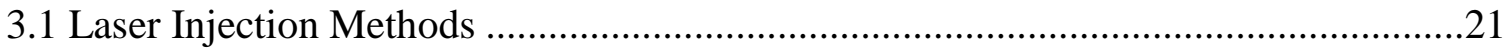

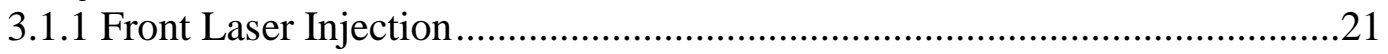

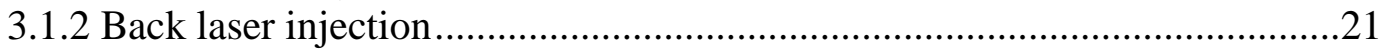

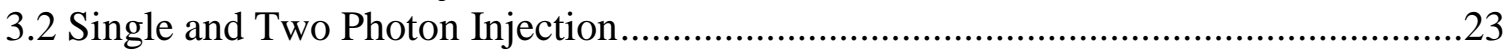

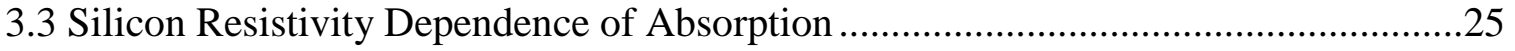

3.4 Measurements of Absorption Coefficient of High and Low Resistivity Si ................25

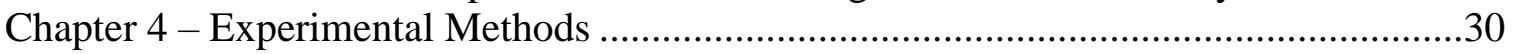

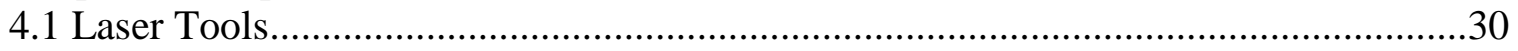

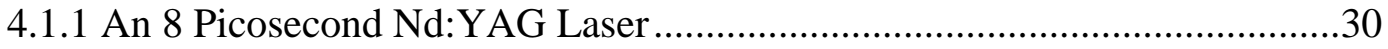

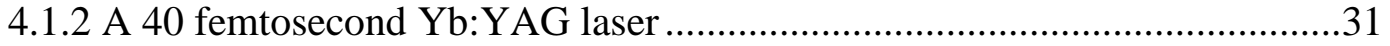

4.1.3 A compact fiber based femtosecond laser ...............................................33

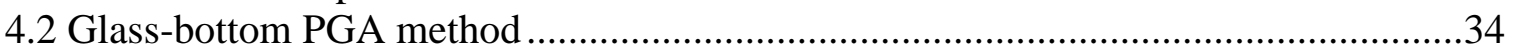

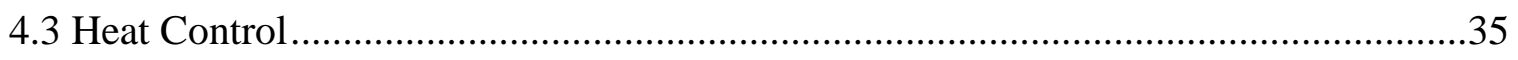

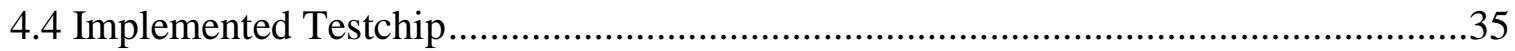

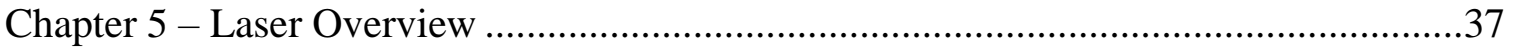

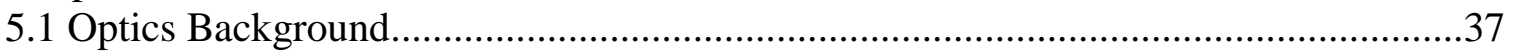

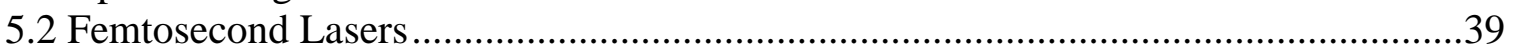

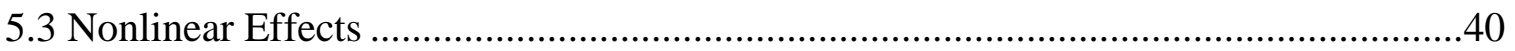

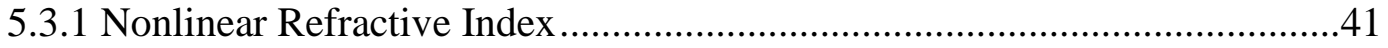

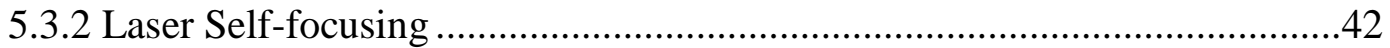

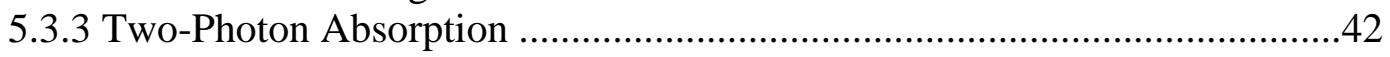

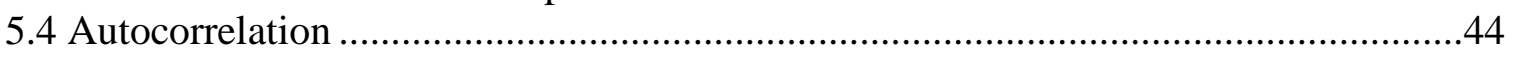

Chapter 6 - Laser-Particle Beam Correlation ..............................................................48 


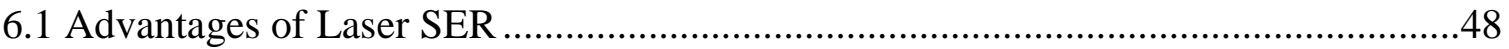

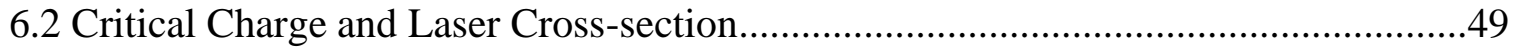

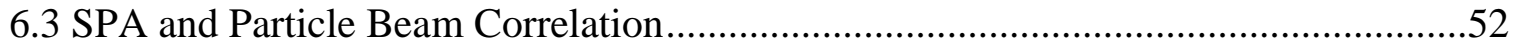

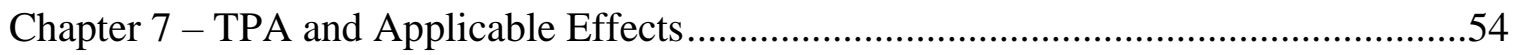

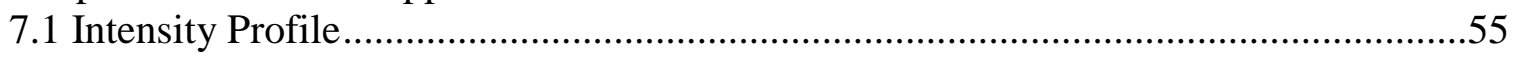

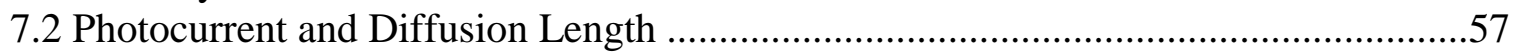

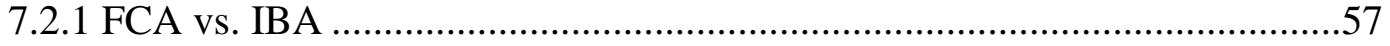

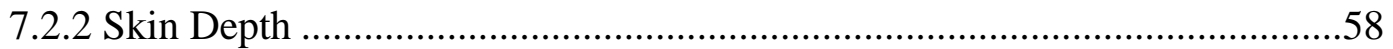

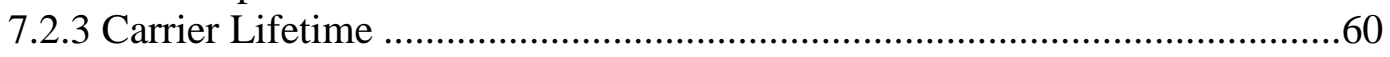

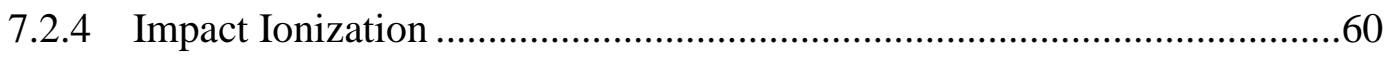

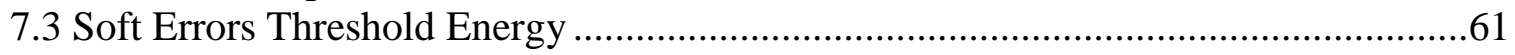

7.3.1 Threshold Energy at Different Thicknesses............................................62

7.3.2 Laser and Beam Correlation ...................................................................6

7.3.3 Total Number of Flips of $\mathrm{N}$ and P-type Devices ......................................68

Chapter 8 - Experiments and Results of Laser Testing ............................................. 70

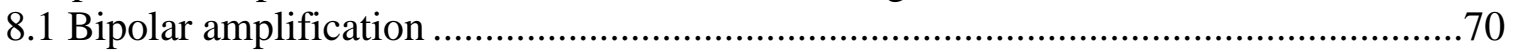

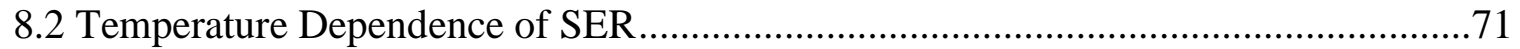

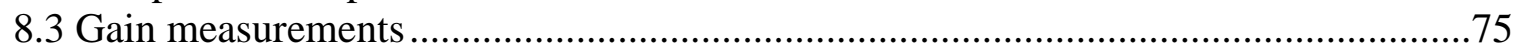

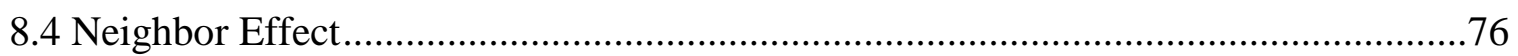

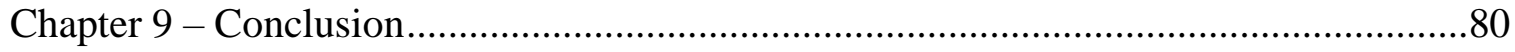

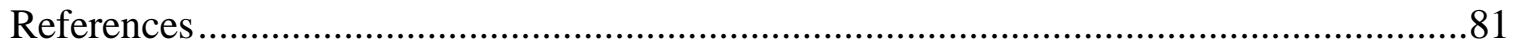




\section{List of Tables}

Table 1 - Power reading of 1040nm and 1550nm lasers ............................................25

Table 2 - Absorption percentages for IBA and FCA of HR and LR samples .................27

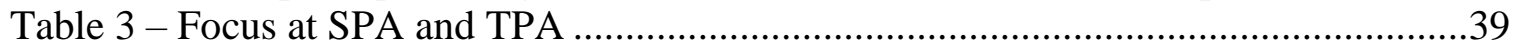

Table 4 - Comparison of charge created by particle beam and laser ..............................51

Table 5 - Comparison of experimental and theoretical absorption coefficients ...............57 


\section{List of Figures}

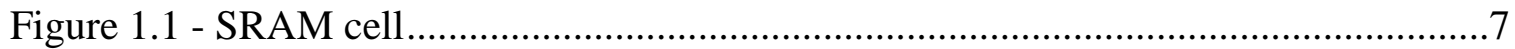

Figure 1.2 - Planar (left) and tri-gate transistor structure .........................................

Figure 1.3 - Planar (left) and tri-gate (right) design. (Intel's 22nm technology 2011) .......9

Figure 1.4 - SRAM Planar and tri-gate SER comparison (Seifert IEEE 2016) ................10

Figure 1.5 - Charge generation and collection phases in a reverse-biased junction (Baumann, IEEE 2005)

Figure 2.1 - Figure 2.1 - Photon induced transitions, Deen M. Jamal Kumar Basu,

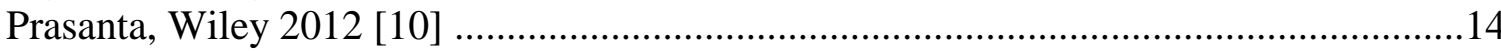

Figure 3.1 - Front laser injection vs. back laser injection ..........................................20

Figure 3.2 - Metal-1 through metal-8 layer cross-section Ingerly IEEE 2012 [16] ........21 Figure 3.3 - Absorption coefficients of different semiconductors - Deen M. Jamal Kumar

Basu, Prasanta, Wiley 2012 [10] ............................................................................22

Figure 3.4 - Quantum efficiencies of semiconductors, PV Measurements, Inc. 2014 [20]

Figure 3.5 - Alpha (SPA, green) vs. Beta (TPA, red) absorption as a function of wavelength. This data was collected using a variable fs laser tool. ................................24

Figure 3.6 - Power measurement of the bulk sample..................................................26

Figure 3.7 - IBA and CFA Diagram based on laser wavelength ..................................28

Figure 3.8 - IBA and FCA of Silicon, D. K. Schroder, IEEE 1978 [19] ..........................29

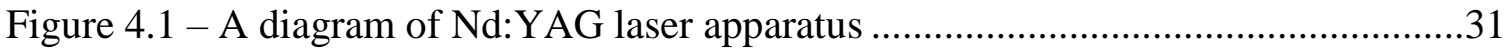

Figure 4.2 - $1040 \mathrm{~nm}$ (a) and $1550 \mathrm{~nm}$ (b) laser experiment setups. They both setups

sample can move along a plane perpendicular to laser beam. .......................................32

Figure 4.3 - Pump-probe setup. Red path indicates the laser beam used in SPA

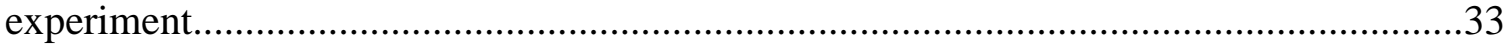

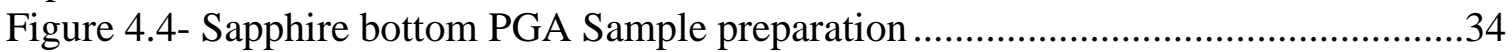

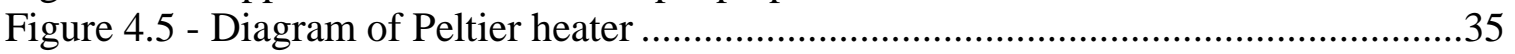

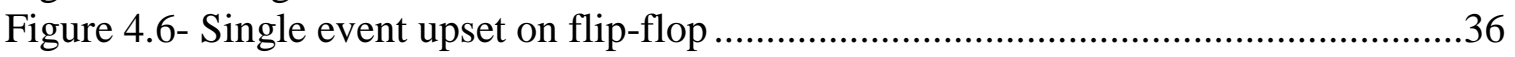

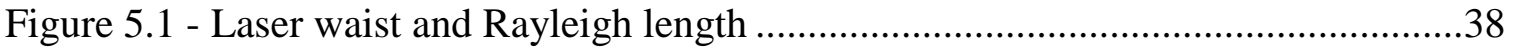

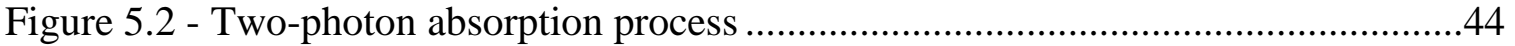

Figure 5.3 - Autocorrelation (left) and our modified version setup (right) .....................45

Figure 5.4 - Current signal in time for our simplified version of autocorrelation .............46

Figure 5.5 - Laser beam power at minimum (left) and at peak (right) ...........................47

Figure 6.1 - Laser and beam correlation, Ascazubi IEEE 2013 [17] ..............................48

Figure 6.2 - Neutron differential flux as a function of neutron energy, Gordon IEEE 2004

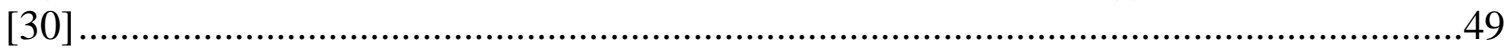

Figure 6.3 - Example of a typical energetic neutron of $100 \mathrm{MeV}$ that causes upset in a

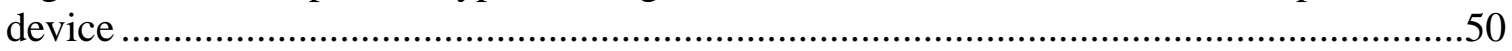

Figure 6.4 - Energy band structure of silicon - Sze p.14 2007 [35] ................................51

Figure 6.5 - Laser spot size compared to device area .................................................52

Figure 6.6 - Beam and $1040 \mathrm{~nm}$ laser correlation - NMOS (left) and PMOS (right) -

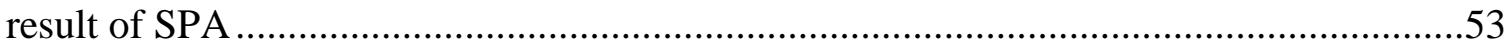

Figure 7.1 - SPA, TPA and beam energy transfer diagram......................................54 
Figure 7.2 - 1040nm and 1550nm lasers intensity profile

Figure 7.3 - Laser focus inside the sample at which TPA coefficient is at maximum in compare to SPA coefficient which is constant

Figure 7.4 - Photocurrent vs. thickness, 1040nm (left) vs 1550nm (right)......................57

Figure 7.5 - Diffusion length and carrier lifetime as a function of carrier density - Tyagi

$1983[32]$

Figure 7.6 - Laser scanning pattern with respect to testchip layout...............................61

Figure 7.7 - Threshold energy vs sample thickness for HR sample using 1040nm (red)

and $1550 \mathrm{~nm}$ (blue) lasers

Figure 7.8 - Total number of flips as a function of laser power for $30 \mu \mathrm{m}$ (left) and $80 \mu \mathrm{m}$ (right) thick samples. The top and bottom row results are from $1040 \mathrm{~nm}$ and $1550 \mathrm{~nm}$ lasers respectively .....

Figure 7.9 - Calculation of the volume of a frustum.

Figure 7.10 - Demonstration of charge generation following each laser pulse ................65

Figure 7.11 - A diagram of LR vs. HR as the laser beam penetrates...............................66

Figure 7.12 - Comparison between HR (green and blue) to LR (red and orange)

intensities

Figure 7.13 - SPA and TPA vs. beam cross-section

Figure 7.14 - Number of flips as a function of laser power for $1040 \mathrm{~nm}$ (left) and 1550

nm laser (right)

Figure 8.1 - Parasitic bipolar elements. The NMOS device has a lateral parasitic NPN bipolar transistor and the PMOS device has a lateral parasitic PNP bipolar transistor. (Oluwole A. Amusan, IEEE 2006 [21])

Figure 8.2 - Threshold energy of NMOS (left) and PMOS (right) latches at different

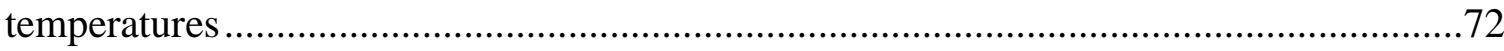

Figure 8.3 - NMOS and PMOS drain current as a function of Laser power ...................72 Figure 8.4 - Parasitic bipolar effect in a PNP structure - Laser testing ...........................75

Figure 8.5 - Gain at different temperatures of PNP - Electrical testing ..........................76

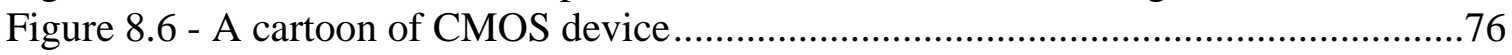

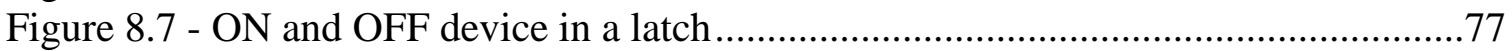

Figure 8.8 - PMOS devices in ON and OFF configurations with laser on ......................77 Figure 8.9 - Drain current for NMOS and PMOS devices on the ON configuration. 1

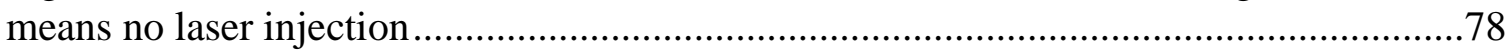

Figure 8.10 - Carrier path for NMOS (left) and PMOS (right) devices ..........................78 


\section{Introduction}

The negative effect of radiation on electronic devices has been a major concern of scientists and chip designers since it was first observed in the 60s. A high-profile example affected what was the second fastest supercomputer in the world in 2002, a machine called ASCI Q at Los Alamos National Laboratory. When it was first installed at the New Mexico lab, this computer could not run more than an hour or so without crashing. The problem was that an address bus on the microprocessors found in those servers was unprotected, meaning that there was no check to make sure the information carried on these within-chip signal lines did not become corrupted. And that is exactly what was happening when these chips were struck by cosmic radiation, the constant shower of particles that bombard Earth's atmosphere from outer space. Cosmic rays are a fact of life, and as transistors get smaller, the amount of energy it takes to spontaneously flip a bit gets smaller, too. By 2023, when exascale computers, ones capable of performing $10^{18}$ operations per second, are predicted to arrive in the United States, transistors will likely be a third the size they are today, making them that much more prone to cosmic ray-induced errors. For this and other reasons, future exascale computers will crash more frequently than today's supercomputers. The exact rate of errors affecting modern memory is a matter of some debate, and according to a 2009 research performed on a supercomputer called Jaguar in Tennessee, cosmic ray-induced errors happen at a rate of 350 per minute. [1] To further advance our computers, radiation-related reliability concerns affecting semiconductors need to be clearly understood. The successive steps involved in the SER process are:

- Interaction of radiation with matter 
- Ionization mechanism

- Collection of charges

- Understanding the effects on semiconductor structures and overall systems

Despite the extensive research that has been done to investigate the mechanisms involved, little is known regarding the radiation effects in modern technology. Our goal is to better understand the issues regarding the reliability of integrated circuits. This thesis reports the work done in demonstrating the use of an ultrashort pulsed laser as a tool to study the effects of cosmic rays in computers. The effects of the device temperature are investigated later, the results are presented, and an explanation is offered. Further, a new finding called the neighbor effect is presented which is expected to shine a new light on the subject. The thesis is divided into eight chapters. The description of their contents is presented below:

Chapter 1: An overview of soft errors with a short history and the method of calculating it is presented. The effects of soft errors in computers is then explained. Later, the mechanisms involved in ionizing radiation in silicon are explored.

Chapter 2: Different processes involved in absorption of photocurrent in silicon will be noted. Interband absorption will be reviewed first. Next, free carrier absorption will be discussed, and a classical model for free carrier absorption as a function of wavelength will be presented.

Chapter 3: Laser as an alternative and complementary tool to study soft error effects will be presented in this chapter. Preferred laser injection method for the work done in this work will be discussed. Single and two photon absorptions processes will be presented in relevance to laser injection method, and the advantage of two-photon 
process will be reviewed. We will discuss silicon bulk resistivity and its role in the absorption processes later in this chapter.

Chapter 4: Our three different laser tools used for this work will be presented in this chapter. I present here a method we developed to place the thinned samples in the path of laser beam using pin grid array packages. Additionally, temperature control method used for samples will be discussed.

Chapter 5: This chapter includes a review of some background regarding lasers. The nonlinear optical effects such an intensity-dependent refractive index, that are relevant to this work will be summarized. An overview of two-photon absorption (TPA) process and our method of verifying TPA will then be presented.

Chapter 6: This chapter will discuss the advantages of laser over particle accelerator testing. An analysis of beam method of defining the critical charge be viewed and compared to that of the laser-based method. Finally, single photon absorption (SPA) results will be compared to the beam data.

Chapter 7: This chapter will present and examine the TPA related data. TPA crosssection will be compared to beam cross-section at the end of the chapter.

Chapter 8: In addition to direct study of soft error rate (SER), laser provides an attractive tool to study other effects in semiconductors. In this chapter, we will present a few of these findings such as temperature effect and the neighbor effect. Chapter 9: This chapter will summarize our results, and will discuss further work planned. 


\section{Chapter 1: Overview and Background Information}

In this chapter, we will describe soft errors and the events responsible for their occurrence. We will then go over the effects of soft errors in computer components, and specifically static random-access memories. Challenges facing the future of computation will be reviewed next. Finally, the mechanisms involved in the soft error will be discussed.

\section{$1.1 \quad$ Soft Errors}

When transistors are exposed to ionizing radiation, electron-hole pairs are created throughout the semiconductor material. Some of the carriers generated near a junction will traverse the junction and produce transient current at the device terminals. This transient current can modify a stored value, causing a change in the stored information and lead to a soft failure of the device. A soft failure or soft error is defined as a spontaneous change in stored information. This kind of error is normally recoverable and does not destroy the device. These errors can manifest themselves as anything from a simple missing or incorrectly colored pixel on a computer monitor to a drastic server crash. Single-event upsets (SEUs) refer to errors caused by a hit from a single particle. Energetic particles from decay of radioactive atoms or extraterrestrial cosmic rays are the main source of such electronic disturbance.

SEUs are induced by the interaction of an ionizing particle with electronic components. Ionizing particles can be primary such as heavy ions in space environment or alpha particles produced by radioactive isotopes contained in the die or its packaging, or secondary created by the nuclear interaction of a particle, like a neutron or a proton with 
silicon, oxygen or any other atom of the die.[2] Alpha particles of terrestrial origin produced by the decay chain of uranium or thorium atoms are one source of device soft failure. Evidence of soft errors from impact of alpha particles were first discovered in 1978. May and Woods of Intel published their results associating the compromised data of certain products to trace radioactivity found in memory packaging materials. After the memory had been written, the values of some of the bits had randomly changed. At the time, some materials such as sealing glasses, fillers and alumina contained several parts-per-millions of radioactive isotopes uranium-238 and thorium-232. These small quantities were sufficient to produce a flux of alpha particles leading to soft errors. Soft error rate (SER) due to alpha particles can be reduced by improving the purity of materials and by various shielding methods. An alpha particle can cause a sudden generation of electrons on the order of millions in a semiconductor over a path length of a few microns. [3]

In the atmosphere, particles are generated in collisions between galactic cosmic rays and air molecules, which consist of atomic nuclei of nitrogen and oxygen. This creates a cascade of particles, including pions, muons, electrons, gamma rays, protons, and neutrons. Neutrons are considered to be the major contributor to SEUs in semiconductors at terrestrial altitudes, due to their relatively high flux, low attenuation, and long lifetime. [4] In 1979 Ziegler and Lanford published their results explaining the mechanism by which cosmic rays could cause upsets in electronics in a manner similar to alpha particles. Typically, what reaches sea level are high energy neutrons and protons as well as transient pions and muons. The two particles that have been shown to cause significant disturbance within electronic devices are neutrons and pions. Later in 1979 the first experimental work 
was performed confirming Ziegler et al. findings. Proton and neutron beams were used to bombard chips, and it was observed that both beams resulted in similar fail rates, considering the number of particles and energy per particle. The soft error rate (SER) is the rate at which soft errors appear in a device for a given environment, and can be given in FIT (failure in time). The cross-section can be used to calculate SER of a device tested at a particle beam.

If, with the total beam fluence of $N$ neutrons $/ \mathrm{cm}^{2}, n$ errors are obtained in a device, the cross-section is

$$
\sigma=\frac{n}{N}
$$

With $\phi$ being the particle flux the FIT value is:

$$
F I T=\frac{n}{N} \times \phi
$$

SER is then determined by integrating the product of the bit fail cross section and the differential flux over the energy range where the circuit is susceptible to fails

$$
S E R=\int \sigma(E) \cdot\left(\frac{d \phi}{d E}\right) d E
$$

where $d \phi / d E$ is the differential flux. [2]

\subsection{Effects of Soft Errors on Computer Components}

\subsubsection{Soft Error in Random-access Memory}


Soft errors can manifest in a great variety of ways depending on the device design. In analog devices, Single Event Transients (SETs) also called Analog Single Event Transients (ASETs) are mainly transient pulses in operational amplifiers, comparators or reference voltage circuits, and since they can be recognized and filtered their effects are not severe.

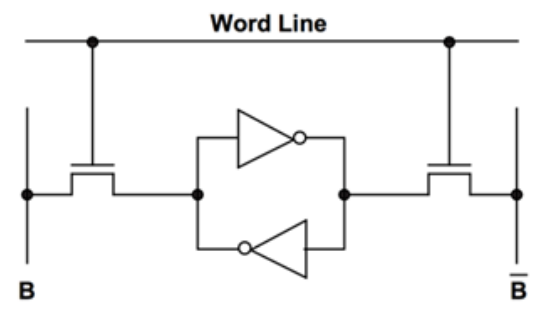

Figure 1.1 - SRAM cell

In high-speed input/output components soft errors can manifest as bit errors (bit error rate, BER), and are usually corrected by an error correction protocol. In combinatorial logic in which the output is only a function of the input present, SETs are transient pulses generated in a gate that may propagate in a combinatorial circuit path and eventually be latched in a storage cell such as a latch or a flip-flop. In memory devices, latches, and registers, soft errors are mainly called SEUs, and they correspond to a flip of the cell state. [2] SEUs in memories typically refer to the loss of information from a memory cell caused by a single ionizing particle. In a flip-flop or latch the information corruption is associated to the bit flip: $0 \rightarrow 1$ or $1 \rightarrow 0$ and is reversible, meaning the correct bit can be rewritten. A variety of error correction methods such as parity and error-correcting codes are available to detect and correct such errors. These methods cover all easy-to-implement places that an error could appear such as large cache areas. However, because they are expensive both in terms of chip real estate and speed, they are not utilized in small registers. Storage nodes like 
latches, flip-flops and random-access memory (SRAM) arrays are the most affected by SER, because unlike logic cells the error will be permanently recorded. [5]

Static random-access memories (SRAMs) are designed to provide a direct interface with the CPU for high speed data retrieval at low power consumptions. A typical SRAM cell is shown in Figure 1.1. The SRAM cell is connected to the internal circuitry by two access transistors. When the cell is not addressed, the two access transistors are closed and the data is kept in a stable state. These transistors are a combination of NMOS and PMOS that can store bits of data. [6] Reversed biased junctions of off-state MOSFET drains can collect charge produced by a single ionizing particle that can lead to a SRAM cell bit flip.

\subsubsection{Tri-gate Technology}

The development of tri-gate technology has led to a significant improvement in performance and energy efficiency of transistors. Unlike traditional 2-D planar transistors that form a conducting channel in the silicon region under the gate electrode when in the "on" state, in 3-D tri-gate transistors the current is controlled by using a gate on each of the three sides of the fin, two on each side and one across the top, providing a more effective field by eliminating the edge effect (Figure 1.2).
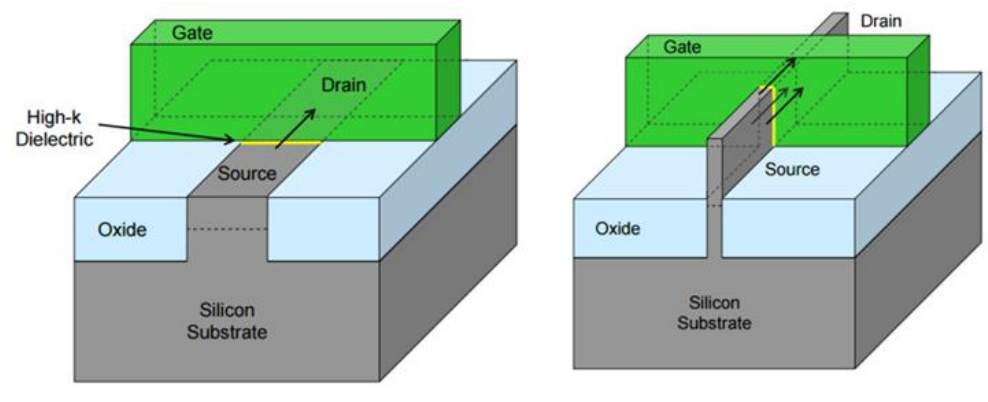

Figure 1.2 - Planar (left) and tri-gate transistor structure 
The additional control enables as much transistor current flowing as possible when the transistor is in the 'on' state, which maximizes the device performance, and as close to zero as possible when it is in the 'off' state to minimize power usage. Additionally, it enables the transistor to switch very quickly between the two states which also improves performance. They can have multiple fins connected together to increase total drive strength for higher performance (Figure 1.3). [7] Test results by Seifert et. al. show a decrease in SER in tri-gate over planar technology mainly due to device geometry (Figure 1.4). [8]

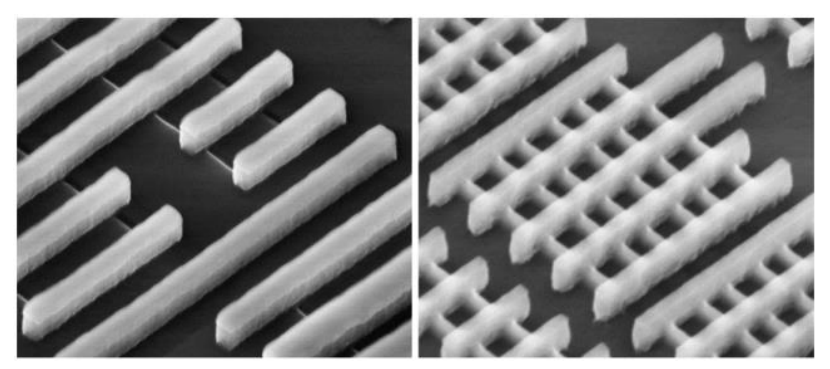

Figure 1.3 - Planar (left) and tri-gate (right) design (Intel's 22nm technology 2011)

\subsection{Challenges of Future Computers}

The remarkable improvements in computational power today is driven by sophisticated integrated circuits in microprocessors. These advancements have been resulted in fulfillment of Moore's law, which states transistor density doubles every two years, whereby ever more capable computing architectures have been enabled by smaller, faster, and cheaper fundamental microelectronic building blocks. [9] In regards to the effect of Moore's Law on radiation induced SERs, there are a few competing effects to consider. As 
transistor dimensions' scale down to submicron levels, the amount of energy it takes to spontaneously flip a bit gets smaller.

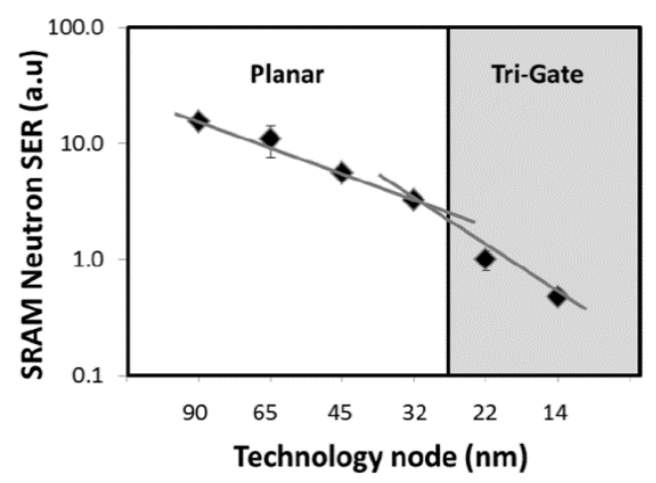

Figure 1.4 - SRAM Planar and tri-gate SER comparison (Seifert IEEE 2016)

At the same time, to create faster, more powerful computers the number of components within a system will need to increase by a large factor. There is also the mandate to keep the computers as energy efficient as possible, and this power savings will have to come from smaller transistors running at the lower voltages. Running at the edge of what it takes to make a transistor switch on or off increases the probability of circuits flipping states spontaneously. [1] Even though a decrease in the supply voltage makes electronic components more vulnerable to charge collection due to particle strikes, a smaller device size means reduction of the sensitive cross-section area on the chip, which will decrease the probability of particle collisions. Additionally, as components density rises, the probability of particle hit on the device increases. Currently it is not clear which is the dominating effect and further studies are required to better understand this.

\subsection{Ionizing radiation in Silicon}

\subsubsection{Linear Energy Transfer}


SEUs are caused by bursts of excess energy, creating electron-hole pairs, generated at locations where a collision between radiation and the silicon occurs in a semiconductor substrate. The charge is collected by the drain of the MOS transistors. Ionization is due to the Coulomb interaction between the incoming particles and silicon atoms. The magnitude of the disturbance an ion causes depends on the linear energy transfer (LET) of that ion. LET is the average energy loss by the ion per unit length of the material transversed. In a silicon substrate, one electron hole pair is produced for every $3.6 \mathrm{eV}$ of energy lost by the ion. LET is an important parameter to quantify the sensitivity of devices. [2] Charge collection generally occurs within a few microns of the junction, thus the collected charge $\left(Q_{\text {coll }}\right)$ for these events is in the order of femto Coulomb depending on the type of ion, its trajectory, and its LET. The generation of free careers follows the ion penetration, but it is usually considered instantaneous with the valid assumption that the response of the device is much slower than the carrier generation time.

\subsubsection{The Funneling Effect}

During the collision or interaction between ion and substrate, a cylindrical track of electron-hole pairs with a very high carrier concentration is formed along the path of the energetic ion (Figure 1.5a). When the resultant ionization track crosses the depletion region or comes close to it, carriers are rapidly collected by the electric field creating a large transient current. Because of the action of the ionization track, the original field in the depletion region is distorted and elongated deeper along the track. This expansion of the field acts like a funnel for the charges, which is called the funneling effect. This funnel 
increases the efficiency of the drift collection by extending the high field depletion region deeper into the substrate (Figure 1.5b).

The size of the funnel is a function of substrate doping, and the funnel distortion increases for decreased substrate doping. Once this collection phase is completed, diffusion begins to dominate the collection process (Figure 1.5c). Additional charge is collected as electrons diffuse into the depletion region until all excess carriers have been collected, recombined, or diffused away from the junction area.

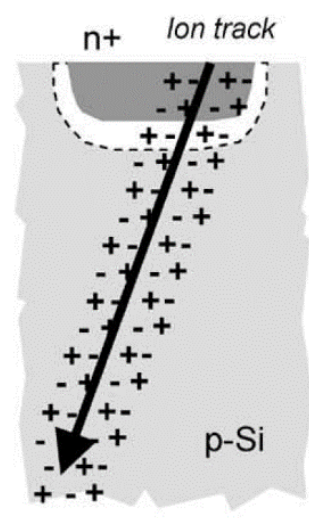

(a)

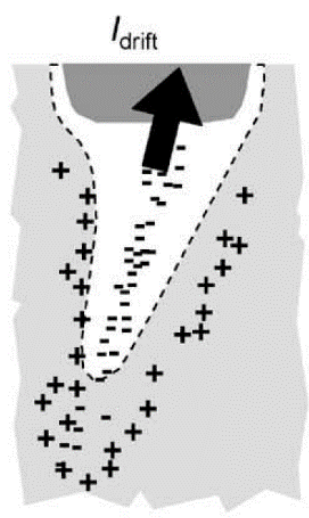

(b)

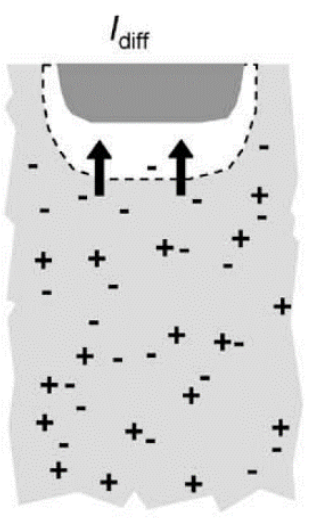

(c)

Figure 1.5 - Charge generation and collection phases in a reversebiased junction (Baumann, IEEE 2005)

\subsubsection{Critical Charge}

A node is never isolated but is actually part of a collection of nodes in close proximity to each other. Therefore, charge sharing among nodes and parasitic bipolar action (the formation of an unintentional bipolar transistors between junctions and wells, refer to section 8.1) can greatly influence the amount of charge collected. The sensitivity of a device must be described by a parameter that allows a simple evaluation whether a collision can disturb the electronic function. The simplest parameter to consider is the charge 
collected at a given node and to compare its value to the minimum charge needed to upset the function. This minimum charge is called the critical charge $Q_{c r i t} . Q_{c r i t}$ is the charge carrying an elementary information in the component. SEUs occur when the total charge of carriers liberated by an ionizing particle and instantaneously collected at a junction is greater than the $Q_{\text {crit }}$. [2]

Soft error and the challenges facing future of computation where covered in this chapter. Absorption processes in silicon related to our work will be reviewed in the next chapter. 


\section{Chapter 2: Absorption Processes in Semiconductors}

In this chapter, we will go over different processes involved in absorption of photocurrent in silicon. Interband absorption will be reviewed first. Next, free carrier absorption will be discussed, and a classical model for free carrier absorption as a function of wavelength will be presented. Carrier concentration is a major factor in Si absorption; hence the effects of different carrier concentrations will be viewed. Additionally, the results of an absorption experiment will be presented at the end of this chapter.

The intensity of the electromagnetic radiation $I(z)$, incident on the surface of a semiconductor is reduced along the depth due to absorption in the material according to the Beer's Law

$$
I(z)=I_{0} \exp (-\alpha z)
$$

where $\alpha$ is called the absorption coefficient of the material, $I_{0}$ is the intensity of the incoming wave and $z$ is the depth of the material.

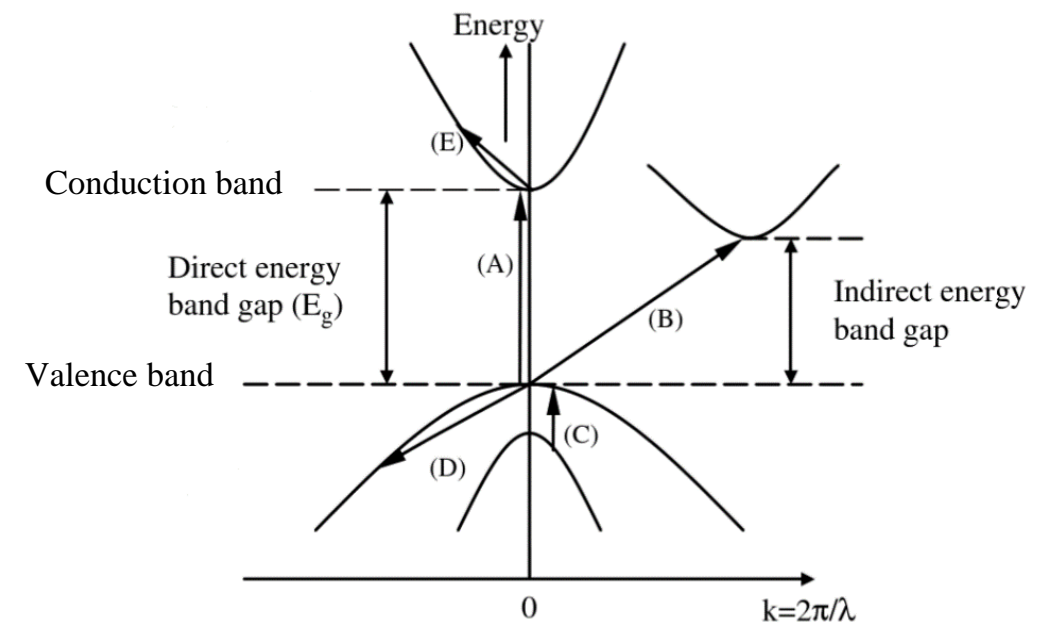

Figure 2.1 - Photon induced transitions, Deen M. Jamal Kumar Basu, Prasanta, Wiley 2012 [10] 
When radiation is incident on semiconductors, photons induce transitions among different energy levels in different bands in the material as shown in figure 2.1. [10] The energy of photons can be transferred to the lattice phonons, sending carriers from valence band (VB) to the conduction band (CB), generating an electron-hole pair during the process. Photons with energy below the material's bandgap cannot be absorbed, and the semiconductor is transparent to these photons. Such energies typically correspond to light frequencies below the visible and infrared spectrum for semiconductors. The process (A) in Figure 2.1 represents direct valence to conduction band transitions that occur in a direct band gap semiconductor, like GaAs. Process (B) illustrates indirect valence to conduction band transitions aided by photon-phonon coupling interactions as in silicon. Process (C) represents intervalence band transitions. Process (D) depicts valence band free-carrier transitions aided by impurities or photon-phonon interactions. Process (E) is for free-carrier transitions in a conduction band aided by impurities or photon-phonon interactions. Depending on the photon energy, a single type or a combination of a few types of all the absorption processes indicated in the figure 2.1 may be important. [10] The specific mechanisms by which the absorption occurs will depend on the type of material. Silicon is the substrate material in our testchip, hence the absorption processes related to $\mathrm{Si}$ will be discussed here.

\subsection{Fundamental Indirect Absorption (Interband Absorption)}

Interband transitions that occur between the $\mathrm{VB}$ and $\mathrm{CB}$ can either be vertical, undergoing only a change in energy, or diagonal, undergoing a change in both energy and momentum. 
In either case, both energy and momentum must be conserved for the overall process. In $\mathrm{Si}$, a photon cannot directly lift an electron to the conduction band. The absorption process in Si then involves an electron, a photon, and a phonon. In order to conserve momentum, quantized lattice vibrations act as a momentum-conserving agent. The photon brings an electron in the valence band to an intermediate state. The electron then gains momentum by colliding with a phonon and comes to one of the six degenerate conduction band valleys. Due to point symmetry of the face-centered cubic (fcc) lattice of Si the six conduction band valleys are equivalent. The probability of this transition which is a lot smaller than direct bandgap transition, can be calculated from second order perturbation theory. [26]

\subsection{Free Carrier Absorption (Intraband Absorption)}

Free carrier absorption involves the transfer of photon energy to an electron or hole in the semiconductor conduction or valence band, respectively. Excited electrons transition to a state in the same or another conduction band. Holes make similar transitions in the valence bands. Naturally, free carrier absorption is stronger in semiconductors with high electron or hole concentrations, such as heavily doped or highly injected silicon. At near-bandgap photon energies, where band-to-band absorption is weak, free carrier absorption can contribute significantly to the total absorption in the semiconductor. In the case of silicon, the onset of this effect is in the near infrared (photon wavelengths above $\sim 1000 \mathrm{~nm}$ ). Free carrier absorption is usually identified as a parasitic process it has a negative impact on the performance of devices such as silicon solar cells, thermophotovoltaic cells, and infrared photodetectors. [11] 
Free-carrier absorption (FCA) is the result of direct absorption of photons by the carriers in the conduction or valence band. These transitions are shown in figure 2.1 as process (D) and process (E), respectively, in the valence band (free holes) and conduction band (free electrons). [26] FCA does not produce ionization, but increases the energy of carriers within these bands. This also requires a change in momentum, which is provided by interaction with the lattice through phonon interaction or by scattering from ionized impurities.

The expressions for the free-carrier absorption coefficient may also be obtained by using classical electromagnetic theory and the Drude model for conduction. The motion of a free electron in the conduction band of a semiconductor under the influence of a sinusoidal electric field can be written as

$$
m^{*} \frac{d^{2} x}{d t^{2}}+m^{*} g \frac{d x}{d t}=-e E_{0} \exp (j \omega t)
$$

where $\mathrm{x}$ is the displacement of the carrier having effective mass $m^{*}, g$ is the damping coefficient, and $E_{0}$ is the amplitude of the electric field varying with angular frequency $\omega$. The first term is the force term, the second term represents the damping of electron motion by scattering with phonons and impurities, and the right-hand side represents the applied force. The steady-state solution of the above equation is

$$
x=\frac{\left(e E_{0}\right) / m^{*}}{\omega^{2}-j \omega g} \exp (j \omega t) .
$$

If $N$ denotes carrier concentration per unit volume, then the displacement of carriers $x$ will produce additional polarization $P_{1}$ given by 


$$
P_{1}=-N e x
$$

The total polarization is

$$
P=P_{0}+P_{1}
$$

where $P_{0}$ is the polarization without free carriers present. The relative permittivity is given by

$$
\varepsilon_{r}=\frac{\varepsilon}{\varepsilon_{0}}=1+\frac{P}{\varepsilon_{0} E}=n_{0}{ }^{2}+\frac{P_{1}}{\varepsilon_{0} E}
$$

where $n_{0}$ is the refractive index without free carriers. Using expressions for $P_{1}$ and $x$ from above we can write relative permittivity as

$$
\varepsilon_{r}=n_{0}{ }^{2}-\frac{\left.\left(N e^{2}\right) / m^{*} \varepsilon_{0}\right)}{\omega^{2}-j \omega g}
$$

The real and imaginary parts of relative permittivity are

$$
\begin{gathered}
\varepsilon_{r r}=n_{0}{ }^{2}-\frac{\left.\left(N e^{2}\right) / m^{*} \varepsilon_{0}\right)}{\omega^{2}+g^{2}} \\
\varepsilon_{r i}=\frac{\left.\left(N e^{2} g\right) / m^{*} \omega \varepsilon_{0}\right)}{\omega^{2}+g^{2}}
\end{gathered}
$$

Normally the value of $g$ is a few orders of magnitude smaller than $\omega$. At steady state

$$
\frac{d^{2} x}{d x^{2}}=0
$$

And the drift velocity is related to the mobility by the relation 


$$
\frac{d x}{d t}=\mu E
$$

This allows the expressions for real and imaginary relative permittivity to be simplified as

$$
\begin{gathered}
\varepsilon_{r r}=n_{0}{ }^{2}-\frac{N e^{2}}{m^{*} \varepsilon_{0} \omega^{2}} \\
\varepsilon_{r i}=\frac{N e^{3}}{\omega^{2} m^{* 2} \varepsilon_{0} \mu}
\end{gathered}
$$

The free carrier absorption coefficient $\alpha$ can be written as

$$
\alpha=\frac{k \varepsilon_{r i}}{n}=\frac{N e^{3} \lambda^{2}}{4 \pi^{2} n m^{* 2} \mu \varepsilon_{0} c^{3}}
$$

where $k$ is the wave vector, and $\mathrm{n}$ is the refractive index. As it can be seen free carrier absorption increases with carrier density with a wavelength dependence of $\lambda^{2}$.Additionally, equation 11 indicates that there is a change in refractive index due to free carriers that needs to be considered.

In laser experiments, FCA is a loss mechanism that increases absorption by Si bulk in highly doped regions. It can significantly reduce the light intensity in layers below highly doped regions, and must be taken into account.

This evaluation of absorption processes in $\mathrm{Si}$, especially the free carrier absorption discussed here, will help us make a theoretical estimation of free carrier absorption to compare to our experiment results later in chapter 6. 


\section{Chapter 3: Laser-based Soft Error Rate (SER)}

Using a tool that is both an alternative and complementary to a particle beam to study SER effects will be presented in this chapter. The preferred laser injection method for the work done in this dissertation will be discussed. Single and two photon absorptions processes will be discussed in relevance to laser injection method, and the advantage of two-photon process will be reviewed. We will inspect silicon bulk resistivity and its role in the absorption processes later in this chapter.

To better understand the SER mechanism and to confirm the reliability of the various hardening methods, ion beams in accelerators have been used to expose circuits to high energy particles. Particle accelerator testing has been the standard method to characterize the sensitivity of modern device technology to radiation. However, because the accelerator testing is expensive and not easily accessible, other techniques have been explored. A relatively recent approach for injecting faults from an external source is the use of laser beams. In was shown in 1963 for the first time that short pulses of radiation can be used to study the effects of ionizing particles in electronic devices. [13]

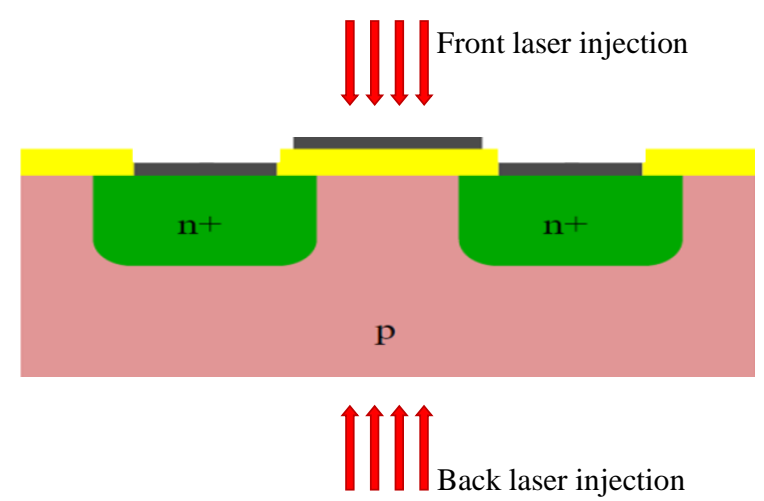

Figure 3.1 - Front laser injection vs. back laser injection 
Although there is a slight difference between the particle-material interaction and photonmaterial interaction, laser beam generated SERs have been shown to correlate well with particle beam testing. [17] Lasers can provide the ability to accurately deliver large amounts of energy into confined regions of a material. High levels of ionization can be achieved in semiconductor devices by irradiating the devices with short pulses of light. [14] Ultrashort laser pulses at desired energy allow for targeting specific area of a sample chip and injecting energy to the sample. The test chip can be targeted from front or back depending on the sample complexity and thickness (see figure 3.1).

\subsection{Laser Injection Methods}

\subsubsection{Front Laser Injection}

Front laser injection is a suitable method for simple testchips with thin metal layers. [15] $800 \mathrm{~nm}$ laser pulse is commonly used to probe simple transistors. However, this method is not beneficial for complex samples with interconnects, because the laser beam will be scattered and reflected of the metal layers and will not reach the junction (Figure 3.2).

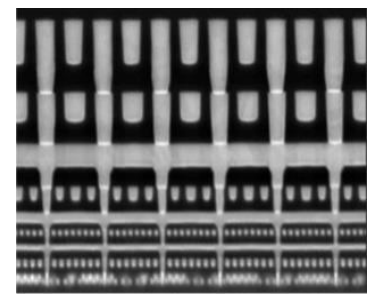

Figure 3.2 - Metal-1 through metal-8 layer cross-section Ingerly IEEE 2012 [16]

\subsubsection{Back laser injection}

For testing complex devices with advanced multilayer interconnect technology backside injection is the more appropriate method. The difficulty is that the laser beam must 
penetrate through the remaining bulk material to reach the device. To perform SER experiments samples need to be thinned.

The thicker the bulk layer the more laser energy will be absorbed. Beer's Law (equation 4) describes light absorption as a function of depth, and absorption coefficient. In order to penetrate to the device active area, bulk silicon needs to be thinned to a few tens of microns. For thinned samples, interband absorption is the only assumed carrier transport process for a typical high resistivity silicon sample (discussed in 3.3), and free carrier absorption is often neglected. In can become significant at longer wavelengths and high carrier concentrations. $1040 \mathrm{~nm}$ and $1064 \mathrm{~nm}$ wavelength lasers are suitable tools for backside injection. Figure 3.3 is a plot of interband absorption coefficient as a function of wavelength.

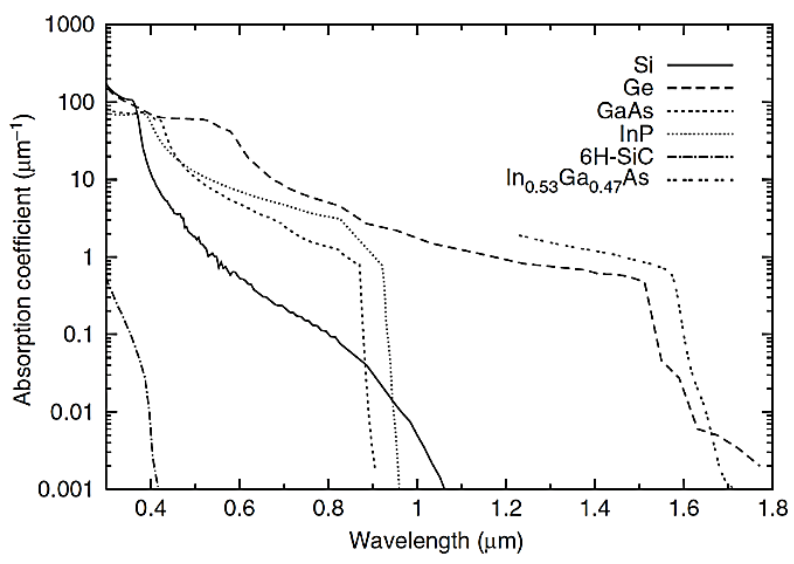

Figure 3.3 - Absorption coefficients of different semiconductors - Deen M. Jamal Kumar Basu, Prasanta, Wiley 2012 [10]

Another important factor to consider, once the laser beam reaches the junction, is the quantum efficiency $(\mathrm{QE})$. QE is a measure of how efficiently a material converts the incident light into electrical energy at a given wavelength. It is the ratio between the number of collected carriers and the number of all the incident photons on the device active area at 
a given wavelength. Figure 3.4 shows QE as a function of wavelength for different materials including Si. For example, QE of Si for 600nm laser beam is 95\%, meaning 95 out of every 100 photons will get absorbed. It can be seen that QE for Si quickly decreases at around 900nm wavelength.

Absorption coefficients, wavelength and carrier density are necessary to determine the amount of thinning required for a particular measurement. The thinning procedure itself introduces new challenges, as it adds an extra step to sample preparation process, and it is rather difficult to get an evenly thinned sample.

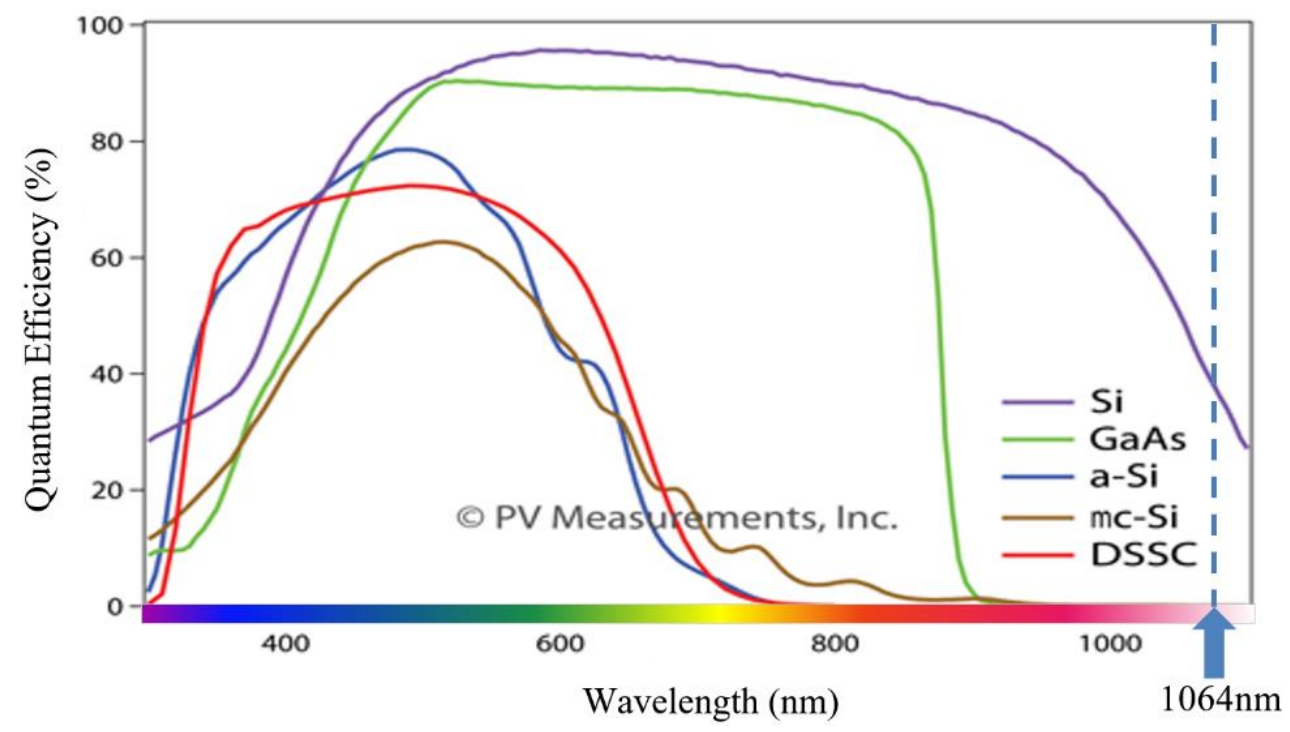

Figure 3.4 - Quantum efficiencies of a few semiconductors, PV Measurements, Inc. 2014 [20]

\subsection{Single and Two Photon Injection}

So far only single-photon absorption (SPA) has been considered in which each absorbed photon produces a single electron-hole pair. SPA decreases with wavelength of laser (Figure 3.5 green). Two-photon absorption (TPA) process is a non-linear effect that is a 
few orders of magnitude weaker than SPA, and therefore it is usually neglected. At high laser intensities, it becomes significant and must be considered. TPA is advantageous for our testing, because it eliminates bulk silicon interband absorption, and the bulk become practically transparent to the beam. This is because at higher wavelengths where TPA turns into a significant effect, laser energy is lower than the bandgap of $\mathrm{Si}$ and does not get absorbed. In this case thinning the sample will not be necessary.

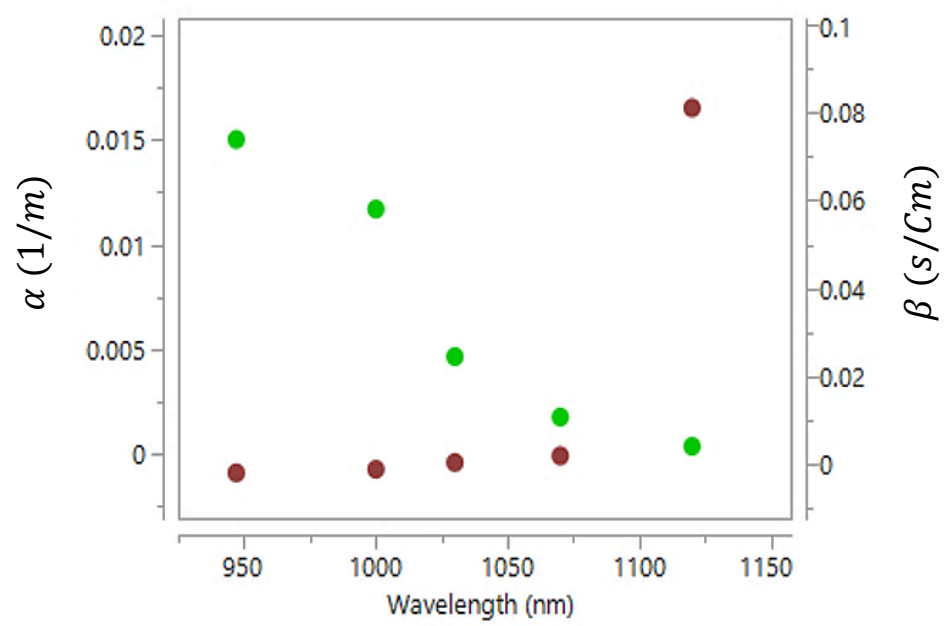

Figure 3.5 - Alpha (SPA, green) vs. Beta (TPA, red) absorption as a function of wavelength. This data was collected using a variable fs laser tool.

Free carrier absorption however, cannot be neglected due to higher wavelengths and must be considered. A laser in the near infrared range will the best choice in this regime, because even though the QE is nearly zero for infrared wavelength, transparency of the bulk sample to the beam will allow some of the laser energy to get deposited at the device. For a $1550 \mathrm{~nm}$ laser beam SPA will be near zero and TPA will dominate. Therefore, using a 1550nm laser will make this a more efficient process. Figure 3.5 is the data collected for linear absorption coefficient of low resistivity Si using our variable laser tool and predictions made for nonlinear absorption coefficient based on the first and second order effects. It will be shown 
later in this chapter that $1550 \mathrm{~nm}$ laser indeed is capable of carrying photocurrent through the silicon bulk at full thickness and target the metal layer below.

\subsection{Silicon Resistivity Dependence of Absorption}

Low resistivity silicon substrates are heavily doped giving them sheet resistivity of a few milliohm-cm and a carrier concentration of $\sim 10^{19}$ per cubic $\mathrm{cm}$. Heavy doping of substrates makes them highly absorptive in the near infrared due to band gap shifts, and this significantly affects the absorption processes taking place within the device. [18] The integration of RF circuits into CMOS devices favors a shift to a much higher silicon substrate resistivity achieved by low-doping of Si. High resistivity silicon substrate has resistivity of about $10 \mathrm{ohm}-\mathrm{cm}$, and carrier concentration of $\sim 10^{15}$. RF circuitry requires linear, analog devices with low noise and precision passive components. Very high substrate resistivity decreases capacitively-coupled cross-talk between digital, analog and $\mathrm{RF}$ components, improving noise isolation.

\subsection{Measurements of Absorption Coefficient of High and Low Resistivity $\mathrm{Si}$}

To verify the absorption coefficient equation discussed in section 2.2, high resistivity (high res or HR) and low-resistivity (low res or LR) samples of bulk only silicon (with no metal

\begin{tabular}{|c|c|c|}
\hline Sample Type & 1040nm Power Reading $(\mathrm{mW})$ & 1550nm Power Reading $(\mathrm{mW})$ \\
\hline HR1 $(30 \mu)$ & 4.57 & 4.85 \\
\hline HR2 $(31 \mu)$ & 4.65 & 4.92 \\
\hline LR1 $(38 \mu)$ & 4.95 & 3.42 \\
\hline LR2 $(38 \mu)$ & 3.78 & 3.64 \\
\hline
\end{tabular}

Table 1 - Power reading of 1040nm and 1550nm lasers 
layer) were prepared as will be described in section 4.2 and tested using the $1040 \mathrm{~nm}$ and 1550nm lasers (apparatus shown in figure 3.6).

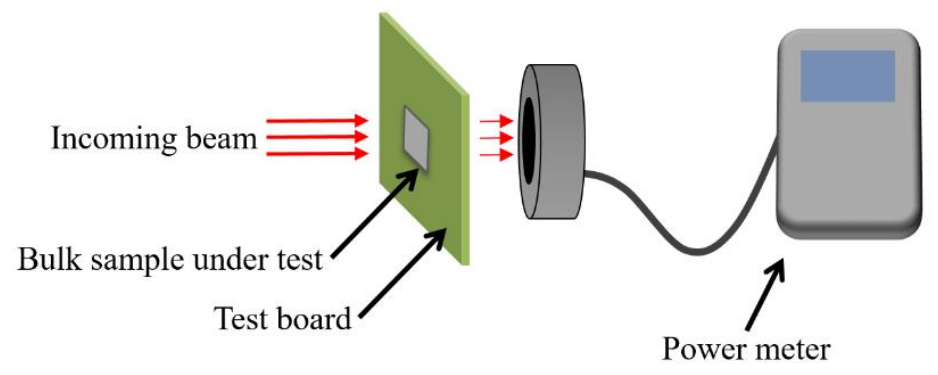

Figure 3.6 - Power measurement of the bulk sample

HR and LR samples have carrier concentration in the order of $10^{15}$ and $10^{19}$ respectively. The incoming beam of $1040 \mathrm{~nm}$ laser and $1550 \mathrm{~nm}$ laser were measured to be $7.76 \mathrm{~mW}$ and $7.31 \mathrm{~mW}$ respectively. Bulk samples of around 30 microns thin were targeted with laser beam of known power, and a power meter placed right behind the sample was used to measure the laser power after passing the bulk silicon (figure 3.6).

Table 1 is our power measurements for samples of high and low-res silicon.

These results are calculated after taking into account the reflection of the incoming beam off of air-sapphire interface, sapphire-silicon interface and silicon-air interface, using the Fresnel law and the continuity equation:

$$
R=\left|\frac{n_{1}-n_{2}}{n_{1}+n_{2}}\right|^{2}
$$

where $n_{1}$ and $n_{2}$ are refractive indices of the two media in consideration (air-sapphire, sapphire-Si and $\mathrm{Si}$-air). $R$ is calculated for each interface. 
It has been shown that interband absorption in Si decreases with increasing wavelength, and at wavelengths above 1200nm interband absorption is zero [19]. This can be seen from in our results in table 2 .

\begin{tabular}{|c|c|c|c|c|}
\hline Wavelength $\lambda(\mathrm{nm})$ & Sample & \% Laser absorbed & IBA & FCA \\
\hline 1040 & HR & $12 \%$ & $\checkmark$ & $\times$ \\
\hline 1040 & LR & $25 \%$ & $\checkmark$ & $\checkmark$ \\
\hline 1550 & HR & $1 \%$ & $\times$ & $\times$ \\
\hline 1550 & LR & $31 \%$ & $\times$ & $\checkmark$ \\
\hline
\end{tabular}

Table 2 - Absorption percentages for IBA and FCA of HR and LR samples

Although in the interband absorption (IBA) regime, free carrier absorption can be neglected, it can become significant at longer wavelengths and high carrier concentrations. According to the Drude classical model (equation 17), free carrier absorption increases rapidly with the square of wavelength.

The 1040nm laser is in the interband absorption regime (figure 3.7), and when it is incident on the HR sample, about $12 \%$ of the photocurrent is absorbed by IBA mechanism. Because of low carrier concentration in HR samples, there is no significant free carrier absorption. LR samples on the other hand have a few orders of magnitude higher carrier concentration which results in some FCA as it can be seen from table 2 . The $1550 \mathrm{~nm}$ laser is well beyond the IBA regime, and neither HR nor the LR samples are affected by this process. Free carrier absorption level is much higher for this laser, and LR samples with high carrier concentration are strongly affected as indicated in table 2. It has been shown that free carrier absorption (FCA) is important only for heavily doped semiconductors. [19] Figure 3.8 is a plot of carrier absorption as a function of wavelength for different hole concentration. It can be seen that IBA goes to zero for wavelengths greater than $1100 \mathrm{~nm}$, 
and FCA becomes very small for concentrations below $10^{17}$; hence FCA does not affect HR devices.

For 1550nm laser free carrier absorption coefficient for HR and LR samples can be calculated using equation $17\left(N_{H R}=10^{15}, N_{L R}=10^{19}\right)$ :

$$
\begin{gathered}
\alpha_{H R}=3.87 \times 10^{-2} \mathrm{~m}^{-1} \\
\alpha_{L R}=1.16 \times 10^{3} \mathrm{~m}^{-1}
\end{gathered}
$$

A free career absorption calculator was used to model the results here [21]. This calculator determines the fraction of infrared light absorbed by free carriers in a single pass through a semiconductor. The calculator determines the free-carrier absorption coefficient as a function of depth. This depends on the choice of semiconductor, the dopant concentration

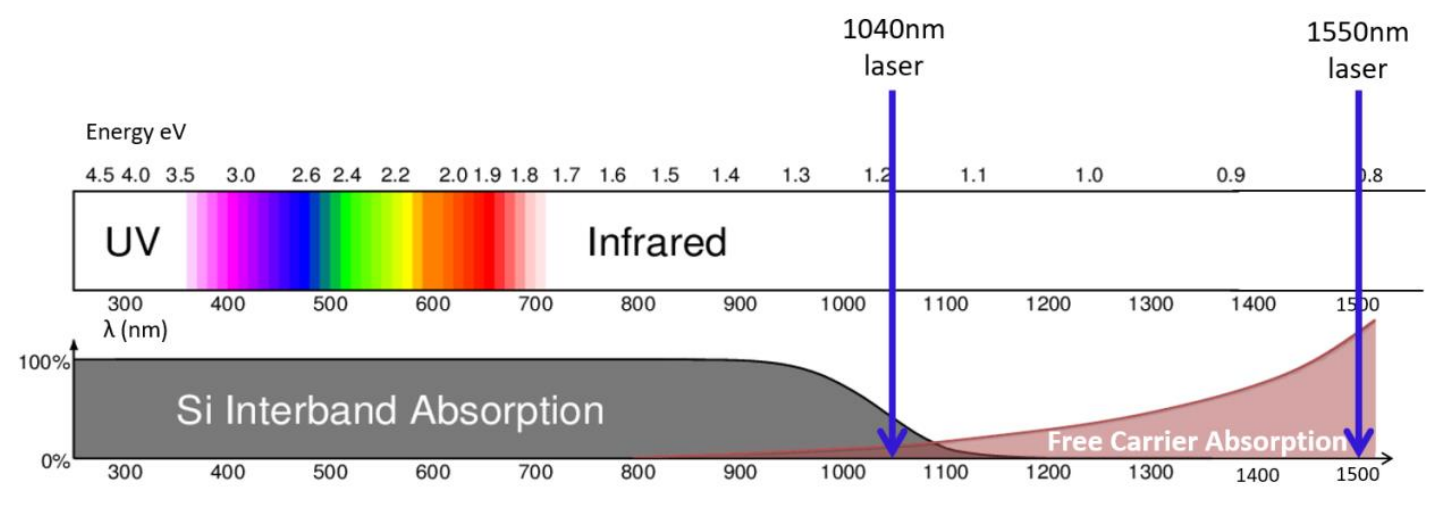

Figure 3.7 - IBA and CFA Diagram based on laser wavelength

and the wavelength of the incident light. The calculations assume that first, band-to-band absorption is negligible, and second, the free-carrier concentration equals the majoritycarrier concentration in equilibrium. The model for the free carrier absorption coefficient is derived from Baker-Finch [11] derivation which measures and models reflectance and transmittance dispersion to arrive at a parameterisation for the free carrier absorption coefficient that applies in the wavelength range between 1000 and $1500 \mathrm{~nm}$, and the range 
of dopant densities between $\sim 10^{18}$ and $3 \times 10^{20} \mathrm{~cm}^{-1}$. For LR type and 1040nm laser $\alpha_{L R}$ is about $16 \%$ compared to our result of $25 \%$. For the LR type and $1550 \mathrm{~nm}$ laser the model predicts $35 \%$ absorption compared to our results of $31 \%$.

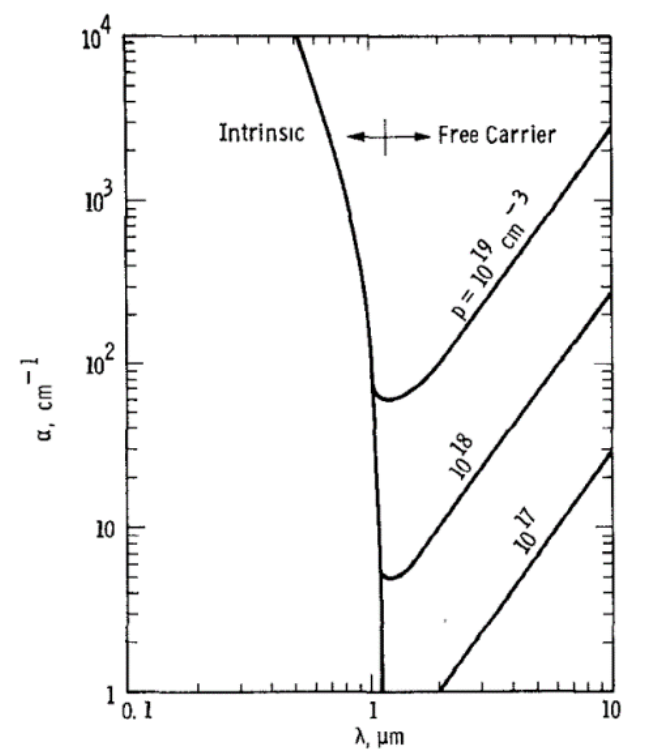

Figure 3.8 - IBA and FCA of Silicon, D. K. Schroder, IEEE 1978 [19]

Front and back side laser injection methods were explored in this chapter, and bulk $\mathrm{Si}$ resistivity and its effect on free carrier absorption were discussed here. It will be shown later in chapter 6 how the bulk resistivity effects the absorption processes in Si. A measurement of laser absorption was made for the two types of samples here, and the values were compared to a model based on Baker-Finch theory of free carrier absorption. 


\section{Chapter 4: Experimental Methods}

Our three different laser tools used for this work will be presented in this chapter. A developed method to place the thinned samples in the path of laser beam using pin grid array packages is presented here. A method used for temperature control of samples will be reviewed, and finally the design of the implemented testchip will be discussed.

\subsection{Laser Tools}

\subsubsection{An 8 Picosecond Nd:YAG Laser}

A re-purposed Laser-Voltage-Probe tool was used in our experiments. This laser tool is a dynamic waveform acquisition and analysis instrument utilizing a focused infrared laser and liquid immersion objective (LIO). LIO is a technique used to increase the resolving

power. This is achieved by immersing both the objective lens and the specimen in a transparent oil of high refractive index, thereby increasing the numerical aperture of the objective lens. The infrared $1064 \mathrm{~nm} \mathrm{Nd:YAG} \mathrm{(neodymium-doped} \mathrm{yttrium} \mathrm{aluminum}$ garnet) laser is highly stable and has a large tuning range allowing for discrete laser energies. The laser is mode-locked with automatic cavity length adjustment to allow for a very short, low-noise laser pulse generation of $8 \mathrm{ps}$. This laser system has two embedded solid state lasers that emit laser radiation at $1064 \mathrm{~nm}$ wavelength. One laser is a modelocked laser emitting 7 - 10ps wide pulses at $100 \mathrm{MHz}$ repetition frequency. It has a maximum average output power of $<450 \mathrm{~mW}$ and maximum pulse energy of $<4.5 \mathrm{~nJ}$ directly out of the laser head. The second laser is a laser diode that emits $<50 \mathrm{~mW}$ of average power. It operates in both pulsed mode and continuous wave $(\mathrm{CW})$ mode, depending on 
the state of the system (imaging or waveform acquisition). In pulsed mode, the laser diode pulse width is nominally $1.3-2.0 \mu$ s with peak power $<50 \mathrm{~mW}$ and pulse energy $<0.1 \mu \mathrm{J}$. Both embedded Class $3 b$ lasers are located inside the laser rack. Radiation from these lasers is delivered to the acquisition area using metal-jacketed fiber optic cables.

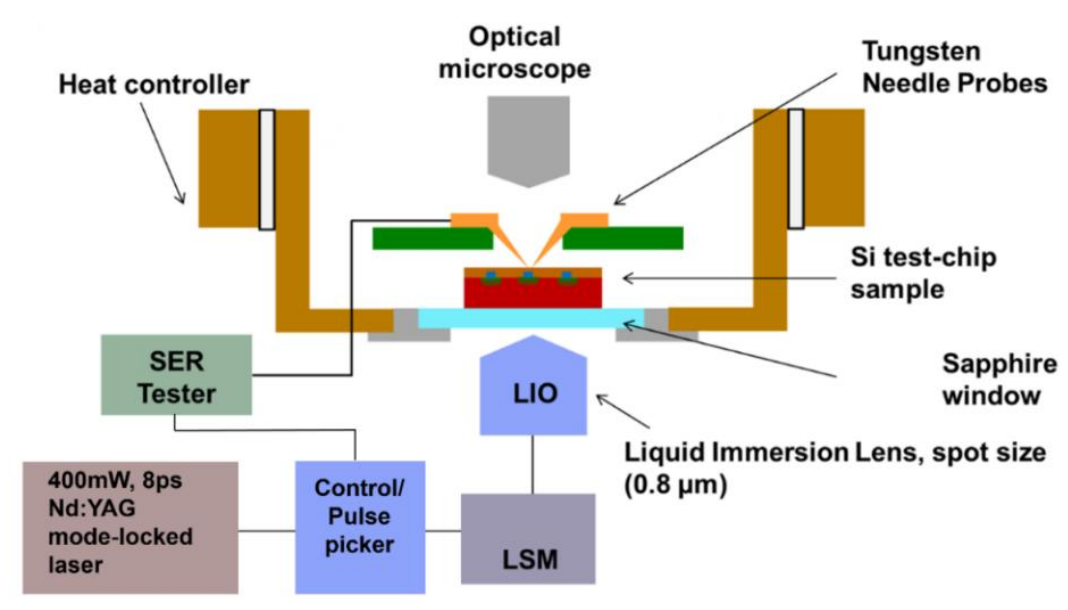

Figure 4.1 - A diagram of Nd:YAG laser apparatus

Figure 4.1 is a diagram of the laser system and our testing apparatus. All temperature related data were collected using this tool. Laser pulses with known energy values come from the bottom, and hit the backside of the testchip under study. Thinned samples are placed on top of a layer of sapphire. A clear hydrocarbon-based optical fluid with refractive index similar to sapphire is placed under the sample for better visibility and heat conduction. A tungsten needle probecard specific to the sample under study is mounted on a XYZ translation stage. The probecard is landed on the sample for data acquisition. The numerical aperture of the objective is 1.4 , producing a spot size of 0.76 micrometer.

\subsubsection{A 40 femtosecond Yb:YAG laser}


Single photon absorption experiments were conducted using an Ytterbium-based (ytterbium-doped yttrium-aluminum garnet) solid-state femtosecond laser. This tool is a mode-lock laser. Mode-locking allows for simple, self-starting passive mode-locking of ultrafast solid-state lasers that are capable of generating short pulses with a simple configuration. This laser emits infrared light at $1040 \mathrm{~nm}$, and delivers high peak power and ultrashort pulses, while maintaining long-term stability. It has a minimum output power of $2.5 \mathrm{~W}$ and peak power of 20MW. Figure 4.2 is a diagram of our experiment setup. Laser beam passes through a pump-probe setup as shown in figure 4.3, and enters an objective lens where it is refocused before targeting the desired location on the sample testchip.

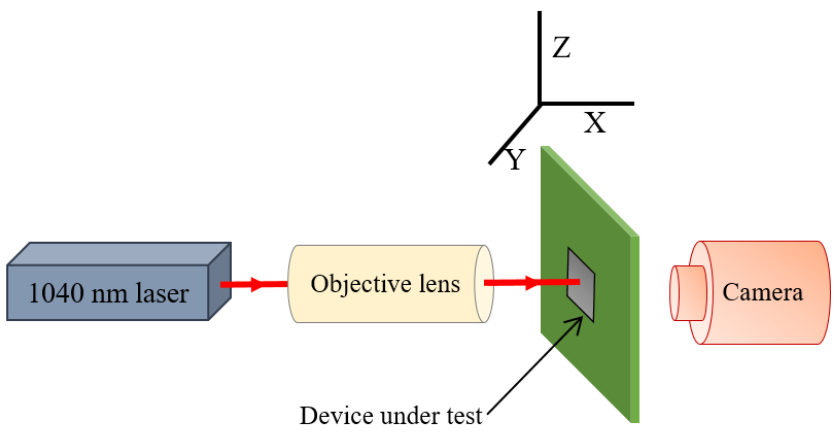

(a)

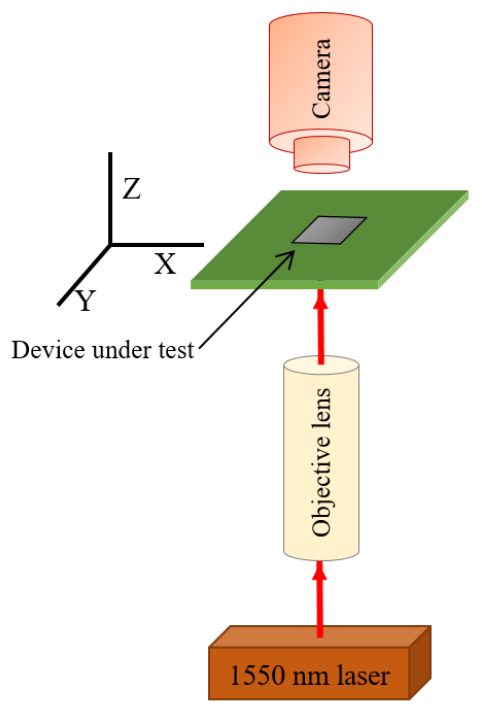

(b)

Figure 4.2 - $1040 \mathrm{~nm}$ (a) and $1550 \mathrm{~nm}$ (b) laser experiment setups. They both setups sample can move along a plane perpendicular to laser beam.

Unlike the laser tool discussed in 4.1.1, in this setup laser is kept stationary. Testchip is mounted on a XYZ translation stage with manual high precision motion control. 
Figure 4.3 is a top view of the pump-probe apparatus. The purpose of pump-probe setup is to verify two-photon absorption effect, and is unrelated to SPA experiment. Here we merely used the pump beam containing $95 \%$ of the beam intensity for our work. This laser has a numerical aperture of 0.8 giving a laser spot size of 1.3 micrometer.

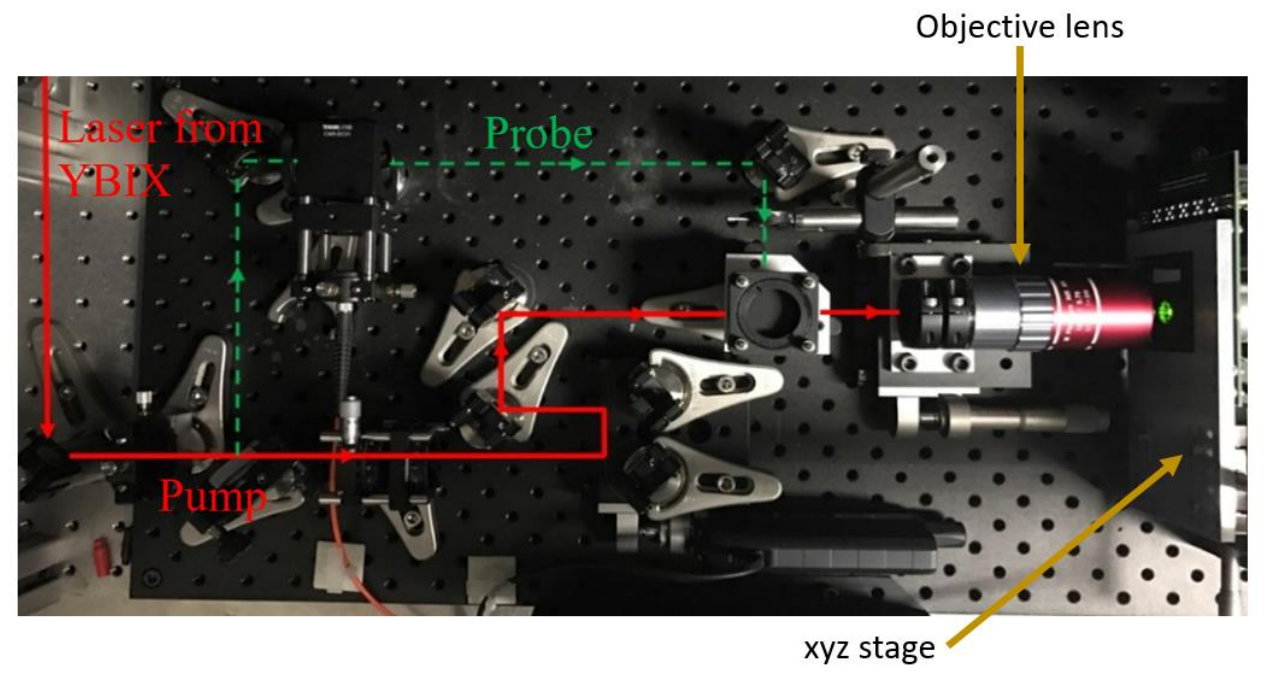

Figure 4.3 - Pump-probe setup. Red path indicates the laser beam used in SPA experiment

\subsubsection{A compact fiber based femtosecond laser}

Two photon absorption experiments were conducted using this laser. This femtosecond fiber laser provides high power, ultrashort pulses, and high levels of stability. It is an aircooled, $1550 \mathrm{~nm}$ fiber-based femtosecond laser with output powers from 0.2 to greater than $1.0 \mathrm{~W}$, with pulse widths of less than $90 \mathrm{fs}$. The building block of the laser is an ultrafast fiber laser seed platform, which utilizes a passive mode-locking technology. This tool has a numerical aperture of 0.8 and is capable of producing a spot size of around 2 micrometers. The experiment setup for both SPA and TPA is demonstrated in figure 4.2. Laser beam was located along z-axis targeting the sample from the bottom. Laser had a stationary 
position, and the sample was placed on an xyz translation stage. A pair of polarizers in the path of the laser beam were used to control the power reaching the sample.

\subsection{Glass-bottom PGA method}

When we first started this work, we had to place the testchip on a sapphire window with a drop of optical oil in between the two layers. We would then land the probecard on the sample to start. One of the difficulties in gathering data this way was the lack of stability especially at higher temperatures. To achieve better stability and more reliable results, testchips were mounted on pin grid array (PGA) packages as shown in figure 4.4. Samples were attached to a thin layer of sapphire using a UV activated glue with similar refractive index to sapphire. The sapphire was then attached to the PGA package using the same glue. Samples were then wire bonded to the PGA package ready for testing. We used a standard PGA socket as shown in figure 4.4 to connect the package to the board.

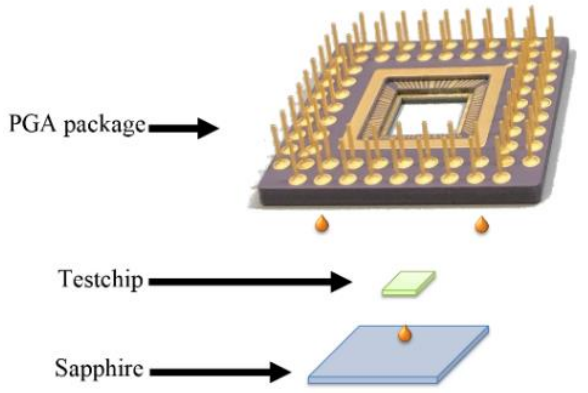

(a)

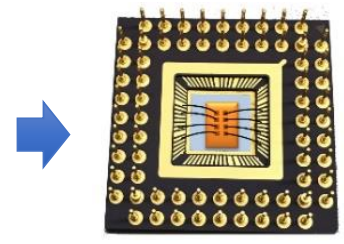

(b)

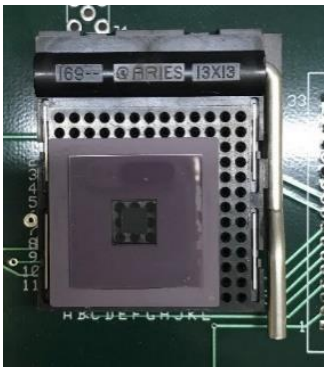

(c)

Figure 4.4- Sapphire bottom PGA Sample preparation 


\subsection{Heat Control}

Temperature variability of samples is accomplished using thermoelectric heating devices also called Peltier heat controllers mounted on a metal plate under the samples. Thermoelectric cooling (TEC) has become the method of choice for fast and compact temperature control. An electrical current through a Peltier element produces an active heat transport. With one side attached to a heat sink, the 'object' side of the thermoelectric element can be heated with respect to the sink. Important characteristics of a Peltier controller are its precision and stability, safety and efficiency. In a Peltier element, $\mathrm{N}$ and P type semiconductors, electrically connected in series, are placed in parallel to each other and are sandwiched within a pair of thermally conducting plates.

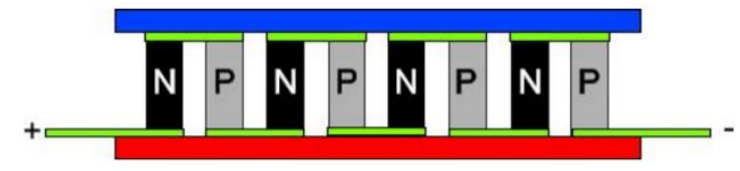

Figure 4.5 - Diagram of Peltier heater

When a voltage is applied across the device, heat is transferred from one side to the other, causing one side to get cooler while the other gets hotter, with consumption of electrical energy (figure 4.5). A thermistor is installed on the metal plate in touch with heater, holding the sample that continuously reports the device temperature. A feedback loop is programmed to maintain constant temperatures.

\subsection{Implemented Testchip}

The testchip used to collect data is designed by B. Gill et al. of Intel. Our test-chip contained NMOS and PMOS devices with a master-slave flip-flop configuration. It contains 
thousands of instantiations of several flavors of sequential elements each, all chained together in a shift register fashion. Each cell is a static storage element, consisting of a pair of cross-coupled inverters. In each inverter, the OFF-device's diffusion [drain] is vulnerable to collecting ionizing-particle-induced charge that can disrupt the stored state. The ON device's diffusion, on the other hand, collects charge that reinforces the stored data. [21] Test-chips in off state were injected with pulsed laser.

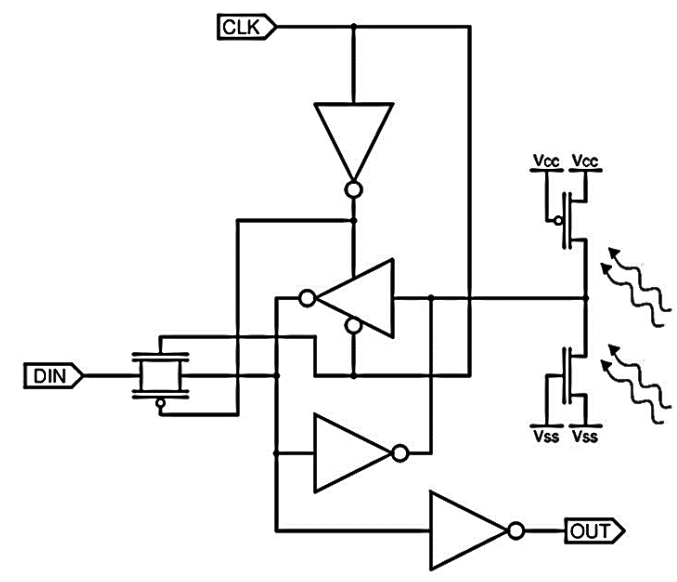

Figure 4.6- Single event upset on flip-flop

Our laser tools, and the experimental setup used for each tool was reviewed in this chapter. A novel method to use PGA packaging for the thinned samples was introduced. The simplicity of the preparation procedure and practicality of implementation of the PGA packaging makes this method extremely valuable. Most of our data presented in this work was taken using the PGA method. 


\section{Chapter 5: Laser Overview}

In this chapter I will review some basic background regarding lasers. I will then summarize the nonlinear optical effects such an intensity-dependent refractive index, that are relevant to this work. An overview of two-photon absorption process will then be presented. Finally, I will demonstrate our method of verifying TPA.

\subsection{Optics Background}

Every laser system has a gain medium, placed between a pair of optically parallel and highly reflecting mirrors with one of them partially transmitting, and an energy source to pump the gain medium. After attaining considerable amplification light emits through the transmitting mirror. [22] A Gaussian beam is a radially symmetrical distribution of monochromatic electromagnetic radiation whose transverse electric field is given by the Gaussian function. This transverse Gaussian mode or TEM00 mode describes the output of most lasers, as such a beam can be focused into the most concentrated spot. The electric and magnetic field amplitudes of any Gaussian beam are determined by a parameter called waist $w_{0}$. The waist is the radius of the beam at focus where beam radius $w(z)$ is minimum, and intensity is at maximum. The electric field of this beam is given by

$$
E=E_{0} \exp \left(\frac{-r^{2}}{w(z)^{2}}\right)
$$

where $E_{0}$ is the initial magnitude of electric field, $w(z)$ is the radius at which the intensity is $1 / e^{2}$ of its peak value, and $\mathrm{r}$ is the radial distance from the axis. The intensity distribution of the beam is 


$$
I=I_{0}\left(\frac{w_{0}}{w(z)}\right)^{2} \exp \left(\frac{-2 r^{2}}{w(z)^{2}}\right)
$$

where $I_{0}$ is the intensity at the center of the beam. The Rayleigh length is the distance from the beam waist to where the beam radius is increased by $\sqrt{2}$. The Rayleigh length given by

$$
z_{R}=\frac{\pi w_{0}^{2}}{\lambda}
$$

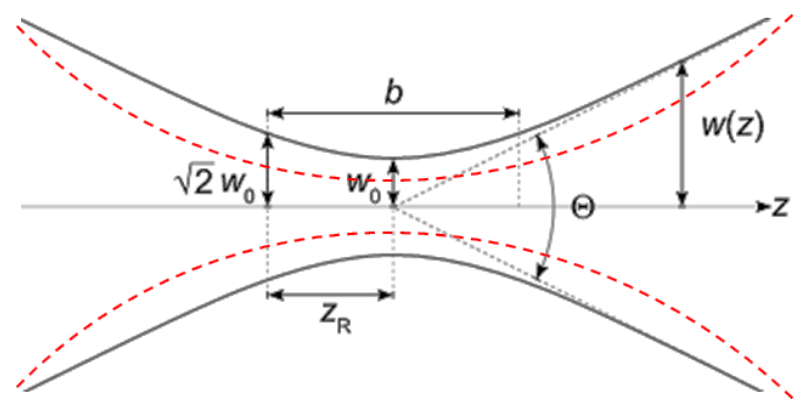

Figure 5.1 - Laser waist and Rayleigh length

Even though the tail of a Gaussian function never actually reaches zero, here I will consider the beam to have an edge at the radius $r=w(z)$, where the intensity has dropped to $1 / e^{2}$ of its maximum value. For $z \ll z_{R}, w(z)$ increases linearly with $z$. This means that far from the waist, the beam is cone-shaped. The angle between lines along that cone and the central axis of the beam is called the beam divergence, and it is given by

$$
\theta=\frac{\Theta}{2} \cong \frac{\lambda}{\pi w_{0}}
$$

Numerical aperture, $N A$, of any optical system is $N A=n \sin \theta$, and defines the range of angles over which the system can accept or emit light. [23] [24] Because the divergence is inversely proportional to the spot size, for a given wavelength, a Gaussian beam that is focused to a small spot diverges rapidly as it propagates away from the focus. The 
resolution of a given instrument is proportional to the size of its objective. When a laser beam focus is at maximum and the divergence is at minimum for the given wavelength, it is known to be diffraction-limited. Diffraction limit is described by

$$
\theta_{\text {diffraction }}=\frac{1.22 \lambda}{D}
$$

where $\mathrm{D}$ is the lens diameter. At high laser intensities, the waist is expected to decrease drastically and results in a much finer focus spot. This allows us to more accurately target the desired device. Table 3 compares the effects of SPA and TPA on focus and Rayleigh length. It can be seen that with TPA better focus can be obtained.

\begin{tabular}{|l|c|c|}
\hline & $w_{0}(m)$ & $z_{R}(m)$ \\
\hline SPA & $3.2 \times 10^{-6}$ & $3.4 \times 10^{-6}$ \\
\hline TPA & $1.8 \times 10^{-6}$ & $2.0 \times 10^{-6}$ \\
\hline
\end{tabular}

Table 3 - Focus at SPA and TPA

\subsection{Femtosecond Lasers}

Femtosecond (fs) light pulses are electromagnetic wave packets and as such are fully described by the time and space dependent electric field. In the frame of a semi-classical treatment the propagation of such fields and the interaction with matter are governed by Maxwell's equations with the material response given by a macroscopic polarization. Following the advancements in optics, the field of ultrafast optics emerged from modelocking studies of solid-state and organic dye lasers in the 1960s and 1970s. The discovery of self-mode-locking in a Ti: sapphire laser is marked as a starting point that triggered the progress in the generation of femtosecond pulses with solid state lasers. 
Short-pulse lasers are commonly used in applications such as time resolved spectroscopy and precision material processing. Recent developments in the field are directed to lasers generating higher output power and shorter pulses. [18] [19] When dealing with continuous wave $(\mathrm{CW})$ or nanosecond duration laser pulses, it is typically assumed that most of the absorption is due to single photon interactions. However, for picosecond and femtosecond lasers, the extremely high instantaneous intensity enables phenomena such as multiphoton absorption which can significantly decrease absorption depths. [25].

\subsection{Nonlinear Effects}

The response of any dielectric to light becomes nonlinear for intense electromagnetic fields. This nonlinearity is usually observed at very high laser intensities. On a fundamental level, the origin of a nonlinear response is related to anharmonic motion of the electrons under the influence of the applied field. The dipole moment per unit volume (polarization $\widetilde{P}(t)$ ) of a material depends upon the strength $\widetilde{E}(t)$ of the applied optical field. In the case of linear optics, the induced polarization depends linearly upon the electric field and can be described by the relationship

$$
\widetilde{P}(t)=\chi^{(1)} \tilde{E}(t)
$$

where $\chi^{(1)}$ is known as the linear susceptibility. In nonlinear optics, the optical response

can be described by expressing the polarization $\widetilde{P}(t)$ as a power series in the field strength $\tilde{E}(t)$ as

$$
\widetilde{P}(t)=\chi^{(1)} \tilde{E}(t)+\chi^{(2)} \tilde{E}^{2}(t)+\chi^{(3)} \tilde{E}^{3}(t)+\cdots
$$


The quantities $\chi^{(2)}$ and $\chi^{(3)}$ are known as the second- and third-order nonlinear optical susceptibilities, respectively. The linear susceptibility, $\chi^{(1)}$, represents the dominant contribution to $\widetilde{P} \cdot \chi^{(2)}$ is responsible for such effects as second harmonic generation, and $\chi^{(3)}$ is responsible for phenomena such as non-linear refractive index, four-wave mixing and two-photon absorption. Four-wave mixing is the coherent interaction of two or more frequencies of light in a nonlinear medium such as an optical fiber. These nonlinear effects in optical fibers can be observed at relatively low power levels. This is mainly due to small spot size and extremely low loss. [26]

\subsubsection{Nonlinear Refractive Index}

When light with high intensity propagates through a nonlinear medium, it can cause a change in the refractive index proportional to the optical intensity of the light. This is also referred to as the Kerr effect. The nonlinear refractive index is usually written as

$$
n=n_{0}+n_{2} I(t)
$$

where $I(t)$ is the pulse intensity given by

$$
I=\frac{P(t)^{2}}{A_{e f f}}
$$

where $P^{2}$ is the time-dependent power associated with the pulse, and $A_{e f f}$ is the effective area of the beam in the nonlinear medium. In optics, the third order susceptibility $\chi^{(3)}$ is responsible for the Kerr effect. [23] The intensity dependence of refractive index leads to other nonlinear effects such as self-phase modulation (SPM). SPM refers to the selfinduced phase shift experienced by an optical field during its propagation in optical fibers. SPM is responsible for spectral broadening of ultrashort pulses. [26] One of the processes 
that can occur as a result of the intensity-dependent refractive index is self-focusing. This process can occur when a beam of light having a non-uniform transverse intensity distribution propagates through a material in which $n_{2}$ is positive. As a result, the laser beam induces a refractive index variation within the material with a larger refractive index at the center of the beam than at its periphery. Under these conditions, the material effectively acts as a positive lens, which causes the rays to curve toward each other. [27]

\subsubsection{Laser Self-focusing}

Self-focusing is a non-linear optical process induced by the change in refractive index of materials exposed to laser light. [27] It is responsible for a dramatic increase in the local intensity during laser propagation within the medium. Because of the intensity dependence of the refractive index (equation 25), the peak of the beam experiences a larger refractive index than the wings of the profile due to the higher intensity. Therefore, the central part travels at a slower phase velocity than the edges and the wavefront of the beam is distorted in a similar fashion to a focusing lens [28]. The peak intensity of the self-focused region keeps increasing as the wave travels through the medium, until defocusing effects or medium damage interrupt this process. Self-focusing of the beam is limited by diffraction, and depending on which process dominates, a beam with finite diameter will either diverge, focus further or propagate with a constant diameter. [28] The power, not the intensity, of the laser beam is crucial in determining if self-focusing will occur. When the input power reaches a threshold value, the self-focusing balances diffraction and the beam propagates with a constant diameter. [24]

\subsubsection{Two-Photon Absorption}


In the process of two-photon absorption (TPA), an atom makes a transition from its ground state to an excited state by the simultaneous absorption of two laser photons (Figure 5.2). In the presence of TPA the equation for light propagation can be written as

$$
\frac{d I(z)}{d z}=-\alpha I(z)-\beta I(z)^{2}
$$

where $I(z)$ is the laser intensity propagating in the absorbing medium, and $\beta$ is the twophoton absorption coefficient. The solution to above equation is [29]

$$
I(z)=I_{0}\left[\frac{\exp (-\alpha z)}{1+\left(\frac{\beta I_{0}}{\alpha}\right)(1-\exp (-\alpha z))}\right]
$$

In conventional linear optics, the absorption cross section $\sigma$ is a constant. Consequently, the carrier transition rate $\mathrm{R}$ due to two-photon absorption scales as the square of the laser intensity, since $R=\sigma I / \hbar \omega$ or as

$$
R=\frac{\sigma^{(1)} I}{\hbar \omega}+\frac{\sigma^{(2)} I^{2}}{\hbar \omega}
$$

Where $\sigma^{(1)}$ and $\sigma^{(2)}$ are SPA and TPA absorption cross-section respectively. The response of a semiconductor under the condition $\hbar \omega<E_{g}$ is of a nonlinear nature, because the photon energy is too small to allow SPA to populate interband absorption. The two-photon absorption coefficient can be defined such that

$$
\alpha=\alpha_{0}+\beta I
$$


where $\beta$ is the two-photon absorption coefficient. At low energy densities $\beta$ is practically zero, however, at high laser intensities $\beta$ becomes comparable to the linear term, $\alpha$. [24]

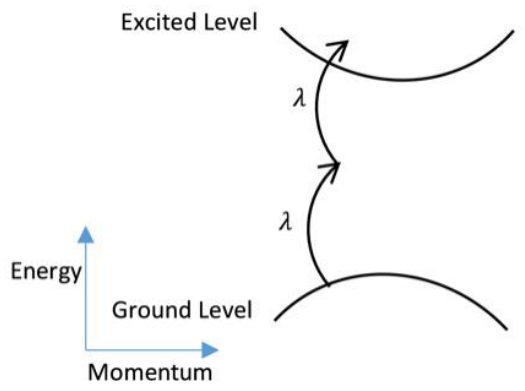

Figure 5.2 - Two-photon absorption process

\subsection{Autocorrelation}

Autocorrelation is a common method of diagnosing laser setups. It is the correlation of a signal with itself at different points in time using an interferometer apparatus figure 5.3 left). It is commonly used to estimate the duration of ultrashort pulses produced by modelocked lasers. A beam splitter splits an incoming pulse into two pulses. Each pulse will then travel a known distance through adjustable arms (Figure 5.3). The arm length difference and thus the relative timing of the pulses can be mechanically adjusted via the variable delay line. The pulses are then rejoined and sent into a crystal with a $\chi^{(2)}$ nonlinearity. If the arm length difference is made in such a way that the pulses reach the nonlinear crystal at the same instant, the process of sum frequency generation occurs, leading to an output pulse with a half the input pulse wavelength. If the incoming pulse is an infrared laser the output pulse will be green. The beam is then passed through a focusing lens, a filter which blocks out all other wavelengths except those equal to $2 \omega$. The classical 
autocorrelation setup requires a nonlinear crystal and a filter that are prohibitively expensive.

To measure the pulse duration for the $1040 \mathrm{~nm}$ laser tool we adopted a modified version of autocorrelation. In our simplified version, instead of the lens and filter, we used a light emitting diode (LED) with peak emission of $700 \mathrm{~nm}$ and with half width of $20 \mathrm{~nm}$. The following criterion had to be met in choosing the LED:

$$
1 / 2 \lambda_{\text {pulse }}<\lambda_{L E D}<\lambda_{\text {pulse }}
$$
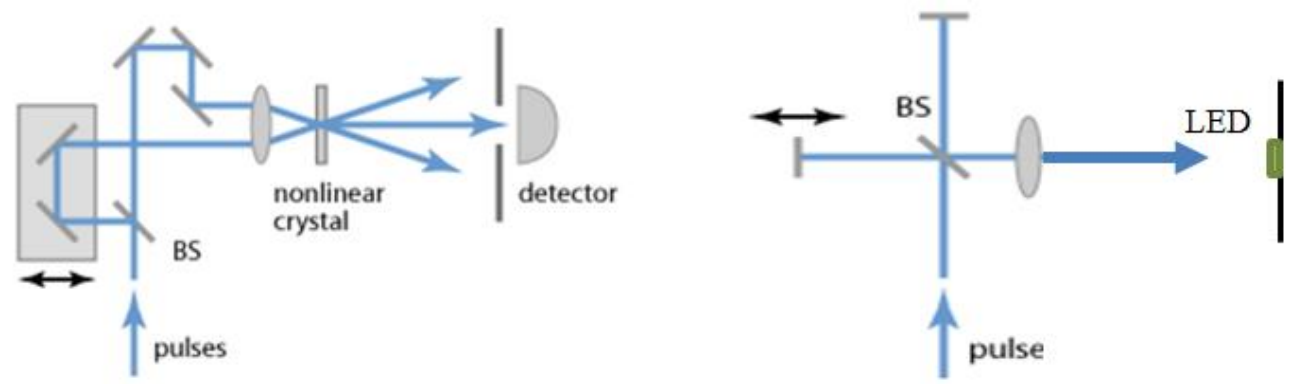

Figure 5.3 - Traditional autocorrelation (left) and our modified version setup (right)

where $\lambda_{L E D}$ is the peak emission wavelength of LED and $\lambda_{\text {pulse }}$ is the wavelength of laser. This can also be expressed as:

$$
\hbar v_{p h}<E_{B G}<2 \hbar v_{p h}
$$

where $E_{B G}$ is the bandgap of the LED and $\hbar v_{p h}$ is the photon energy. LEDs are generally designed to emit light of a certain frequency. We are reversing the functionality of the LED by targeting it with a specific wavelength and measuring the outgoing current flow. By replacing the nonlinear crystal, the detector and the filter with a simple LED we have produced an affordable autocorrelator capable of detecting a specific wavelength of light. The ultrashort 1040nm laser pulse is divided using a beam splitter, and is sent to two arms. 
It is crucial that the arms be exactly the same length, because the two pulses need to be perfectly overlapping in time and space. After rejoining and focusing the beam, the pulses are incident on the LED. With fine adjustments of the arm lengths, we were able to detect current flowing out of the LED. Figure 5.4 is the photocurrent measured in time.

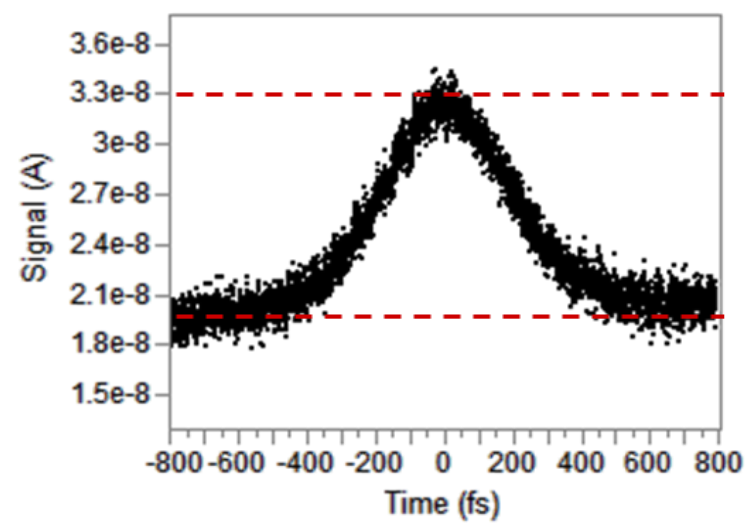

Figure 5.4 - Current signal in time for our simplified version of autocorrelation.

The LED has GaAs structure with a bandgap energy of $1.4 \mathrm{eV}$. Since the bandgap energy of the LED is large enough to not promote SPA according to equation 32, and small enough to allow TPA, detecting current after targeting the LED with the $1040 \mathrm{~nm}$ laser may be an indication of TPA. The peak in figure 5.4 corresponds to when the two arms were perfectly matching in length, thus having two wave packets at the same place and time.

Current detected is proportional to power of laser beam, and at minimum current the power of the beam is the sum of the powers of two wave packets, one from each arm of the autocorrelator (described by equation 26) $P^{2}+P^{2}=2 P^{2}$. At the peak current, the power of the beam is proportional to power of two overlapping wave packets $(2 P)^{2}=4 P^{2}$ which is the double of the beam power at minimum current. As it can be seen the current reading 
is about $18 \mathrm{nA}$ at minimum and at $33 \mathrm{nA}$ at peak, agreeing with about explanation. Therefore, we may conclude that what we detect here is the result of TPA.

Some of nonlinear effects such as self-focusing, and two-photon absorption, that are consequences of utilizing ultrafast lasers were discussed, and a variation of auto-correlation method in which a photodiode is used to detect TPA, was covered in this chapter. Advantages of laser over particle beam will be reviewed in the next chapter.

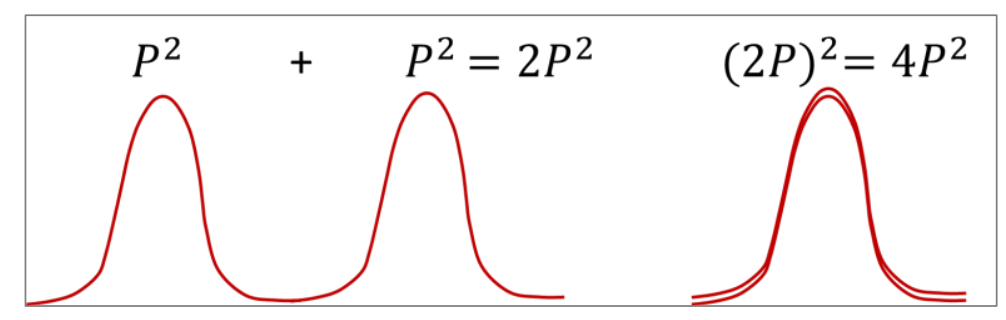

Figure 5.5 - Laser beam power at minimum (left) and at peak (right) 


\section{Chapter 6: Laser-Particle Beam Correlation}

This chapter will discuss the advantages of laser over particle beam testing. A definition of critical charge will be presented. Then we will take a closer look at beam method of defining the critical charge, and will compare that to the laser-based method. Finally, SPA results will be compared to the beam data.

\subsection{Advantages of Laser SER}

Up to the mid-90s particle accelerator testing was the standard method used to characterize the sensitivity of modern device technology to SER. However, because accelerator testing is expensive and not easily accessible, and only provides limited spatial and temporal information, other techniques have been explored. [29] Femtosecond pulsed lasers have been shown to be a reliable alternative that doesn't suffer the particle beam's limitations. [17] Figure 6.1 is the result of laser and particle beam correlation, where $\mathrm{x}$-axis presents the particle beam data cross-section and on y-axis is the laser cross-section. [17] A laser beam can generate electron-hole pairs in silicon, much like ionizing particles in an

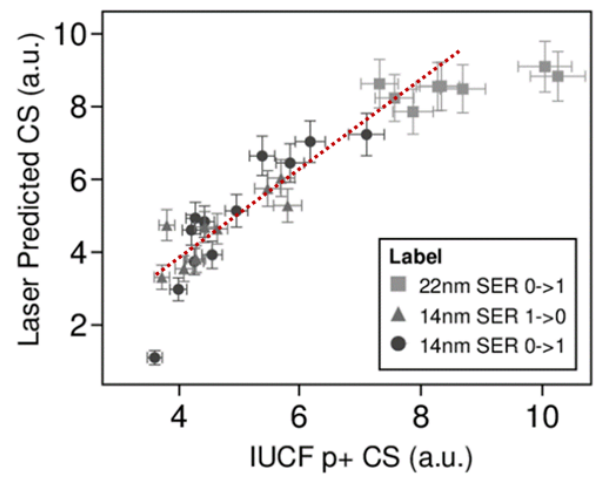

Figure 6.1 - Laser and particle beam correlation, Ascazubi IEEE 2013 [17] 
accelerator facility. The laser method offers unique control on the location and timing of the charge injection. [17] Here we validate the laser as a comparable tool for SER testing. When galactic cosmic rays reach the earth's atmosphere, they collide with atomic nuclei in air and create a chain of interactions that produce a large number of neutrons. Some of these neutrons reach the ground. Measurements of the neutron flux as a function of energy distribution has been done by Gordon et. al. (Figure 6.2). The results show a decrease in flux with neutron energy increase. In general, device sensitivity depends on the energy of the incoming particle, the geometry of the impact, the location of the strike, and the design of the logic circuit.

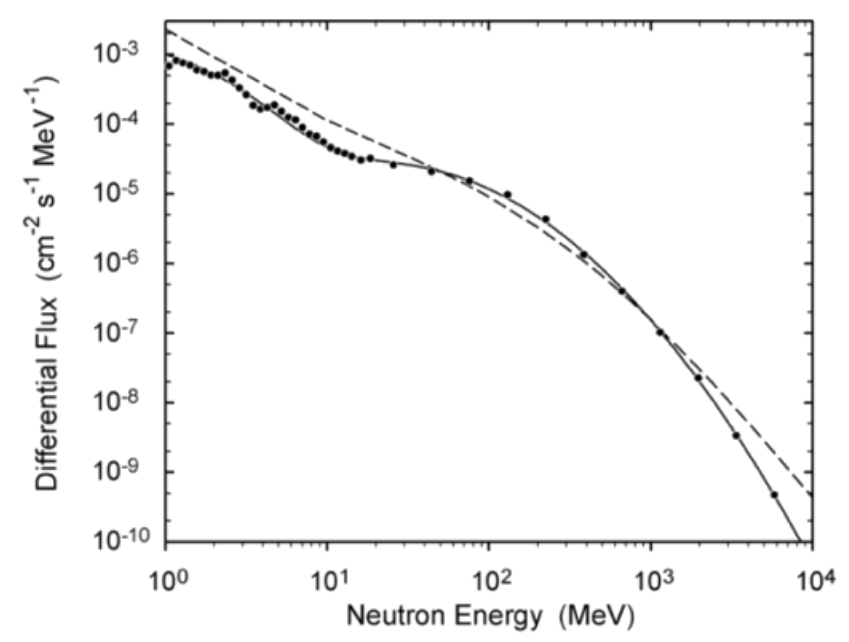

Figure 6.2 - Neutron differential flux as a function of neutron energy, Gordon IEEE 2004 [30]

\subsection{Critical Charge and Laser Cross-section}

The minimum electric charge required to cause a flip or upset is called critical charge, $Q_{c r i t}$. If the number of carriers times the charge per carrier (unit charge) is greater than $Q_{c r i t}$ we expect to detect a flip. As an example we will consider a device that experiences 
a flip with a minimum neutrons energy of $100 \mathrm{MeV}$ which is about the average neutron energy (figure 6.3).

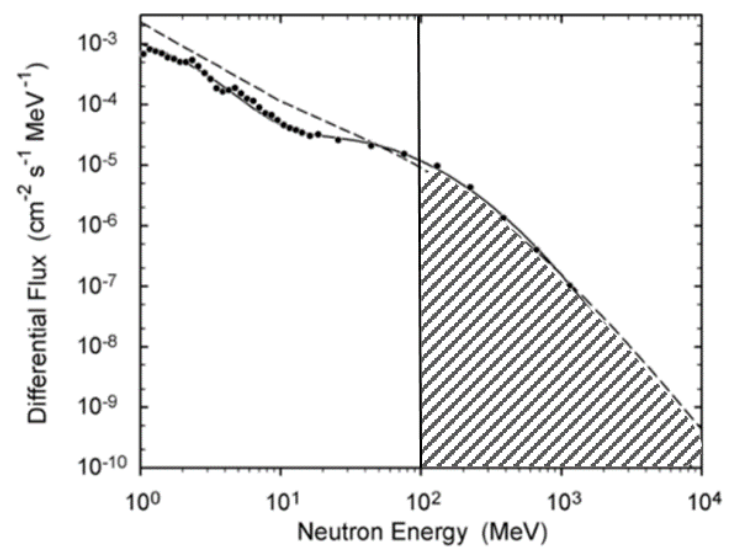

Figure 6.3 - Example of a typical energetic neutron of $100 \mathrm{MeV}$ that causes an upset in a device.

Using accelerator data, we can calculate the total $Q_{c r i t}$ created within the device. For a beam experiment

$$
n=\frac{L E T}{E_{\Gamma}}
$$

where $n$ is the total number of carriers, LET is the linear energy transfer from the energetic particle, and $E_{\Gamma}$ is the direct bandgap energy of silicon (Figure 6.3) which is $3.4 \mathrm{eV} . E_{\Gamma}$ is the vertical energy difference between the bottom of the conduction band and the top of the valence band. Here we assume that all the energy injected by the particle is going into producing electron-hole pairs. $Q_{c r i t}$ is equal to $n$ times the charge per electron. This will result in $480 \mathrm{fC}$ of $Q_{\text {crit }}$ for beam.

For a laser, let us take a typical example of a circuit upset at $0.1 \mathrm{~nJ}$ per pulse

$$
n=\eta Q E \frac{E_{p p}}{h v}
$$

where $h v$ is the bandgap of silicon, QE is quantum efficiency, and $\eta$ is the bulk absorption. 


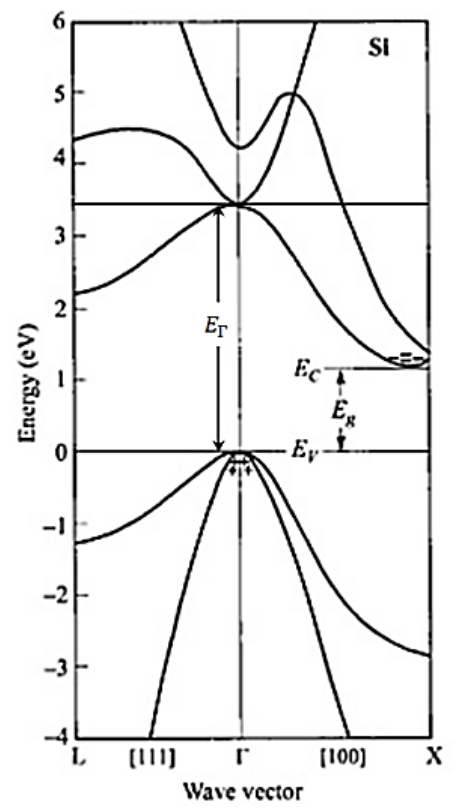

Figure 6.4 - Energy band structure of silicon - Sze p.14 2007 [35]

The QE for our laser tool is about 35\%. Using Beer's law and the relationship between laser current and power we find $\eta$ to be $2 \%$. Using the above equation $Q_{\text {crit }}$ is $636 \mathrm{fC}$. We can see that laser beam can result in same order of magnitude hit cross-section as that of the particle beam. In these calculations, we have shown the total generated charge on the region of interest is similar in both physical modes.

\begin{tabular}{|c|c|c|}
\hline & Beam & Laser \\
\hline$n$ & $3 \times 10^{6}$ & $3.97 \times 10^{6}$ \\
\hline$Q_{\text {crit }}$ & $4.8 \times 10^{-13}$ & $6.361 \times 10^{-13}$ \\
\hline
\end{tabular}

Table 4 - Comparison of charge created by particle beam and laser

Note that we are assuming that all linear energy transfer (LET) from the energetic particle has the charge has gone to creating electron-hole pairs collected by the node in question. 
Next, we will consider the cross-section of the particle beam and compare that with the laser results. Our methodology offers a scheme to predict FIT rate from estimating LET of charges generated with laser, and ultimately an alternative SER testing method to the particle beam. Generally, the relationship between laser spot and injected charge, $Q_{\text {inj }}$, can be written as

$$
\frac{Q_{i n j}}{A_{\text {spot }}}=\frac{Q_{\text {coll }}}{A_{n}}
$$

where $Q_{\text {coll }}$ is the amount of charge collected by the device, $A_{n}$ is the area of device under test and $A_{\text {spot }}$ is laser spot size. In our experiment, data was only collected when a flip happened, hence $Q_{\text {coll }}$ is equal to $Q_{c r i t}$. Then

$$
A_{n}=\frac{A_{\text {spot }} \cdot Q_{c r i t}}{Q_{i n j}}
$$

$A_{n}$ can be considered the laser induced SER cross-section.

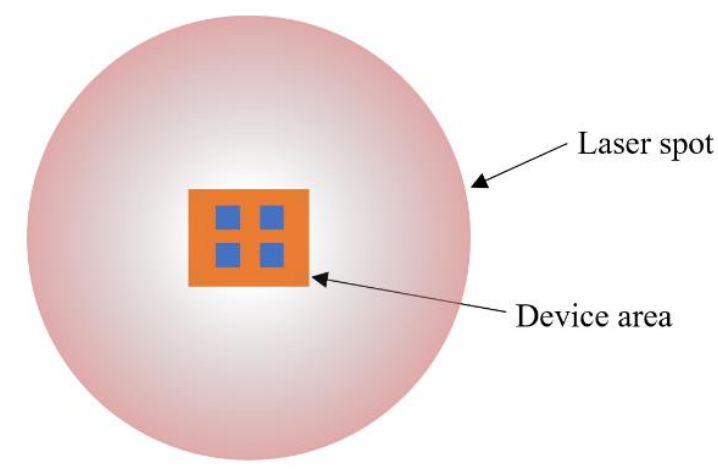

Figure 6.5 - Laser spot size compared to device area

\subsection{SPA and Particle Beam Correlation}

SPA is the linear absorption of photons whereby one photon is absorbed by an electronhole. An electron will have just about enough energy to transfer to conduction band. From 
there on the only method of transport of electron is by drift. These carriers can be regarded as cold carriers (figure 7.1). We have compared threshold energies required to cause upsets by laser to the data collected at a beam facility for the same samples. Our SPA experiment shows a reasonably good agreement between laser and particle beam (Figure 6.6).

Electron-hole pairs generated as result of a single event are subject to drift and diffusion, similar to TPA, and we expect the threshold energy values collected using the $1550 \mathrm{~nm}$ laser which will result in TPA, to more accurately present SER. Our results are presented in the next chapter.
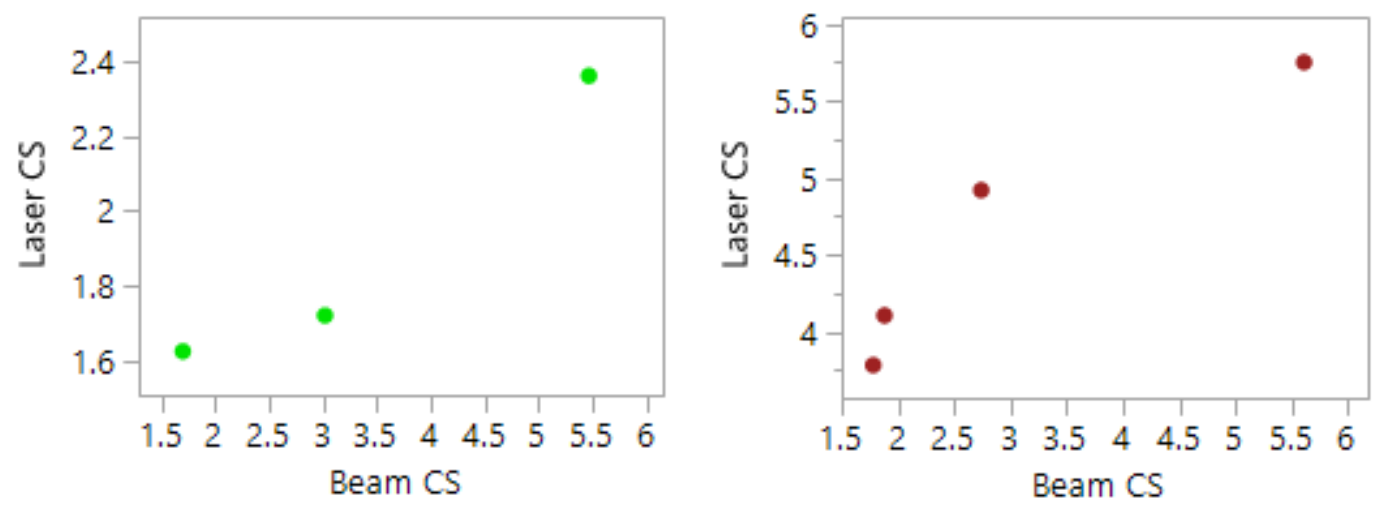

Figure 6.6 - Beam and $1040 \mathrm{~nm}$ laser correlation - NMOS (left) and PMOS (right) - result of SPA

Advantages of laser tools to study SER were considered in this chapter. While both particle accelerators and laser beams have advantages and disadvantages, they can complement each other well. The laser method offers unique control on the location and timing of the charge injection, and it proves to be a valuable resource for timely in-house testing. 


\section{Chapter 7: TPA and Relevant Effects}

This chapter will present and examine the TPA related data. TPA cross-section will be compared to beam cross-section at the end of the chapter.

TPA is a third order nonlinear optical phenomenon in which a molecule absorbs two photons at the same time. In this chapter, we will present our results from $1550 \mathrm{~nm}$ laser which leads to TPA in Si. Further we will show the different way HR and LR samples respond to TPA. The correlation between laser and beam is given at the end of the chapter. In TPA, the simultaneous absorption of two photons by one electron will cause a sudden jump of the electron from valence band to conduction band, with some leftover energy. These carriers with excess energy can be regarded as hot carriers and are subject to both drift and diffusion.

$$
J=J_{\text {drift }}+J_{\text {diffusion }}
$$

Carriers created by particle beam are also subject to both drift and diffusion, and consequently we expect the laser data of TPA to better correlate to the particle beam than SPA. Figure 7.1 is a diagram showing the comparison between SPA, TPA and particle beam energy transfer. [24]

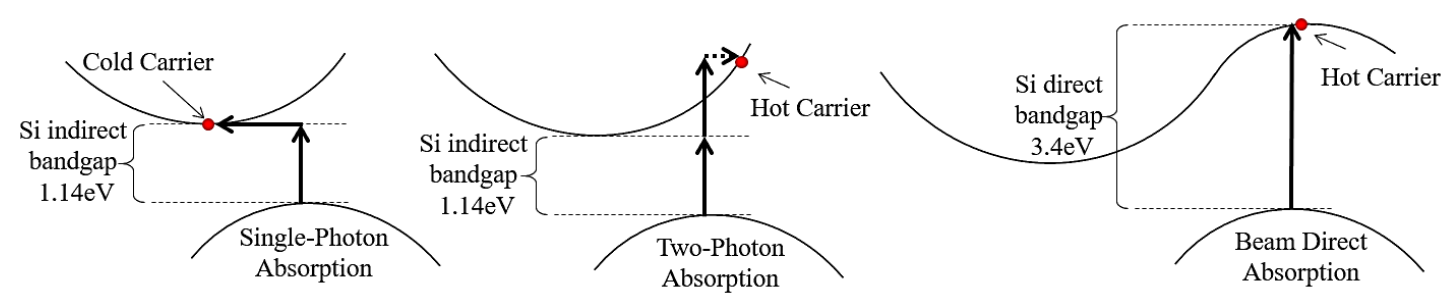

Figure 7.1 - SPA, TPA and beam energy transfer diagram 
Our $1550 \mathrm{~nm}$ ultrafast pulsed laser with $12 \mathrm{MHz}$ repetition rate was used in our two-photon experiments.

\subsection{Intensity Profile}

Two 80 micron thin samples, one LR and one HR were used to generate a profile of the beam photocurrent as a function of distance from the beam focus. To do so, we started at a position a few hundred microns above the laser beam focus and approached the focus in stepwise manner with step size of a few microns and recorded the photocurrent detected by the drain at each step using an ammeter. We used the same samples to create a beam profile for the $1040 \mathrm{~nm}$ laser as well. Figure 7.2 is the results for both lasers. In figure 7.2 location 0 refers to the current reading at beam focus

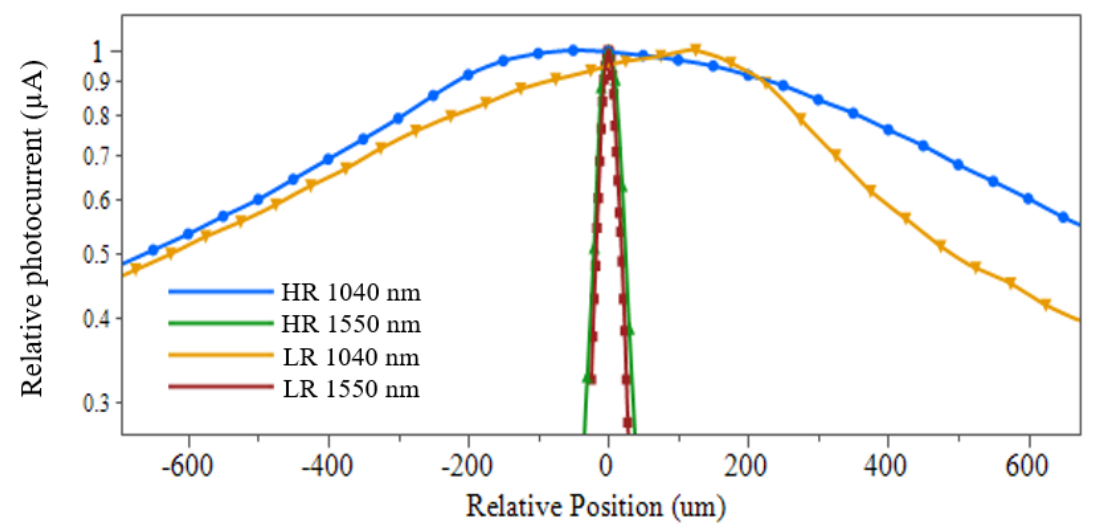

Figure $7.2-1040 \mathrm{~nm}$ and $1550 \mathrm{~nm}$ lasers intensity profile

As it can be seen, the $1550 \mathrm{~nm}$ laser has a much sharper peak. This effect can be explained if we consider the intensity profile of a free propagating Gaussian beam as shown in figure 7.2. For the $1040 \mathrm{~nm}$ laser, the intensity is a linear function of laser power,

$$
P \propto I A
$$


where $A$ is the area of laser cross-section at focus. At Rayleigh length, the laser crosssection area will be double the cross-section area of the beam at focus; if the area is doubled, the intensity will be halved of the value at the focus. At high laser intensities such as our $1550 \mathrm{~nm}$ laser, the intensity of the beam is proportional to square of the laser power (equation 26).

$$
P^{2} \propto I A
$$

In this case for the same power, if the beam cross-section area is doubled a distance equal to Rayleigh range, the intensity will be one fourth of that at the focus.

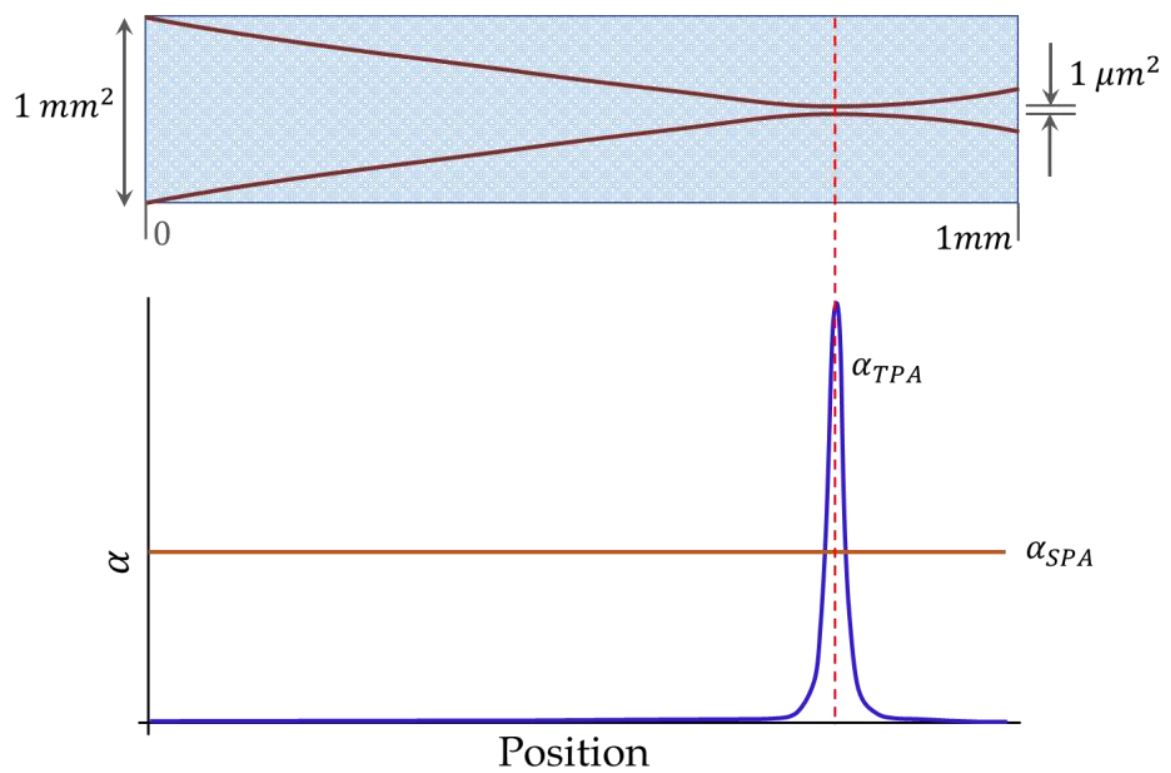

Figure 7.3 - Laser focus inside the sample at which TPA coefficient is at maximum in compare to SPA coefficient which is constant.

Figure 7.3 demonstrates this effect. TPA absorption is the method of ionization in the 1550 $\mathrm{nm}$ laser. TPA coefficient is laser intensity dependent and is at maximum at the beam focus. The $1040 \mathrm{~nm}$ laser produces ionization mainly by SPA, and SPA coefficient is constant. 


\subsection{Photocurrent and Diffusion Length}

We have collected current values for a given laser power for both type samples, at different thicknesses. Figure 7.4 shows the results.
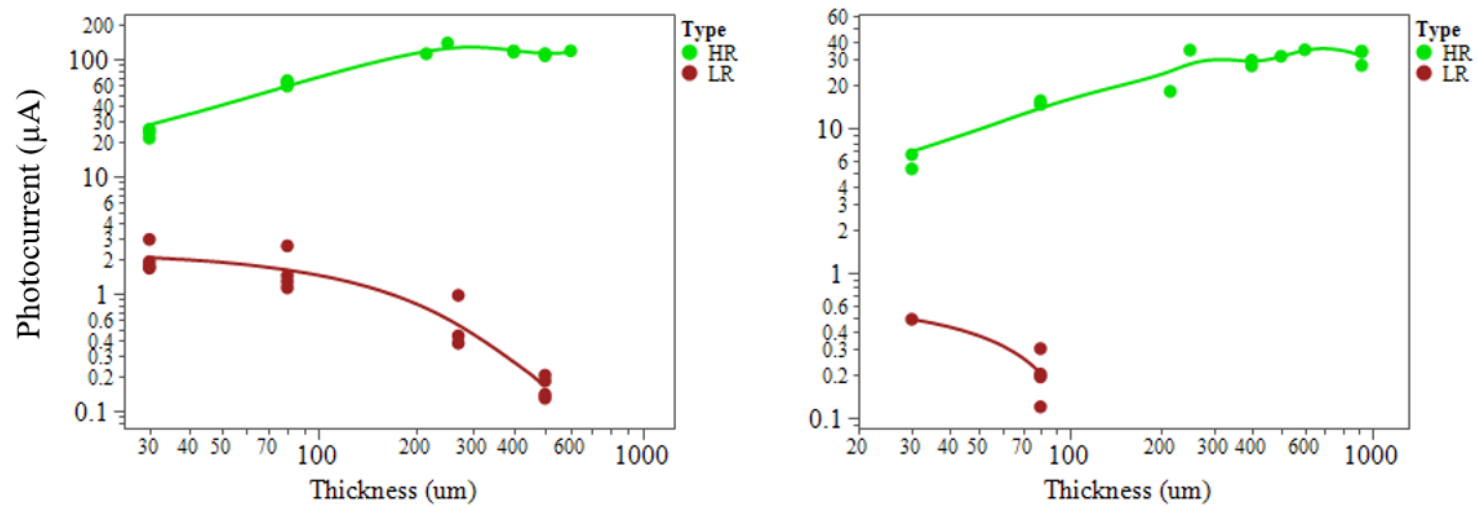

Figure 7.4 - Photocurrent vs. thickness, 1040nm (left) vs 1550nm (right)

\subsubsection{Free Carrier Absorption (FCA) vs. Interband Absorption (IBA)}

Higher current values were detected using the $1040 \mathrm{~nm}$ laser. This can be explained by considering FCA in the samples. As it can be seen from figure 3.7, FCA in HR samples is nearly zero, but the LR samples in both 1040 and $1550 \mathrm{~nm}$ experiments encounter FCA. Even though there is no IBA taking place in LR sample under 1550nm laser, FCA occurs at a much higher rate than in 1040nm laser since the absorption coefficient depends on $\lambda^{2}$.

\begin{tabular}{|c|c|c|c|c|}
\hline & Type & $\alpha_{I B A}$ & $\alpha_{F C A}$ & $\alpha_{E X P}$ \\
\hline \multirow{2}{*}{$1040 \mathrm{~nm}$} & HR & $1.8 \times 10^{3}$ & $1.74 \times 10^{-2}$ & $2.8 \times 10^{3}$ \\
\cline { 2 - 5 } & LR & $1.8 \times 10^{3}$ & $5.2 \times 10^{2}$ & $5.2 \times 10^{3}$ \\
\hline \multirow{2}{*}{$1550 \mathrm{~nm}$} & HR & 0 & $3.87 \times 10^{-2}$ & $\sim 0$ \\
\cline { 2 - 5 } & LR & 0 & $1.16 \times 10^{3}$ & $6.2 \times 10^{3}$ \\
\hline
\end{tabular}

Table 5 - Comparison of experimental and theoretical absorption coefficient 
$\alpha_{I B A}$ values were extracted from the absorption coefficient graph (figure 3.3) of section 3.1. Using Beer's law, we can calculate the percent of laser intensity absorbed by each sample. Table 1 summarizes the results. IBA and FCA in the table refer to interband absorption and free carrier absorption respectively. As it is shown $\alpha_{F C A}$ of LR with 1550 $\mathrm{nm}$ laser is about two times larger than LR with $1040 \mathrm{~nm}$ laser. Idealy

$$
I=I_{0} e^{-\alpha_{E X P} Z}=I_{0} e^{-\alpha_{I B A^{Z}}} e^{-\alpha_{F C A^{Z}}}=I_{0} e^{-z\left(\alpha_{I B A}+\alpha_{F C A}\right)}
$$

Our results for the most part agree (at least in order of magnitude) with the theory. One factor contributing to this underestimation could be coming from the calculation of $\alpha_{F C A}$. As it was discussed, $\alpha_{F C A}$ equation is based on Drude classical model. The Drude theory provides a simple theoretical framework for free carrier absorption, but possesses several deficiencies. It is derived under the condition that the semiconductor energy surface is spherically symmetric and non-degenerate; the actual band structure in $\mathrm{Si}$ is more complex. Additionally, the Drude theory is accurate only when the magnitude of the energy absorbed by free carriers is small compared with the mean carrier energy, and when the relaxation time $\tau$ is independent of energy. In practice, and in a quantum analysis, the collision interval $\tau$ depends on the nature of the scattering mechanism and the photon energy. This can result in a $\lambda$-dependence of $\alpha_{F C A}$ that varies between $\lambda^{1.5}$ and $\lambda^{3.5}$ (rather than the $\lambda^{2}$ predicted by Drude theory) in the limits of phonon or ionized impurity scattering, respectively. [11] In general, several scattering modes coexist, with the dominant mode depending on the impurity concentration and species. It is usually reasonable to expect the exponent $r$ in the dependence $\lambda^{\mathrm{r}}$ to increase with doping. [11]

\subsubsection{Penetration Depth}


It is often more sensible to think about how deep the beam penetrates beneath the sample surface. Skin depth is a measure of how deep light or any electromagnetic radiation can penetrate into a material. It is defined as the depth at which the intensity of the radiation inside the material falls to $1 / e$ or the laser power decreases to $1 / e^{2}$ of its original value just beneath the surface. [31] We can write skin depth or penetration depth as

$$
\delta=\frac{1}{\alpha}
$$

We can calculate the skin depth for our samples and 1550nm laser. For the HR sample this thickness is about $25 \mathrm{~m}$ and for LR sample it is about 160 microns. This means that if the injected laser produces a large number of electron-hole pairs in the HR sample, we can expect them to diffuse the full thickness of the sample which is about $1 \mathrm{~mm}$. Therefore, even the electron-hole pairs that were generated immediately beneath the surface could

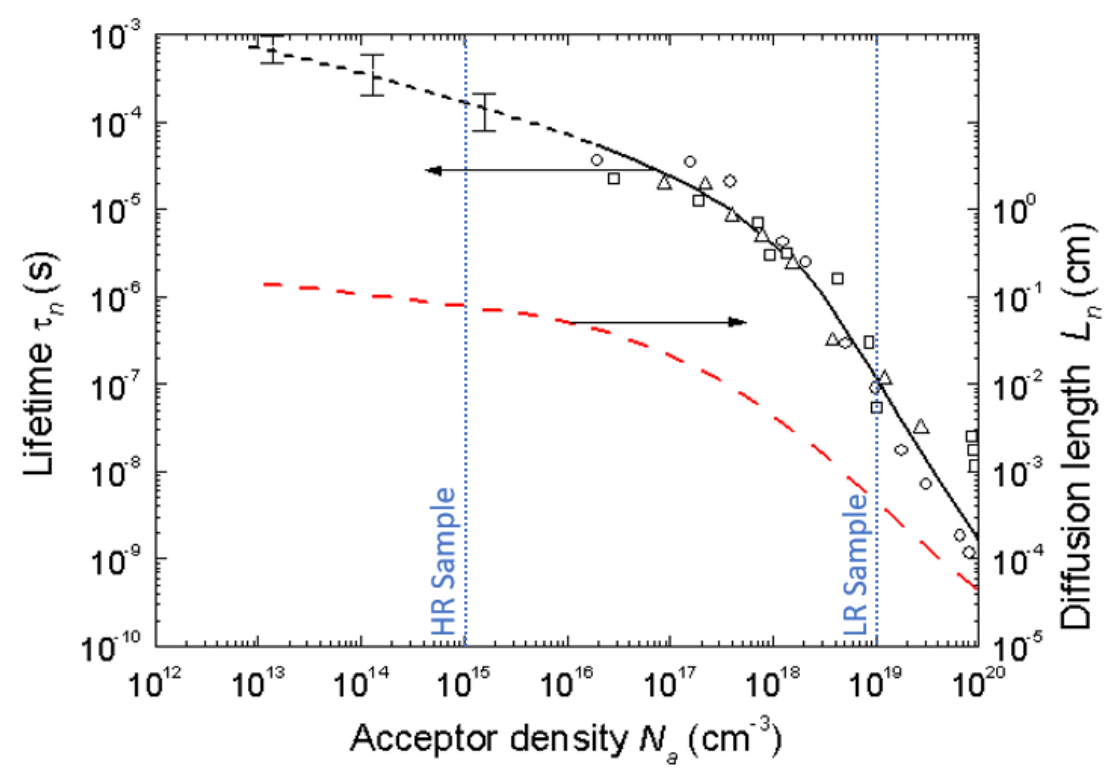

Figure 7.5 - Diffusion length and carrier lifetime as a function of carrier density - Tyagi 1983 [32] travel the whole thickness, and reach the active part of the device. For the LR samples the electron-hole pairs generated will diffuse away after about 160 microns. 


\subsubsection{Carrier Lifetime}

From figure 7.4 there is a clear difference between the amount of photocurrent detected in HR and LR samples above. Recorded current values of HR are about an order of magnitude higher than LR. This effect can be explained if we consider the carrier lifetime for the two types of sample. The bulk lifetime for extrinsic silicon can be determined using semiempirical models. The lifetime is dependent on the excess carriers and doped atoms concentrations. Diffusion length and carrier lifetime can be extracted from figure 7.5 [32]. Carrier lifetime for HR is $150 \mu \mathrm{s}$ while for LR is $0.1 \mu \mathrm{s}$. This means injected photocarriers in HR remain within the bulk $9 \times 10^{4}$ time longer than in LR samples before recombination. Similarly, the graph of figure 7.5 confirms (without any specification about wavelength) the diffusion length of LR is significantly lower than that of HR.

\subsubsection{Impact Ionization}

Another interesting result of figure 7.4 is the increase in photocurrent in HR samples with the increase in sample thickness. We believe this effect can be explained by considering the avalanche or impact ionization. At high laser intensities, a carrier gains on average more energy than it loses during collisions. Accumulating energy over time, it might overpass the ionization threshold (or the threshold of valence-conduction band excitation) of the material, and during a fortunate collision it can create a second-generation carrier. This process is called impact or avalanche ionization. The second-generation free carriers created in this manner can gain energy in the same way, and ionize other atoms or molecules. This process can be repeated as long as the field remains intense. Avalanche ionization is in fact a free carrier amplifying process. An indication for the occurrence of 
impact-ionization in MOS devices is amplifying bulk current which is what we observed. Avalanche ionization depends on the carrier velocity, and due to high carrier concentration in LR samples, carriers do not gain high enough momentum to create secondary ionization. [33]

\subsection{Soft Error Threshold Energy}

To compare our laser based SER method between the two lasers and particle beam results, we used high resistivity (HR) and low resistivity (LR) samples of different thickness in the sample setup described in section 4.1. The location of the samples was adjusted along the z-axis until they were placed at the focus of the laser beam. Similarly, xy location of the board was adjusted until the specific device was on the path of the beam. This testchip is a chain of latches designed in such way that each column of the testchip consists of different flavor of $\mathrm{N}$ or P type devices. (figure 7.6).

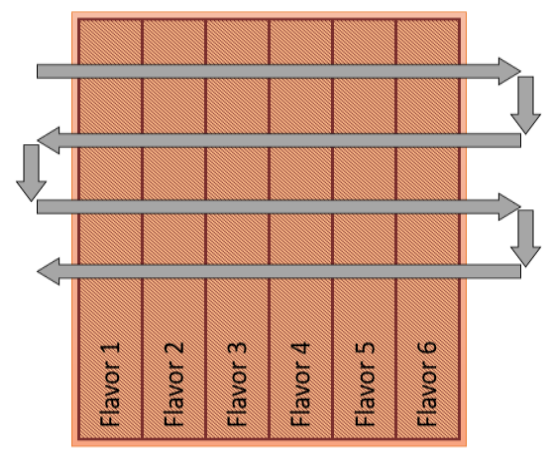

Figure 7.6 - Laser scanning pattern with respect to testchip layout

The latches are designed in a way that are increasing in source and drain area (additional fins). This does not contribute to the functionality of the chip. It only increases the crosssection area for the SER testing. To ensure that consistent exposure of all different flavors, sample was moved back and forth in front of the laser 4 times in a pattern similar to figure 
7.6. Before exposing the samples, a checkerboard pattern was written to the device, and the pattern was read back after exposure to check for logic state flips. Laser power was initially set to the lowest at which at least one logic bit was flipped, and was increased in steps of a few $\mathrm{mW}$. The experiment was performed with the same testchips utilizing both $1040 \mathrm{~nm}$ and $1550 \mathrm{~nm}$ lasers. The results will be covered in this section.

\subsubsection{Threshold Energy at Different Thicknesses}

For each thickness, the minimum laser power at which logic states were flipped $\left(E_{t h}\right)$ was extracted for both lasers. Figure 7.7 shows the results.

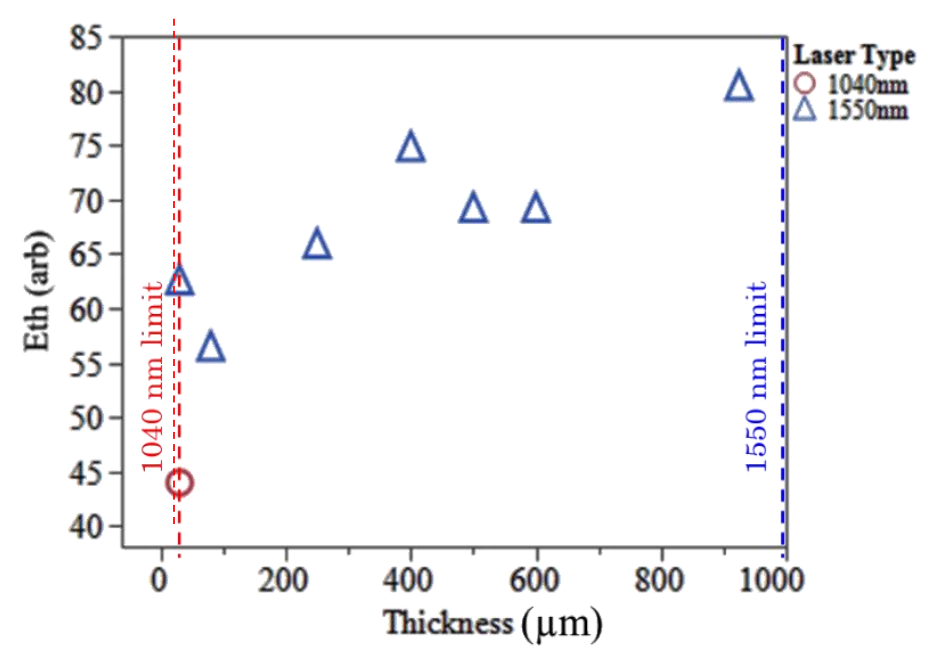

Figure 7.7 - Threshold energy vs sample thickness for HR sample using 1040nm (red) and $1550 \mathrm{~nm}$ (blue) lasers

As expected minimum energy required (Eth) to flip a bit increases with thickness. Moreover, $1040 \mathrm{~nm}$ laser cannot cause a flip in samples thicker than 80 microns, because as laser beam propagates through the sample laser energy gets absorbed through SPA. 1550 nm laser however, can get through the full thickness sample and cause flips. For the same data, we can look at the total number flips as a function of laser power. The results show that for both lasers, LR samples have more bits flipped than HR samples. Figure 7.8 shows 
the result for one of the flavors. This seems to contradict with the observation made in section 7.2.3. Even though a higher current is registered for HR samples, the total number of flips is higher for the LR samples. We believe this phenomenon can be explained by considering the nonlinear refractive index of the material. As it was described, diffusion length is significantly higher in HR, and carrier lifetime is much higher in HR than LR.

In HR, electron-hole pairs dwell in the bulk for relatively a long time before they recombine. But high carrier concentration in the bulk does not translate to more flips.

The non-linear refractive index of material in the presence of high laser intensities is dependent on the carrier concentration [27]:
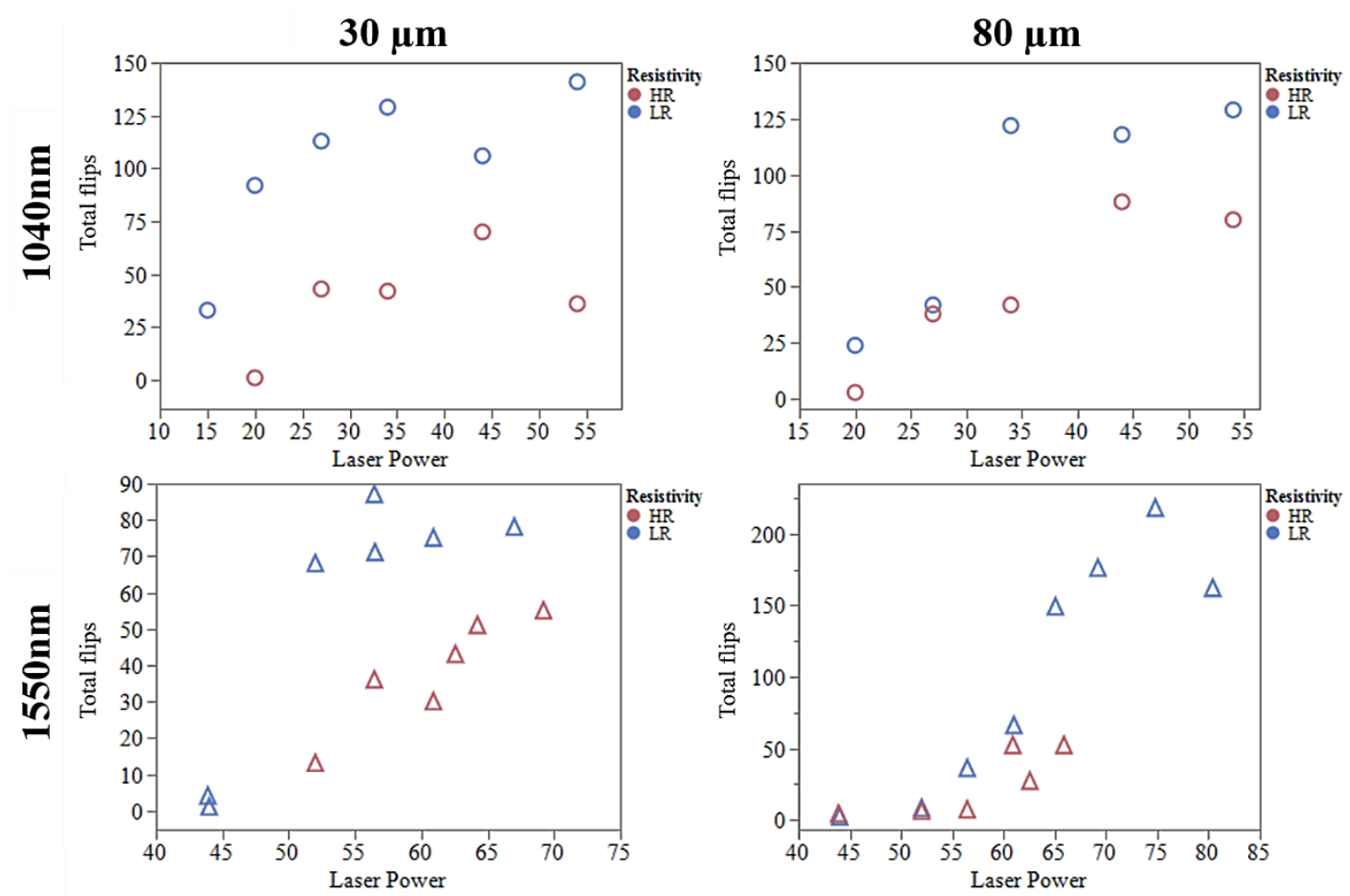

Figure 7.8 - Total number of flips as a function of laser power for $30 \mu \mathrm{m}$ (left) and $80 \mu \mathrm{m}$ (right) thick samples. The top and bottom row results are from $1040 \mathrm{~nm}$ and $1550 \mathrm{~nm}$ lasers respectively. 


$$
n=\left(n_{0}^{2}-\frac{N e^{2}}{\varepsilon_{0} m^{*} \omega_{0}^{2}}\right)^{1 / 2}
$$

We can calculate the number photocarriers, $N$, injected into the sample. To do so, we will consider the carrier concentration in a frustum cone with two circular ends, one equal to the spot size at focus and one twice as large a distance equal to Rayleigh length away (figure 7.9).

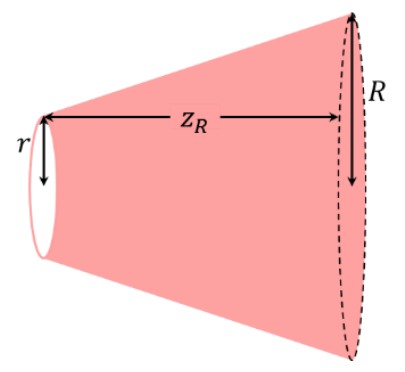

Figure 7.9 - Calculation of the volume of a frustum.

To approximately calculate photocarrier injected into the sample I will make the following assumptions:

i) I will use the minimum photocurrent recorded for the $30 \mu \mathrm{m}$ thick sample as the photocurrent generating the charge.

ii) I will make the ssumption that the total photocurrent generated is the sum of the charge generated per pulse (figure 7.10), and so

$$
O_{p p}=\frac{I_{\text {photo }}}{n}
$$


where $Q_{p p}$ is the amount of charge per pulse, $I_{p h o t o}$ is the photocurrent from (i), and $n$ is the number of pulses or the repetition rate of the laser. For the 1550 $\mathrm{nm}$ laser this is $12 \mathrm{MHz}$.

iii) The laser spot size at focus can be calculated as follows:

$$
\text { spot diameter }=1.22 \frac{\lambda}{N A}
$$

I will assume the height of the cone is equal to Rayleigh length. From figure 7.2 full width at half maximum (FWHM) of the laser intensity for $1550 \mathrm{~nm}$ is about $20 \mu \mathrm{m}$, hence taking Rayleigh length as the height of the injection volume is a valid assuption.

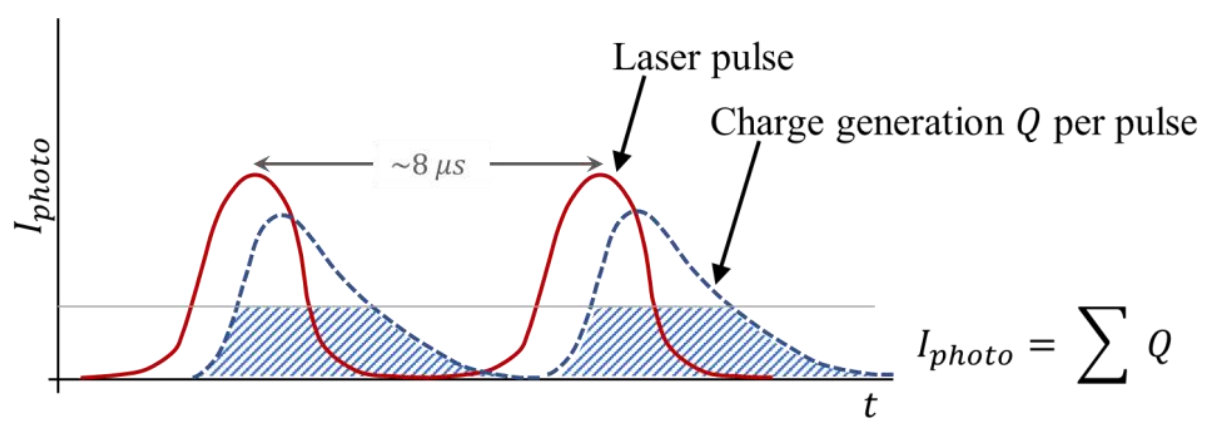

Figure 7.10 - Demonstration of charge generation following each laser pulse.

From figure $7.4 I_{\text {photo }}$ for the $30 \mu \mathrm{m}$ thick sample is about $6 \mu \mathrm{A}$. Using equation 44 the amount of charge generated per pulse can be calculated:

$$
Q_{p p}=\frac{I_{\text {photo }}}{n}=\frac{6 \times 10^{-6}}{12 \times 10^{6}}=5 \times 10^{-13} \mathrm{C}
$$

The volume of the frustum cone is equal to

$$
V=\frac{1}{3} \pi\left(R^{2}+r^{2}+R r\right) z_{R}
$$


Where $V$ is the volume of frustum, $r$ is the radius of the beam at focus, $R$ is the radius of the beam at Rayleigh length $(R=\sqrt{2} r)$ and $z_{R}$ is the Rayleigh length. For our $1550 \mathrm{~nm}$ laser the spot diameter is $2.4 \mu \mathrm{m}$. Using equation 21 Rayleigh length, $z_{R}$, for the laser can be calculated and it is about $3 \mu \mathrm{m}$. We can calculate the volume of the frustum cone:

$$
V=\frac{2+\sqrt{2}}{3} \pi r^{2} z_{R}=1.3 \times 10^{-17} \mathrm{~m}^{3}
$$

Number of carriers injected in this volume by each laser pulse can be calculated:

$$
N=\frac{Q_{p p}}{q \times \text { Volume }}=2.4 \times 10^{23} \mathrm{~m}^{-3}=2.4 \times 10^{17} \mathrm{~cm}^{-3}
$$

where $q$ is the charge per carrier $\left(q=1.602 \times 10^{-19}\right)$.
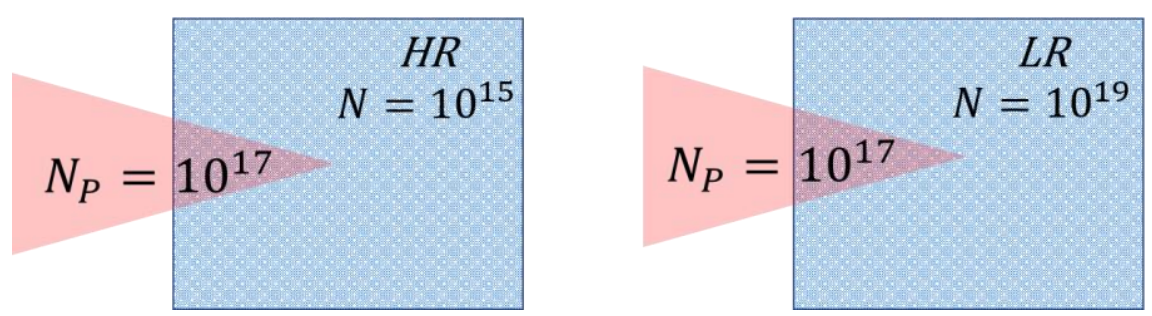

Figure 7.11 - A diagram of LR vs. HR as the laser beam penetrates

The number carriers injected to the sample is in the order of $10^{17} \mathrm{~cm}^{-3}$. For the HR sample light goes from an area with refractive index, $\mathrm{n}$ that depends on $10^{17}$ carriers to an area with $\mathrm{n}$ that depends on $10^{15}$ carriers. This change in $\mathrm{n}$ acts as a defocusing lens making the beam out of focus. For the LR sample on the other hand, light goes from an area with refractive index depending on $10^{17}+10^{19}$ carriers to an area with refractive index depending on $10^{19}$ carriers. The change in refractive index here is about 100 times smaller 
than in the HR sample, and therefore the defocusing effect is much weaker. A closer inspection of the high and low resistivity samples profile confirms this finding and reveals that LR sample has a narrower intensity profile compared to the HR. Note that according to equation 41 this is a small effect as it can be seen in figure 7.12.
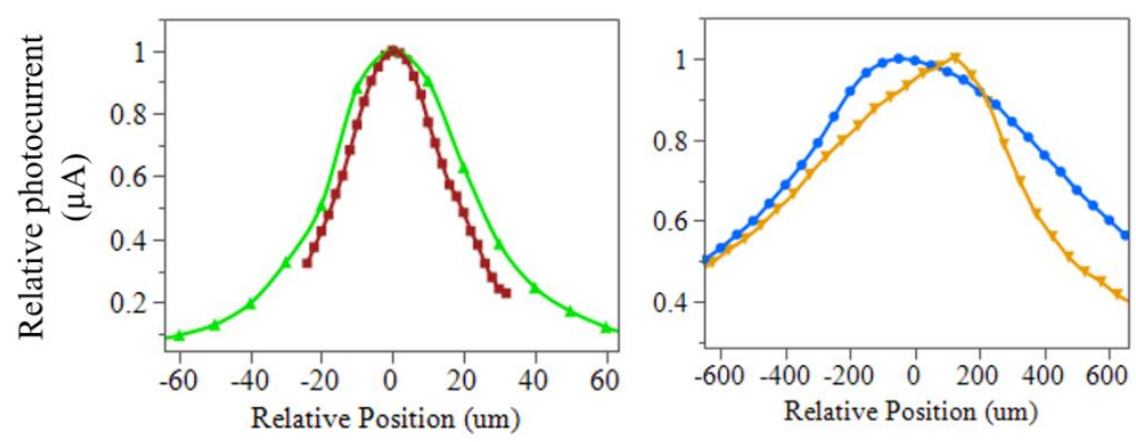

Figure 7.12 - Comparison between HR (green and blue) to LR (red and orange) intensities

\subsubsection{Laser and Beam Correlation}

We had predicted that with $1550 \mathrm{~nm}$ laser will produce better results than $1040 \mathrm{~nm}$ and would be a comparable method to particle beam. Figure 7.13 shows the correlation between our two laser tools and the beam. Even though the $R^{2}$ from figure 7.13 for $1550 \mathrm{~nm}$ laser is lower than that of $1040 \mathrm{~nm}$ laser, the close proximity of the two demonstrate that we are on the right track, with the great advantage of not having to thin the sample.

By implementing this method, we made the discovery that the HR beam is defocusing, and even though we recorded a high amount photocurrent that reaches the metal layer, it is not focused enough to cause as many flips as in LR sample. This effect could become problematic in certain samples. One improvement that could lead to better results is to 
automate the scanning procedure, and by doing so we can expect to have more consistent laser exposure that can reach more precise locations on the testchip.
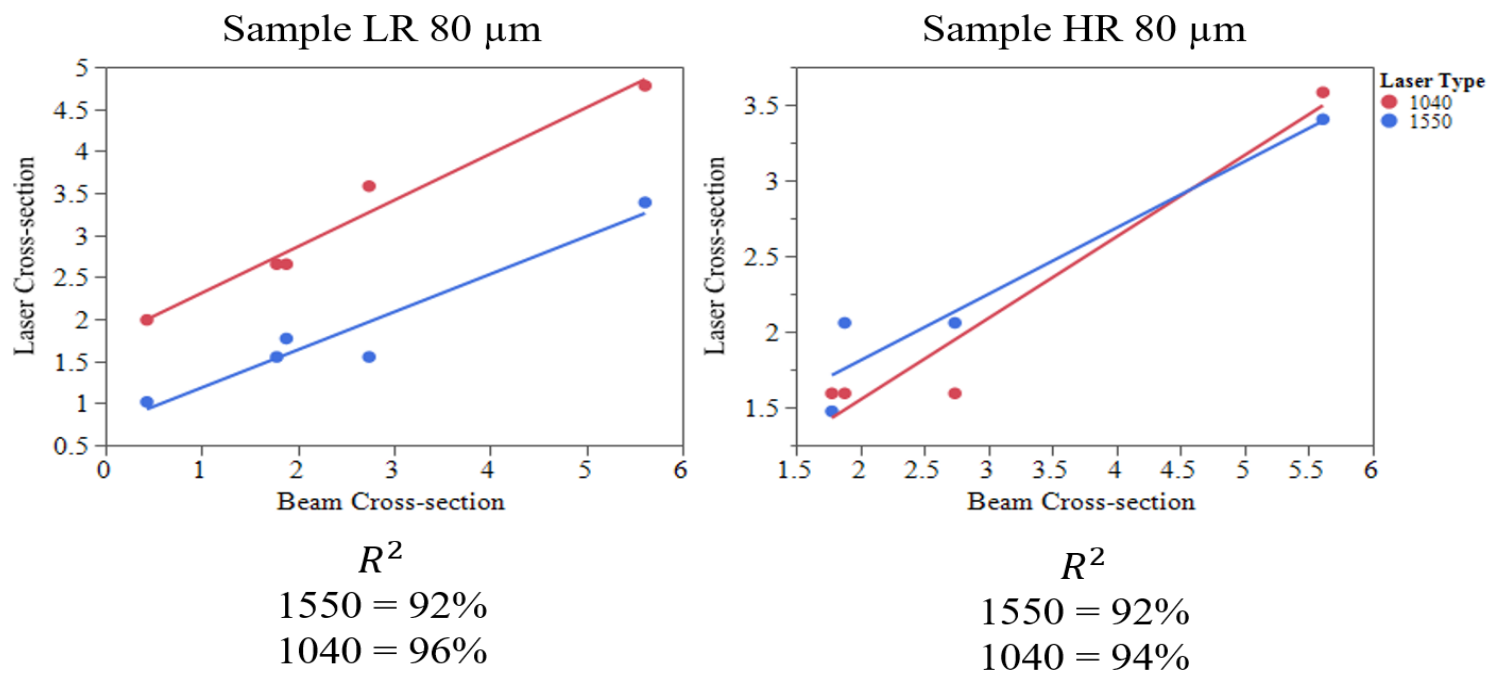

Figure 7.13 - SPA and TPA vs. beam cross-section

\subsubsection{Total Number of Flips of $\mathrm{N}$ and P-type Devices}

Another unresolved effect detected in our experiments is shown in figure 7.14. We separated our data by $0 \rightarrow 1$ flips and $1 \rightarrow 0$ flips. $0 \rightarrow 1$ flips belong to devices that originally had stored 0 , and therefore are PMOS devices, and hence $1 \rightarrow 0$ refers to a NMOS device. In the $1040 \mathrm{~nm}$ laser results we observed what we predicted. The curve of PMOS devices is ahead of NMOS, because as we have shown PMOS devices are weaker and have lower threshold energies. We expected to see a similar result with less defined boundary between the NMOS and PMOS devices, because of carrier diffusion. However, we were only able to detect a very small number of NMOS devices that were flipped. We were not able to provide an explanation for this result. This gives us an opportunity for further research on this issue and other unknown effects. One thing to consider is related 
to the cold and hot carriers described in chapter 7 . We mentioned that the excess energy left after a TPA event will cause further diffusion. The "heat" left behind will be absorbed by electrons and holes. Is this energy divided between the electron and hole based on their effective mass? Answering such questions may help us shine some light on this topic.

Our experimental results of TPA was presented in this chapter. As expected the $1550 \mathrm{~nm}$ laser is capable of penetrating through the full wafer thickness by means of TPA. One of the interesting findings of this work was the much higher photocurrent detection in $\mathrm{HR}$ than LR, and yet getting lower number of bits flipped in HR samples. Acceptable correlation was found between $1550 \mathrm{~nm}$ laser beam and particle accelerator further confirming that laser can be a viable alternative to beam testing for SER.
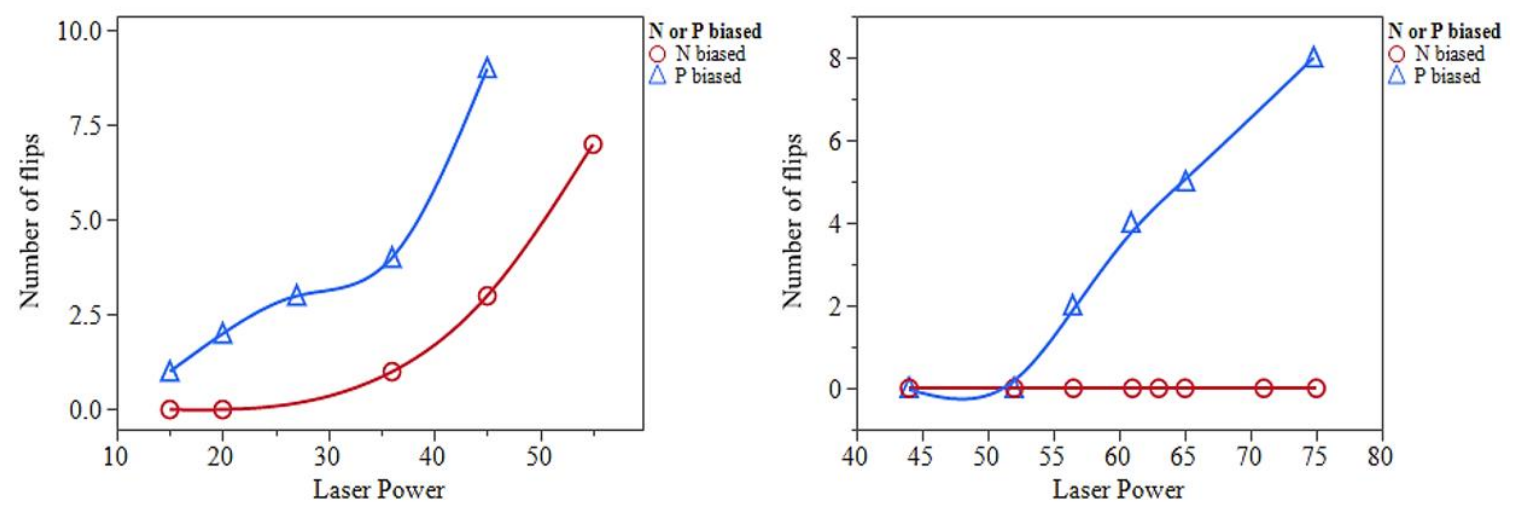

Figure 7.14 - Number of flips as a function of laser power for $1040 \mathrm{~nm}$ (left) and $1550 \mathrm{~nm}$ laser (right) 


\section{Chapter 8: Experiments and Results of Laser Testing}

In addition to direct study of SER, laser provides an attractive tool to study other effects in semiconductors. In this chapter, we will present a few of these findings such as temperature effect and the neighbor effect. Having access to in-house laser was a great advantage that led to design and performance of these tests.

\subsection{Bipolar amplification}

Several basic mechanisms affect charge transport and charge collection after an ion hits a circuit node. Bipolar amplification is the transient current leakage as a result of parasitic PNP junction formation. The total charge collected is the sum of funnel-aided drift, diffusion, and bipolar amplification.

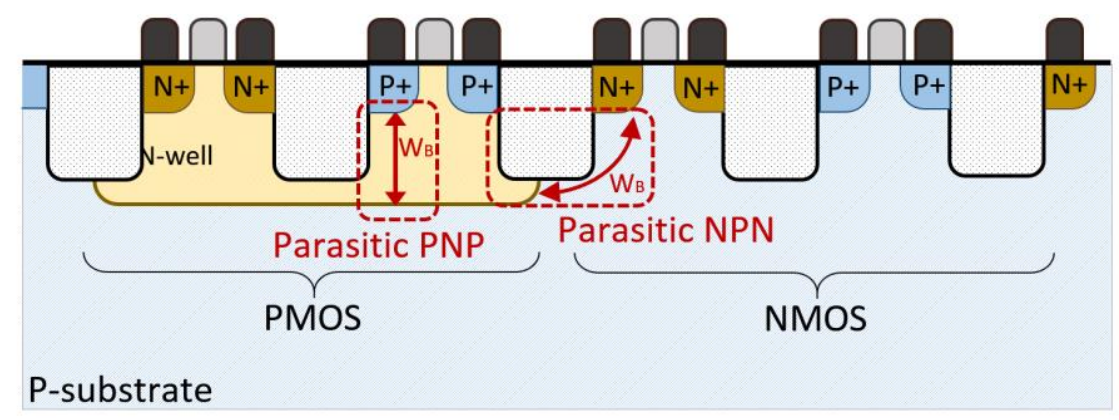

Figure 8.1 - Parasitic bipolar elements. The NMOS device has a lateral parasitic NPN bipolar transistor and the PMOS device has a lateral parasitic PNP bipolar transistor. (Oluwole A. Amusan, IEEE 2006 [21])

Parasitic bipolar junctions form within the CMOS devices as a result of close proximity of devices and reduced spacing between devices. Figure 8.1 shows the parasitic lateral and vertical BJTs formation within a CMOS device. The N+ source of the N-channel MOS device could act as the emitter of lateral NPN device; the P-substrate is the base and the Nwell is the collector of this device. P-substrate is the collector of a vertical PNP device, the 
$\mathrm{N}$-well acts as the base, and the $\mathrm{N}+$ source of the P-channel MOS device serve as the emitter of the PNP [34]. Following a particle strike at the drain/well junction, numerous electronhole pairs are generated along the ion track. Electrons can be collected quickly, but holes can only escape through the body contact (p-tap) or by recombination. Residual holes left in the well raise the well potential and lower the source/well potential barrier, causing the source to inject electrons into the channel. These electrons can be collected by the drain, where they can add to the original particle-induced current and cause an increased charge collection. This is the well-known parasitic bipolar amplification effect, where the source acts as the emitter, the bulk as the base region, and the drain as the collector. Injecting photocurrent in the well will reduce the well potential causing some charge to get pulled out of the source of the PMOS device which is connected to VCC. This is an ultrafast process.

\subsection{Temperature Dependence of SER}

NMOS and PMOS structures of an LR sample were targeted with laser energies initially low and incrementally increasing until the required energy to induce a flip (threshold energy) was found. The samples contain different size $\mathrm{N}$ and $\mathrm{P}$ type flip-flops as described in section 7.3. Supply voltage, VCC, was stepped at $0.1 \mathrm{~V}$ increments, starting from $0.6 \mathrm{~V}$ to $0.9 \mathrm{~V}$. We carried out the experiment at room temperature, 40C and 80C. Samples were thinned to 30 microns to minimize bulk absorption. 

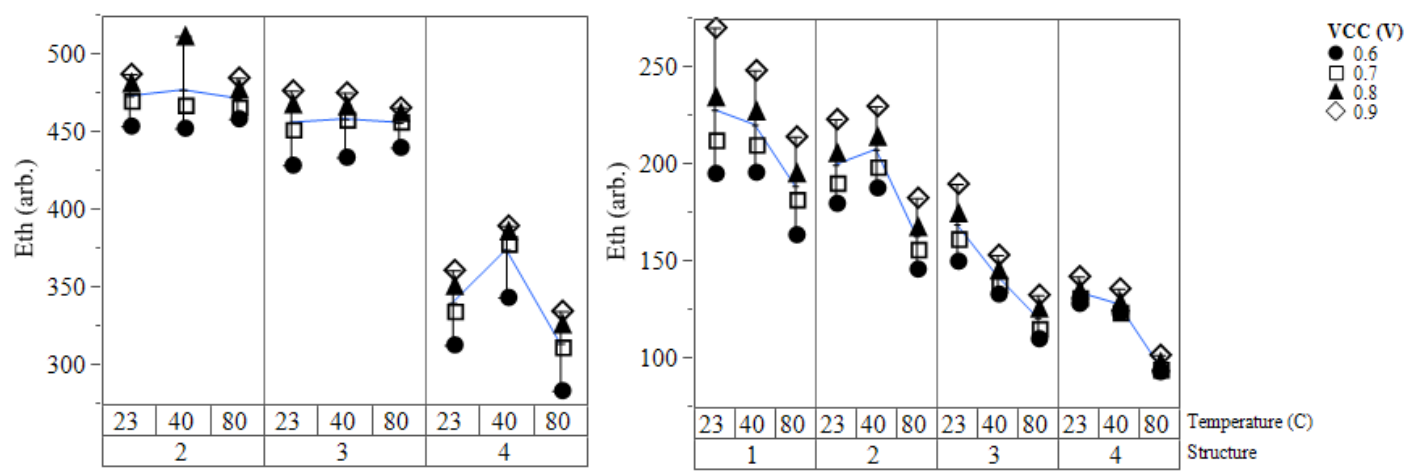

Figure 8.2 - Threshold energy of NMOS (left) and PMOS (right) latches at different temperatures

Comparing the threshold energies of NMOS and PMOS devices of figure 8.2 shows that threshold energies of PMOS devices are significantly smaller (about half) than those of NMOS devices of the same flavor, indicating the PMOS devices to be weaker and easier to upset. Additionally, threshold energy decreases with an increase in temperatures. This effect is more apparent in PMOS devices.

To further investigate the bipolar effect, we used discrete transistors on a testchip and targeted them with different $1040 \mathrm{~nm}$ laser at different powers. Figure 8.8 shows the test structures. Figure 8.3 is our measurements of bipolar amplification of PMOS and NMOS devices.

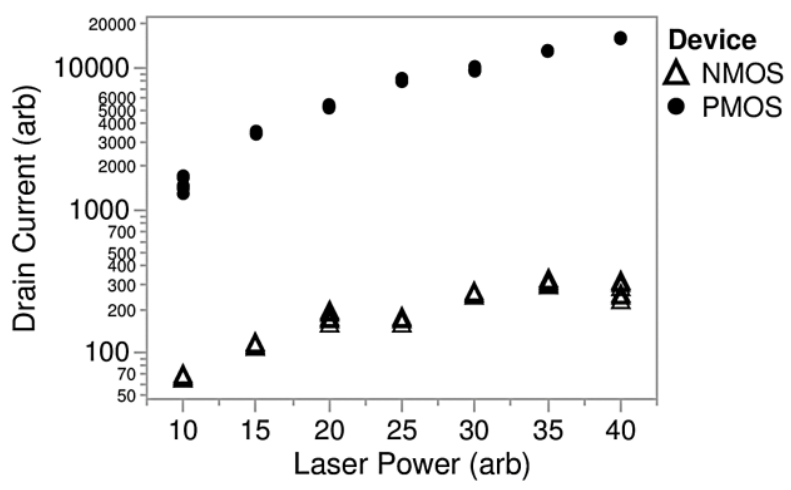

Figure 8.3 - NMOS and PMOS drain current as a function of Laser power 
PMOS devices experience a strong amplification as a result of bipolar effect caused by formation of lateral and vertical parasitic bipolar junctions described in 7.1. [36] We propose that the strong temperature dependency demonstrated in figure 8.2 is also a manifestation of parasitic BJTs. Moreover, the difference between the NMOS and PMOS threshold energies can be explained by considering the base width of these parasitic BJTs. The temperature dependence of bipolar transistors is a complex function of multitude of parameters including common-emitter current gain, $\beta$, which itself depends on both the emitter efficiency, $\gamma_{E}$, and base transport factor, $\alpha_{T}$.

$$
\beta=\frac{I_{C}}{I_{B}}=\frac{\alpha}{1-\alpha}
$$

where $\alpha$ is the common-base current gain. $\alpha$ itself can be written as

$$
\alpha=\alpha_{T} \gamma_{E} \delta_{r}
$$

where $\alpha_{T}$ is the base-transport factor, $\gamma_{E}$ is the emitter efficiency, and $\delta_{r}$ is the depletion layer recombination factor. Base transport factor is the efficiency of minority carrier transport through the base:

$$
\alpha_{T}=1-\frac{w_{B}{ }^{2}}{2 D_{B} \tau_{B}}
$$

where $w_{B}$ is the base width, $D_{B}$ is the carrier diffusion length in base, and $\tau_{B}$ is the recombination lifetime. Even though $\beta$ is not directly a function of temperature, $D_{B}$ has a temperature dependence:

$$
D_{B}=\mu k_{B} T
$$


where $\mu$ is the carrier mobility, and $k_{B}$ is Boltzmann constant. Here we can see that gain of the parasitic junction is inversely proportional to the squared of the base width and it is directly proportional to temperature:

And

$$
\beta \propto \frac{1}{w_{B}^{2}}
$$

$$
\beta \propto T
$$

For the vertical PNP device, the base width is the distance from the source $\mathrm{P}+$ to the bottom of the $\mathrm{N}$-well. This in general is smaller than the distance between source $\mathrm{N}+$ and the $\mathrm{N}$ well (figure 8.1). The base width of the parasitic NPN is generally much larger than that of the parasitic PNP, making the NPN parasitic BJT an inefficient bipolar amplifier as it can be seen from our results (figure 8.2). The bipolar gain of both parasitic PNP and NPN is dependent on temperature. However, this effect is a significantly more obvious in PNP than NPN. More precise data collection may be needed to detect this effect in NPN.

To validate the bipolar amplification model, PNP transistors where targeted with different laser powers at different temperatures (Figure 8.4). Current amplification was observed on PMOS transistors. No such amplification was found in NMOS. This amplification is due to existence of the $\mathrm{N}$-well and the formation of parasitic bipolar transistors. Note that there is a noticeable jump from $60 C$ to $80 C$. This effect was not observed in electrical measurements discussed in the next section. 


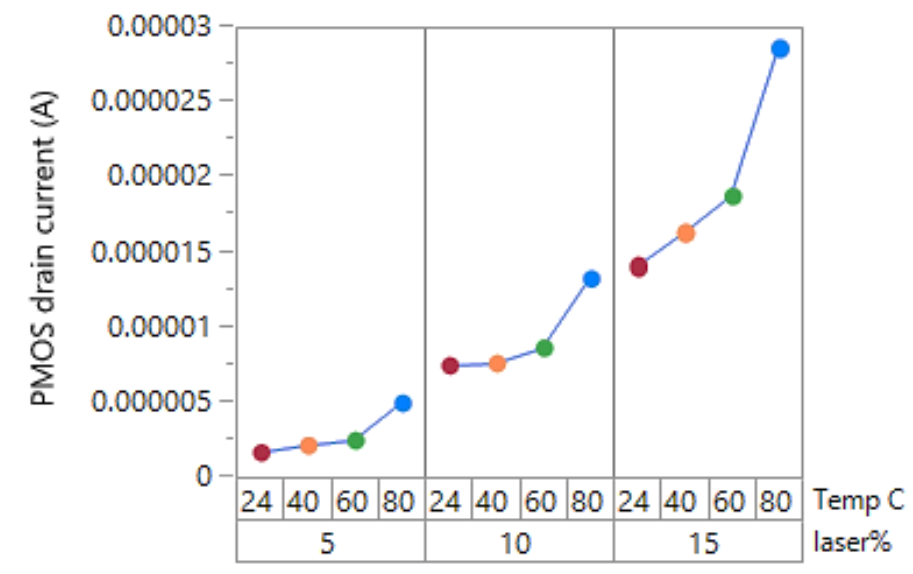

Figure 8.4 - Parasitic bipolar effect in a PNP structure - Laser testing

\subsection{Gain measurements}

We attempted to extract the parasitic junction gain electrically by sweeping the base of a PNP device and measuring $I_{\text {collector }}$ and $I_{\text {base }}$.

$$
\text { Gain }=\frac{I_{\text {collector }}}{I_{\text {base }}}
$$

We were not able to observe the same gain increase from $60 C$ to $80 C$ as in laser testing. We believe this is due to the fact that electrically we only inject majority carriers into the device, while optically we inject electron-hole pairs into the device which produces both majority and minority carriers. Additionally, in ultrafast laser experiment, charge transfer is an instantaneous event while the electrical testing is pseudo DC and charge injection is a gradual process, and therefore it causes saturation and that can be the reason we cannot observe the same effect. This highlights the importance of laser injection over classic methods like electrical 


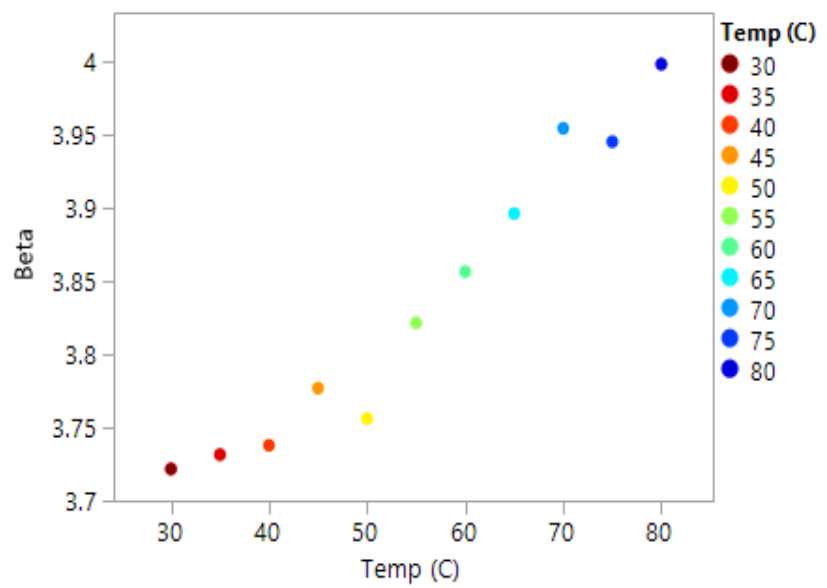

Figure 8.5 - Gain at different temperatures of PNP - Electrical testing

\subsection{Neighbor Effect}

The "neighbor effect" phenomenon is another consequence of close proximity of devices in modern CMOS technologies. It is a result of parasitic BJTs forming within the CMOS devices. A CMOS device is composed of complementary and symmetrical pairs of p-type and n-type MOSFETs. The $\mathrm{N}$ of a CMOS device is manufactured on a P-type substrate while the $\mathrm{P}$ is manufactured in an $\mathrm{N}$-type well within the $\mathrm{P}$ substrate (figure 8.6).

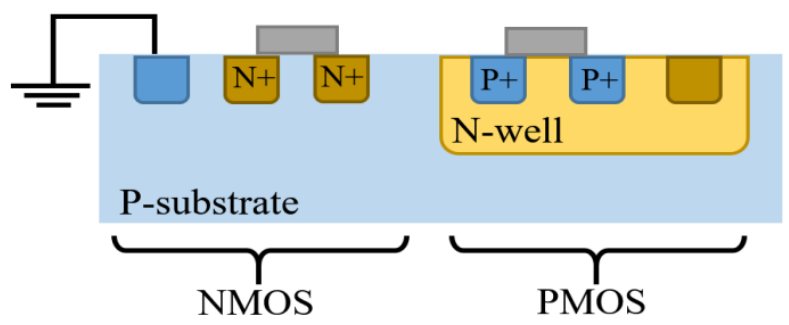

Figure 8.6 - A cartoon of CMOS device

A P-type substrate tap is connected to VSS and an N-type n-well tap is connected to VCC. Previously in section 8.2 we observed the effect of laser injection on the OFF device in a 
latch and the consequences of formation of parasitic bipolar amplification. Here we considered the same effect of reduction of the well potential but this time we observed its effect on the ON device: The neighbor effect (figure 8.7). Laser was injected at discrete

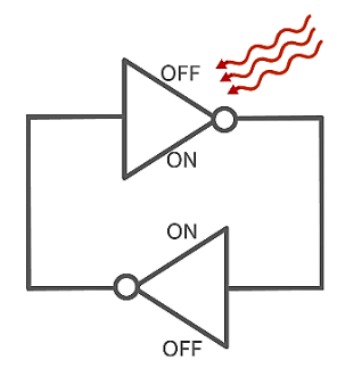

Figure 8.7 - ON and OFF device in a latch

PMOS and NMOS devices in normal condition on a testchip, and the drain current of the device in $\mathrm{ON}$ condition was measured immediately after laser injection, and without laser. We collected data at various temperature conditions. Figure 8.8 is a diagram of experiment showing each device in ON and OFF condition while targeted with the laser. The dark drain current (absence of laser) was also measured in each case. This dark current was taken into account in our results.
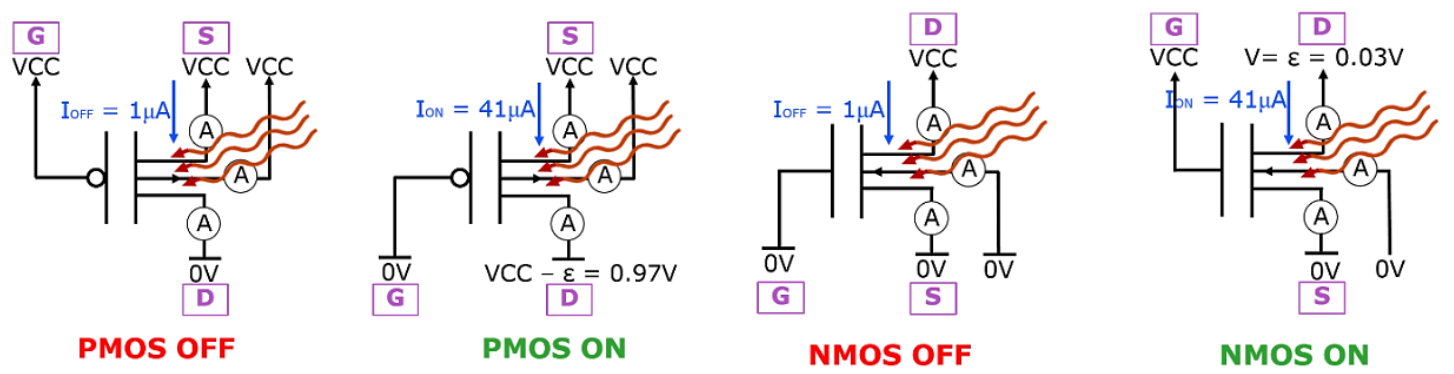

Figure 8.8 - PMOS devices in ON and OFF configurations with laser on

It can be seen from our results (figure 8.9) that while the NMOS device current gains about $40 \%$ the PMOS device current has dropped by about $80 \%$. This effect can be hypothesized 
as follows: injecting laser in the well area under device 1 (figure 8.10) will bias the base of the parasitic bipolar junction in the PMOS leading to bipolar amplification. Figure (8.10) demonstrates a top view of the parasitic PNP formed within the device.

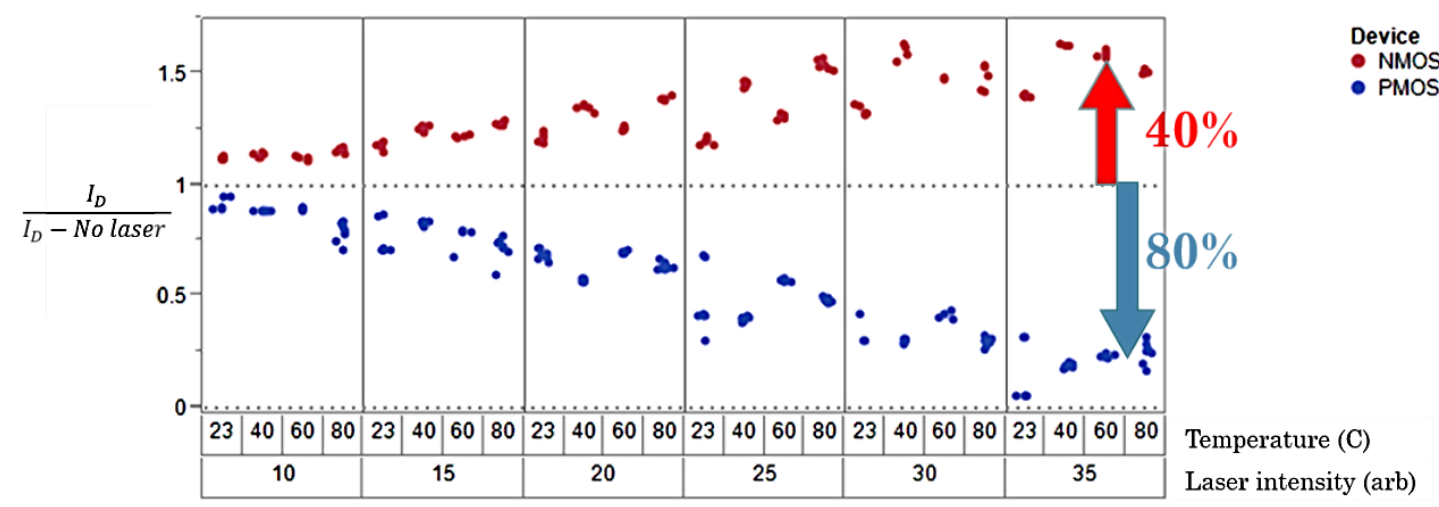

Figure 8.9 - Drain current for NMOS and PMOS devices on the ON configuration. 1 means no laser injection

This causes a momentarily decrease in potential of that local area due to self-rectification of well-substrate pn junction. This potential decrease will pull some of the charge out of device 2, causing the $\mathrm{ON}$ device to be slightly less $\mathrm{ON}$. This effect is due to relatively large distance between the device and the nearest $\mathrm{N}_{\text {tap }}$, and it is less significant in NMOS devices because charges in PMOS are confined to the well while charges in NMOS are not.
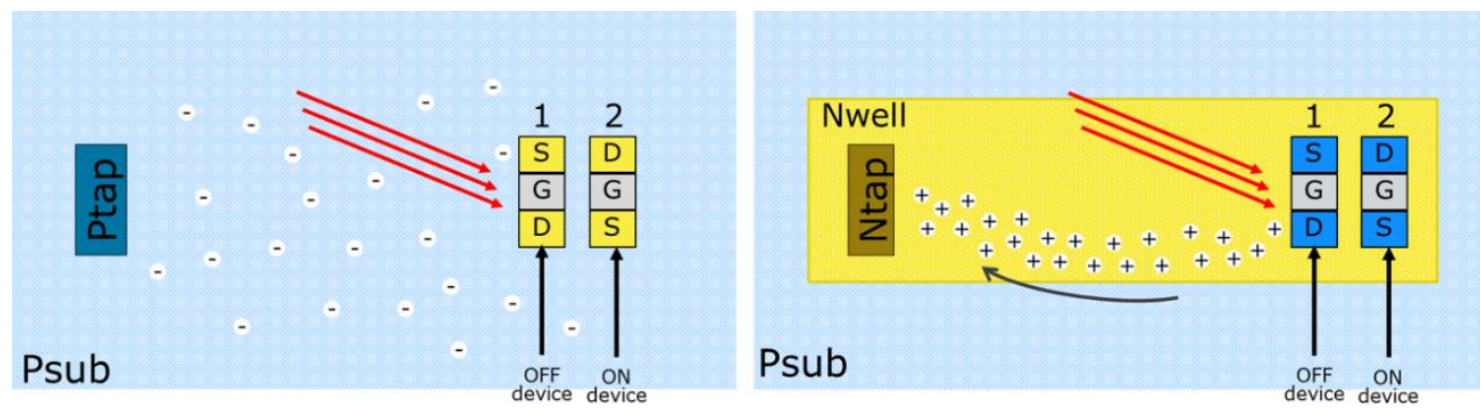

Figure 8.10 - Carrier path for NMOS (left) and PMOS (right) devices 
In the hypothetical situation where we could have a PMOS device with an "infinitely" large (relatively) $\mathrm{N}$-substrate, we would not observe the neighbor effect. Instead, we would detect the same amount of current for both $\mathrm{N}$ and $\mathrm{P}$ device. This "weakening mode" has not been reported before to our knowledge. 


\section{Chapter 9: Conclusion}

The work described in this disseration demonstrates that laser probing is a suitable tool for the study of radiation effects in CMOS devices and indeed can be used to reproduce faults and other phenomena on demand which would be very hard to study with a particle beam alone. The Neighbor Effect disscussed in chapter 8 is one example of such phenomena which is only possible to observe using laser probing. Another example is temperature dependance of SER covered in chapter 8, which can be explained by considering the base width of parasitic bipolar PNP junction formed within the PMOS device. In the course of the work presented here we also encountered some new and counterintuitive effects. For example, HR samples, though they register a higher photocurrent than LR samples, produce a lower number of flips due to a defocusing event described in chapter 7 . Additionally we obsereved that the high intensity $1550 \mathrm{~nm}$ laser tool is capable of producing comparable SER cross-section to the particle beam cross-section, and has the advantage of not requiring sample thinning, as is needed with a 1040 laser tool. The correlation results of section 7.3.2 can be improved by replacing the manual scanning method described at the beginning of section 7.3 with an automatic scaning process which will lead to a more consistent laser exposure of the sample and better overall results. The added insight into SER phenomena along with the flexible and relatively low cost nature of laser probing makes it a powerful tool for studying the interaction between ambient radiation and CMOS devices which plays out billions of times a day in deployed microelectronics around the world. 


\section{References}

1. Al Geist, "Monster in the Closet”, Spectrum IEEE, March 2016, p. 31-35

2. R. Gaillard, "Single Event Effects: Mechanisms and Classification”, (Springer 2011) - Soft Errors in Modern Electronic Systems edited by Michael Nicolaidis - chapter 2 Springer Science \& Business Media, Sep 24, 2010

3. J. F. Ziegler, "IBM Experiments in Soft Fails in Computer Electronics", IBM RES. DEVELOP. VOL.40 NO. 1, (January 1996)

4. Granlund, T., Olsson, N., "SEUs Induced by Thermal to High-Energy Neutrons in SRAMs”, IEEE Trans. Nuclear Science VOL. 53, NO. 6, (2006)

5. R.C. Baumann, "Radiation-Induced Soft Errors in Advanced Semiconductor Technologies”, IEEE Trans. Device and Materials Reliability, VOL. 5, NO. 3, (2005)

6. IBM, "Understanding Static RAM Operation” Article (1997)

7. M. Bohr, K. Mistry, “Intel's Revolutionary 22 nm Transistor Technology”, (2011)

8. N. Seifert, "Soft Error Susceptibilities of $22 \mathrm{~nm}$ Tri-Gate Devices", IEEE Trans. Nuclear Science, VOL. 59, NO. 6, (2012)

9. P. E. Dodd, "Current and Future Challenges in Radiation Effects on CMOS Electronics”, IEEE Trans. Nuclear Science VOL. 57, NO. 4 (2010)

10. Deen, M. Jamal, and Basu, Prasanta Kumar. Silicon Photonics, edited by M. Jamal Deen, and Basu, Prasanta Kumar, Wiley, 2012.

11. S. C. Baker-Finch, "Near-infrared Free Carrie Absorption in Heavily Doped Silicon", Journal of Applied Physics 116, 063106 (2014) 
12. K. G. Svantesson, "Determination of the Interband and the Free Carrier Absorption Constants in Silicon at High-Level Photoinjection”, J. Phys. D: Appl. Phys., VOL. 12, (1979)

13. R. S. Caldwell, D.S. Gage, G. H. Hanson, "The Transient Behavior of Transistors Due to Ionized Radiation Pulses", IEEE Trans. Comm. (1963)

14. S. P. Buchner, D. Wilson, K. Kang, D. Gill, J. A. Mazer, "Laser Simulation of Single Event Upsets”, IEEE Trans. Nuclear Science, VOL. NS-34, NO. 6, (1987)

15. El-Mamouni, "Pulsed Laser-induced Transient Currents in Bulk and Silicon-oninsulator FinFETs", IEEE Xplore (2011)

16. D. Ingerly, "Low-k Interconnect Stack with Metal-Insulator-Metal Capacitors for 22nm High Volume Manufacturing”, IEEE (2012)

17. R. Ascazubi, G. Modran, B. Gill, N. Seifert, "Laser-Assisted SER Estimation in Advanced CMOS Technologies, IEEE, (2013)

18. SunEdison Semiconductor, "High Resistivity Wafers", (2016)

19. D. Schroder, R. N. Tomas, J. Swartz, “Free Carrier Absorption in Silicon”, IEEE Journal of Solid-State Circuits, VOL. SC-13, NO. 1, (1978)

20. PV Lighthouse Free Carrier Absorption Calculator - Online: ttps://www2.pvlighthouse.com.au/calculators

21. N. Seifert, "On the Radiation-Induced Soft Error Performance of Hardened Sequential Elements in Advanced Bulk CMOS Technologies", IEEE (2010)

22. Singh, S. C., H.B. Zeng, Chunlei Guo, and H. B. Zeng. Nanomaterials, edited by S. C. Singh, et al., John Wiley \& Sons, Incorporated, 2012 
23. A. M. Weiner, "Ultrafast Optics", (John Wiley and Sons, 2009)

24. R. W. Boyd, “Nonlinear Optics”, (Academic Press, 2003)

25. L.J. Lewis and D. Perez, "Theory and Simulation of Laser Ablation - from Basic Mechanisms to Applications", Springer (2010)

26. G. Agrawal, "Nonlinear Fiber Optics", (Academic Press, 1995)

27. Cumberbatch, E. (1970). "Self-focusing in Non-linear Optics". IMA Journal of Applied Mathematics. 6 (3): 250-62.

28. G. C. Modoran, "Intense Field Electron Excitation in Transparent Materials", Dissertation (2005)

29. J. S. Melinger, Critical Evaluation of the Pulsed Laser Method for Single Event Effects Testing and Fundamental Studies", IEEE Trans. Nuclear Science, VOL. 41, NO. 6, (1994)

30. M. S. Gordon, P. Goldhagen, K. P. Rodbell, "Measurement of the Flux and Energy Spectrum of Cosmic-Ray Induced Neutrons on the Ground", IEEE Trans. Nuclear Science, VOL. 51, NO. 6, (2004)

31. R. Feynman, "Lectures on Physics", VOL. 2, (1965)

32. M. S. Tyagi and R. Van Overstraeten“, Minority carrier recombination in heavilydoped silicon, Solid-State Electron”, VOL. 26, pp. 577 (1983)

33. Tiberius Brastaviceanu, "Photoionization of Water in Self-focusing Regime", Editions universitaires europeennes (May 12, 2011) 
34. ON Semiconductor. (1989). Understanding Latch-Up in Advanced CMOS Logic. ON Semiconductor. Gresham, OR. [Online]. Available:

https://www.fairchildsemi.com/application-notes/AN/AN-600.pdf

35. S. M. Sze, K. K. Ng, "Physics of Semiconductor Devices", $3^{\text {rd }}$ edition, (John Wiley and Sons, 2007)

36. B. Ajdari, R. Ascazubi, G. Balkaran, "Temperature Dependance of SER Performance in 14nm FinFET Technology", Accepted for publication, Proceedings of SELSE 2017 Conference (2017) 